\title{
SPIN COVERS OF MAXIMAL COMPACT SUBGROUPS OF KAC-MOODY GROUPS AND SPIN-EXTENDED WEYL GROUPS
}

\author{
DAVID GHATEI, MAX HORN, RALF KÖHL, AND SEBASTIAN WEISS
}

\begin{abstract}
Let $G$ be a split real Kac-Moody group of arbitrary type and let $K$ be its maximal compact subgroup, i.e. the subgroup of elements fixed by a Cartan-Chevalley involution of $G$. We construct nontrivial spin covers of $K$, thus confirming a conjecture by Damour and Hillmann [DH09]. For irreducible simply laced diagrams and for all spherical diagrams these spin covers are two-fold central extensions of $K$. For more complicated irreducible diagrams these spin covers are central extensions by a finite 2-group of possibly larger cardinality. Our construction is amalgam-theoretic and makes use of the generalized spin representations of maximal compact subalgebras of split real Kac-Moody algebras studied in HKL13. Our spin covers contain what we call spin-extended Weyl groups which admit a presentation by generators and relations obtained from the one for extended Weyl groups by relaxing the condition on the generators so that only their eighth powers are required to be trivial.
\end{abstract}

\section{INTRODUCTION}

In [DH09, Section 3.5] it turned out that the existence of a spin-extended Weyl group $W^{\operatorname{spin}}\left(E_{10}\right)$ would be very useful for the study of fermionic billards. Lacking a concrete mathematical model of that group $W^{\operatorname{spin}}\left(E_{10}\right)$, Damour and Hillmann in their article instead use images of $W^{\text {spin }}\left(E_{10}\right)$ afforded by various generalized spin representations as described in [DKN06], [DBHP06], which can be realized as matrix groups.

In [DH09, Section 3.5, footnote 18, p. 24], Damour and Hillmann conjecture that the spin-extended Weyl group $W^{\text {spin }}\left(E_{10}\right)$ can be constructed as a discrete subgroup of a double spin cover $\operatorname{Spin}\left(E_{10}\right)$ of the subgroup $K\left(E_{10}\right)$ of elements fixed by the Cartan-Chevalley involution of the split real Kac-Moody group of type $E_{10}$. The purpose of this article is to confirm this conjecture, and to generalize it to arbitrary diagrams resp. arbitrary generalized Cartan matrices

In the simply laced case our result is as follows:

Theorem A. Let $\Pi$ be an irreducible simply laced Dynkin diagram, i.e., a Dynkin diagram affording only single edges, let $I=\{1, \ldots, n\}$ be a set of labels of the vertices of $\Pi$, and let $K(\Pi)$ be the subgroup of elements fixed by the Cartan-Chevalley involution of the split real Kac-Moody group of type $\Pi$. For each $i \in I$ let $G_{i} \cong \operatorname{Spin}(2)$ and for each $i \neq j \in I$ let

$$
G_{i j} \cong \begin{cases}\operatorname{Spin}(3), & \text { if } i, j \text { form an edge of } \Pi, \\ (\operatorname{Spin}(2) \times \operatorname{Spin}(2)) /\langle(-1,-1)\rangle, & \text { if } i, j \text { do not form an edge of } \Pi .\end{cases}
$$

Moreover, for $i<j \in I$, let $\phi_{i j}^{i}: G_{i} \rightarrow G_{i j}$ be the standard embedding as "upper-left diagonal block" and $\phi_{i j}^{j}: G_{j} \rightarrow G_{i j}$ be the standard embedding as "lower-right diagonal block".

Then up to isomorphism there exists a uniquely determined group, denoted $\mathrm{Spin}(\Pi)$, whose multiplication table extends the partial multiplication provided by $\left(\bigsqcup_{i<j \in I} G_{i j}\right) / \sim$ where $\sim$ is the equivalence relation determined by $\phi_{i j}^{i}(x) \sim \phi_{i k}^{i}(x)$ for all $i \neq j, k \in I$ and $x \in G_{i}$.

Furthermore, there exists a canonical two-to-one central extension $\mathrm{Spin}(\Pi) \rightarrow K(\Pi)$.

The system $\left\{G_{i}, G_{i j}, \phi_{i j}^{i}\right\}$ is called an amalgam of groups, the pair consisting of the group $\operatorname{Spin}(\Pi)$ and the set of canonical embeddings $\tau_{i}: G_{i} \hookrightarrow \operatorname{Spin}(\Pi), \tau_{i j}: G_{i j} \hookrightarrow \operatorname{Spin}(\Pi)$ a universal enveloping group; the canonical embeddings are called enveloping homomorphisms. Formal definitions and background information concerning amalgams can be found in Section 3 . Since all $G_{i} \cong \operatorname{Spin}(2)$ are isomorphic to one another, it in fact suffices to fix one group $U \cong \operatorname{Spin}(2)$ instead with connecting homomorphisms $\phi_{i j}^{i}: U \rightarrow G_{i j}$.

The formalization of the concept of standard embedding as "upper-left/lower right diagonal block" can be found in Section [10. Note that, since the $G_{i}$ are only given up to isomorphism, these standard embeddings are only well-defined up to automorphism of $G_{i}$, which leads to some ambiguity. Since by HKM13] the group $K(\Pi)$ (and therefore each of its central extensions by a finite group) is a topological group, one may assume the $\phi_{i j}^{i}$ to be continuous, thus restricting oneself to the ambiguity stemming 
from the two continuous automorphisms of $\operatorname{Spin}(2)$, the identity and the inversion homomorphisms. This ambiguity is resolved in Section 10 .

Theorem $\mathrm{A}$ provides us with the means of characterizing $W^{\text {spin }}(\Pi)$.

Theorem B. Let $\Pi$ be an irreducible simply laced Dynkin diagram, let $I=\{1, \ldots, n\}$ be a set of labels of the vertices of $\Pi$, and for each $i \in I$ let

- $\tau_{i}: G_{i} \cong \operatorname{Spin}(2) \hookrightarrow \operatorname{Spin}(\Pi)$ be the canonical enveloping homomorphisms,

- $x_{i} \in G_{i}$ elements of order eight whose polar coordinates involve the angle $\frac{\pi}{4}$, and

- $r_{i}:=\tau_{i}\left(x_{i}\right)$.

Then $W^{\operatorname{spin}}(\Pi):=\left\langle r_{i} \mid i \in I\right\rangle$ satisfies the defining relations

$$
\begin{array}{rlrl}
r_{i}^{8} & =1, & \\
r_{i}^{-1} r_{j}^{2} r_{i} & =r_{j}^{2} r_{i}^{2 n(i, j)} & & \text { for } i \neq j \in I, \\
\underbrace{r_{i} r_{j} r_{i} \cdots}_{m_{i j} \text { factors }} & =\underbrace{r_{j} r_{i} r_{j} \cdots}_{m_{i j} \text { factors }} & & \text { for } i \neq j \in I,
\end{array}
$$

where

$$
m_{i j}=\left\{\begin{array}{ll}
3, & \text { if } i, j \text { form an edge, } \\
2, & \text { if } i, j \text { do not form an edge, }
\end{array} \text { and } n(i, j)= \begin{cases}1, & \text { if } i, j \text { form an edge, } \\
0, & \text { if } i, j \text { do not form an edge. }\end{cases}\right.
$$

To be a set of defining relations means that any product of the $r_{i}$ that in $W^{\operatorname{spin}}(\Pi)$ represents the identity can be written as a product of conjugates of ways of representing the identity via (R1), (R2), (R3).

Our results in fact can be extended to arbitrary diagrams as discussed in Sections 16, 17, and 18,

As a by-product of our proof of Theorem $\mathrm{A}$ we show in Section 19 that for non-spherical diagrams $\Pi$ the groups $\operatorname{Spin}(\Pi)$ and $K(\Pi)$ are never simple; instead they always admit a non-trivial compact Lie group as a quotient via the generalized spin representation described in HKL13. The generalized spin representations of $\operatorname{Spin}(\Pi)$ is continuous, so that the obtained normal subgroups are closed. Similar non-simplicity phenomena as abstract groups have been observed in CH14. Furthermore, we observe that for arbitrary simply laced diagrams the image of $W^{\text {spin }}$ under the generalized spin representation is finite, generalizing [DH09, Lemma 2, p. 49].

Sections 3 4 5, 6, 8 are introductory in nature; we revise the notions of amalgams, Cartan matrices and Dynkin diagrams and fix our notation for orthogonal and spin groups. Sections 9 and 10 deal with the classification theory of amalgams and, as a blueprint for Theorem $\mathrm{A}$ identify $\mathrm{SO}(n)$ and $\operatorname{Spin}(n)$ as universal enveloping groups of $\mathrm{SO}(2)-$, resp. Spin(2)-amalgams of type $A_{n-1}$. In Section 11 we prove Theorem A.

Sections 13, 14, 15 provide us with the necessary tools for generalizing our findings to arbitrary diagrams; they deal with equivariant coverings of the real projective plane by the split Cayley hexagon and the symplectic quadrangle and with coverings of the real projective plane and the symplectic quadrangle by trees. In Section 16 we study SO(2)- and Spin(2)-amalgams for this larger class of diagrams. Section 17 deals with the general version of Theorem $\mathrm{A}$ Section 18 deals with the proof of Theorem $\mathrm{B}$ and its generalization. In Section 19 we observe that our findings provide epimorphisms from $\operatorname{Spin}(\Pi)$ and $K(\Pi)$ onto non-trivial compact Lie groups.

Acknowledgements. We thank Thibault Damour for pointing out his conjecture to us and Arjeh Cohen for very many valuable discussions concerning maximal compact subgroups of split real Lie groups of rank two. We also thank Guntram Hainke and Paul Levy for their comments and ideas concerning spin covers and Pierre-Emmanuel Caprace for several comments on a preliminary version of this article. The third named author moreover gratefully acknowledges the hospitality of the IHES in Bures-sur-Yvette and the Albert Einstein Institute in Golm. This research has been partially funded by the EPRSC grant $\mathrm{EP} / \mathrm{H} 02283 \mathrm{X}$.

\section{Contents}

1. Introduction

Part I. Basics

2. Conventions 
3. Amalgams

4. Cartan matrices and Dynkin diagrams

5. The groups $\mathrm{SO}(n)$ and $\mathrm{O}(n)$

6. The groups $\operatorname{Spin}(n)$ and $\operatorname{Pin}(n)$

7. The isomorphism $\operatorname{Spin}(4) \cong \operatorname{Spin}(3) \times \operatorname{Spin}(3)$

8. Lifting automorphism from $\mathrm{SO}(n)$ to $\operatorname{Spin}(n)$

Part II. Simply laced diagrams

9. $\mathrm{SO}(2)$-amalgams of simply laced type

10. Spin(2)-amalgams of simply laced type

11. Spin covers of simply laced type

Part III. Non-simply laced rank two diagrams

12. Strategies for reducing the general case to the simply-laced one $\quad 27$

\begin{tabular}{ll} 
13. Diagrams of type $\mathrm{G}_{2}$ & 28 \\
\hline
\end{tabular}

14. Diagrams of type $\mathrm{C}_{2} \quad 32$

15. Non-spherical diagrams of rank two

$\begin{array}{llr}\text { Part IV. Arbitrary diagrams } & 40\end{array}$

16. Admissible colourings $\quad 40$

17. Spin covers of arbitrary type 45

Part V. Applications $\quad 49$

18. Spin-extended Weyl groups $\quad 49$

19. Finite-dimensional compact Lie group quotients of Spin(П) and $K(\Pi)$

References

Part I. Basics

\section{Conventions}

Notation 2.1. $\mathbb{N}:=\{1,2,3, \ldots\}$ denotes the set of positive integers.

Notation 2.2. Throughout this article we use the convention $i j:=\{i, j\}$ if the set $\{i, j\}$ is used as an index. For example, if $G_{i j}$ is a group, then $G_{j i}$ is the same group. Note that this does not apply to superscripts, so $G^{i j}$ and $G^{j i}$ may differ.

Notation 2.3. For any group $G$, consider the following maps:

$$
\begin{array}{cc}
\text { inv }: G \rightarrow G: x \mapsto x^{-1}, & \text { the inverse map, } \\
\text { sq }: G \rightarrow G: x \mapsto x^{2}, & \text { the square map. }
\end{array}
$$

Both maps commute with any group homomorphism.

Notation 2.4. For any group $G$, we denote by $Z(G)$ the centre of $G$.

\section{Amalgams}

In this section we recall the concept of amalgams. More details concerning this concept can, in various formulations, be found in [BH99, Part III.C], [IS02, Section 1.3], GGH10, Section 1].

Definition 3.1. Let $U$ be a group, and $I \neq \emptyset$ a set. A $\boldsymbol{U}$-amalgam over $\boldsymbol{I}$ is a set

$$
\mathcal{A}=\left\{G_{i j}, \phi_{i j}^{i} \mid i \neq j \in I\right\}
$$

such that $G_{i j}$ is a group and $\phi_{i j}^{i}: U \rightarrow G_{i j}$ is a monomorphism for all $i \neq j \in I$. The maps $\phi_{i j}^{i}$ are called connecting homomorphisms. The amalgam is continuous if $U$ and $G_{i j}$ are topological groups, and $\phi_{i j}^{i}$ is continuous for all $i \neq j \in I$.

Definition 3.2. Let $\widetilde{\mathcal{A}}=\left\{\widetilde{G}_{i j}, \widetilde{\phi}_{i j}^{i} \mid i \neq j \in I\right\}$ and $\mathcal{A}=\left\{G_{i j}, \phi_{i j}^{i} \mid i \neq j \in I\right\}$ be $U$-amalgams over $I$. An epimorphism, resp. isomorphism $\alpha: \widetilde{\mathcal{A}} \rightarrow \mathcal{A}$ of $U$-amalgams is a system

$$
\alpha=\left\{\pi, \alpha_{i j} \mid i \neq j \in I\right\}
$$


consisting of a permutation $\pi \in \operatorname{Sym}(I)$ and group epimorphisms, resp. isomorphisms

$$
\alpha_{i j}: \widetilde{G}_{i j} \rightarrow G_{\pi(i) \pi(j)}
$$

such that for all $i \neq j \in I$

$$
\alpha_{i j} \circ \widetilde{\phi}_{i j}^{i}=\phi_{\pi(i) \pi(j)}^{\pi(i)},
$$

that is, the following diagram commutes:

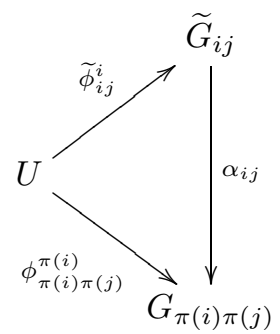

More generally, let $\widetilde{\mathcal{A}}=\left\{\widetilde{G}_{i j}, \widetilde{\phi}_{i j}^{i} \mid i \neq j \in I\right\}$ be a $U$-amalgam and let $\mathcal{A}=\left\{G_{i j}, \phi_{i j}^{i} \mid i \neq j \in I\right\}$ be a $V$-amalgam. An epimorphism $\alpha: \widetilde{\mathcal{A}} \rightarrow \mathcal{A}$ is a system

$$
\alpha=\left\{\pi, \rho^{i}, \alpha_{i j} \mid i \neq j \in I\right\}
$$

consisting of a permutation $\pi \in \operatorname{Sym}(I)$, group epimorphisms $\rho^{i}: U \rightarrow V$ and group epimorphisms

$$
\alpha_{i j}: \widetilde{G}_{i j} \rightarrow G_{\pi(i) \pi(j)}
$$

such that for all $i \neq j \in I$

$$
\alpha_{i j} \circ \widetilde{\phi}_{i j}^{i}=\phi_{\pi(i) \pi(j)}^{\pi(i)} \circ \rho^{\pi(i)},
$$

that is, the following diagram commutes:

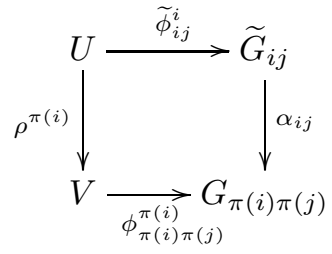

Notation 3.3. If (and only if) in the epimorphism $\alpha: \widetilde{\mathcal{A}} \rightarrow \mathcal{A}$ each $\rho^{i}: U \rightarrow V$ is an isomorphism, then one obtains an epimorphism $\alpha^{\prime}: \widetilde{\mathcal{A}} \rightarrow \mathcal{A}^{\prime}$ of $U$-amalgams by defining $\alpha^{\prime}=\left\{\pi, \alpha_{i j} \mid i \neq j \in I\right\}$ and $\mathcal{A}^{\prime}=\left\{G_{i j},\left(\phi_{i j}^{i}\right)^{\prime}\right\}$ via

$$
\left(\phi_{i j}^{i}\right)^{\prime}: U \rightarrow G_{i j}: u \mapsto\left(\phi_{i j}^{i} \circ \rho^{i}\right)(u) .
$$

If this $\alpha^{\prime}$ turns out to be an isomorphism of $U$-amalgams, by slight abuse of terminology we also call the epimorphism $\alpha$ an isomorphism of amalgams.

Remark 3.4. More generally, an amalgam can be defined as a collection of groups $G_{i}$ and a collection of groups $G_{i j}$ with connecting homomorphisms $\psi_{i j}^{i}: G_{i} \rightarrow G_{i j}$. Since in our situation for all $i$ there exist isomorphisms $\gamma_{i}: U \rightarrow G_{i}$, it suffices to consider the connecting homomorphisms $\phi_{i j}^{i}=\psi_{i j}^{i} \circ \gamma_{i}$.

In the more general setting, an isomorphism of amalgams consists of a permutation $\pi$ of the index set $I$ and isomorphisms $\alpha_{i}: G_{i} \rightarrow \bar{G}_{\pi(i)}$ and $\alpha_{i j}: G_{i j} \rightarrow \bar{G}_{\pi(i) \pi(j)}$ such that

$$
\alpha_{i j} \circ \psi_{i j}^{i}=\bar{\psi}_{\pi(i) \pi(j)}^{\pi(i)} \circ \alpha_{i} .
$$

A routine calculation shows that $U$-amalgams and isomorphisms of $U$-amalgams are special cases of amalgams and isomorphisms of amalgams as found in the literature.

Definition 3.5. Given a $U$-amalgam $\mathcal{A}=\left\{G_{i j}, \phi_{i j}^{i} \mid i \neq j \in I\right\}$, an enveloping group of $\mathcal{A}$ is a pair $(G, \tau)$ consisting of a group $G$ and a set

$$
\tau=\left\{\tau_{i j} \mid i \neq j \in I\right\}
$$

of enveloping homomorphisms $\tau_{i j}: G_{i j} \rightarrow G$ such that

$$
G=\left\langle\tau_{i j}\left(G_{i j}\right) \mid i \neq j \in I\right\rangle, \quad \forall i \neq j \neq k \in I: \tau_{i j} \circ \phi_{i j}^{j}=\tau_{k j} \circ \phi_{k j}^{j},
$$


that is, for $i \neq j \neq k \in I$ the following diagram commutes:

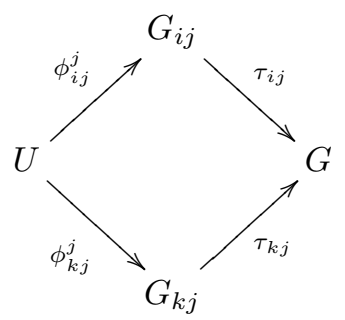

We write $\tau: \mathcal{A} \rightarrow G$ and call $\tau$ an enveloping morphism. An enveloping group $(G, \tau)$ and the corresponding enveloping morphism are faithful if $\tau_{i j}$ is a monomorphism for all $i \neq j \in I$.

Definition 3.6. Given a $U$-amalgam $\mathcal{A}=\left\{G_{i j}, \phi_{i j}^{i}\right\}$, an enveloping group $(G, \tau)$ is called a universal enveloping group if, given an enveloping group $\left(H, \tau^{\prime}\right)$ of $\mathcal{A}$, there is a unique epimorphism $\pi: G \rightarrow H$ such that for all $i \neq j \in I$ one has $\pi \circ \tau_{i j}=\tau_{i j}^{\prime}$. We write $\tau: \mathcal{A} \rightarrow G$ and call $\tau$ a universal enveloping morphism. By universality, two universal enveloping groups $\left(G_{1}, \tau_{1}\right)$ and $\left(G_{2}, \tau_{2}\right)$ of a $U$-amalgam $\mathcal{A}$ are (uniquely) isomorphic.

The canonical universal enveloping group of the $U$-amalgam $\mathcal{A}$ is the pair $(G(\mathcal{A}), \widehat{\tau})$, where $G(\mathcal{A})$ is the group given by the presentation

$$
\left.G(\mathcal{A}):=\left\langle\bigcup_{i \neq j \in I} G_{i j}\right| \text { all relations in } G_{i j}, \text { and } \forall i \neq j \neq k \in I, \forall x \in U: \phi_{i j}^{j}(x)=\phi_{k j}^{j}(x)\right\rangle
$$

and where $\widehat{\tau}=\left\{\widehat{\tau}_{i j} \mid i \neq j \in I\right\}$ with the canonical homomorphism $\widehat{\tau}_{i j}: G_{i j} \rightarrow G(\mathcal{A})$ for all $i \neq j \in I$. The canonical universal enveloping group of a $U$-amalgam is a universal enveloping group (cf. IS02, Lemma 1.3.2]).

Lemma 3.7. Let $U$ and $V$ be groups and $I$ an index set. Suppose

- $\widetilde{\mathcal{A}}=\left\{\widetilde{G}_{i j}, \widetilde{\phi}_{i j}^{i} \mid i \neq j \in I\right\}$ is a $U$-amalgam over $I$,

- $\mathcal{A}=\left\{G_{i j}, \phi_{i j}^{i} \mid i \neq j \in I\right\}$ is a $V$-amalgam over $I$,

- $\alpha=\left\{\pi, \rho^{i}, \alpha_{i j} \mid i \neq j \in I\right\}$ is an amalgam epimorphism $\widetilde{\mathcal{A}} \rightarrow \mathcal{A}$,

- $(G, \tau)$ with $\left.\tau=\left\{\tau_{i j} \mid i \neq j \in I\right\}\right)$ is an enveloping group of $\mathcal{A}$.

Then the following hold:

(a) There is a unique enveloping group $(G, \widetilde{\tau}), \widetilde{\tau}=\left\{\widetilde{\tau}_{i j} \mid i \neq j \in I\right\}$, of $\widetilde{\mathcal{A}}$ such that the following diagram commutes for all $i \neq j \in I$ :

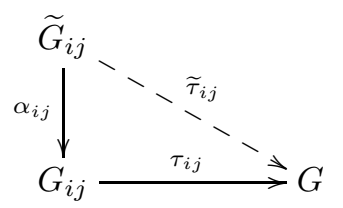

(b) Suppose $(\widetilde{G}, \widetilde{\tau}), \widetilde{\tau}=\left\{\widetilde{\tau}_{i j} \mid i \neq j \in I\right\}$, is a universal enveloping group of $\widetilde{\mathcal{A}}$. Then there is a unique epimorphism $\widehat{\alpha}: \widetilde{G} \rightarrow G$ such that the following diagram commutes for all $i \neq j \in I$ :

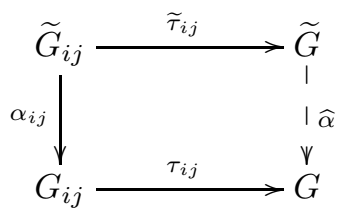

(c) If $\alpha$ is an isomorphism and $(G, \tau)$ is a universal enveloping group, then also $\widehat{\alpha}$ is an isomorphism.

Proof. $\quad$ (a) Let $i \neq j \in I$. Since $\alpha_{i j}$ is an epimorphism, we must have $\widetilde{\tau}_{i j}:=\tau_{i j} \circ \alpha_{i j}$ for the diagrams to commute; the claimed uniqueness follows. The fact that $\alpha_{i j}$ is an epimorphism also implies

$$
\widetilde{\tau}_{i j}\left(\widetilde{G}_{i j}\right)=\left(\tau_{i j} \circ \alpha_{i j}\right)\left(\widetilde{G}_{i j}\right)=\tau_{i j}\left(G_{i j}\right), \quad \text { and so } \quad G=\left\langle\tau_{i j}\left(\widetilde{G}_{i j}\right)\right\rangle=\left\langle\widetilde{\tau}_{i j}\left(\widetilde{G}_{i j}\right)\right\rangle . .
$$

Moreover, for $i \neq j \neq k \in I$ we find

$$
\begin{aligned}
\widetilde{\tau}_{i j} \circ \widetilde{\phi}_{i j}^{j}=\tau_{i j} \circ \alpha_{i j} \circ \widetilde{\phi}_{i j}^{j} & =\tau_{i j} \circ \phi_{\pi(i) \pi(j)}^{\pi(j)} \circ \rho^{\pi(j)} \\
& =\tau_{k j} \circ \phi_{\pi(k) \pi(j)}^{\pi(j)} \circ \rho^{\pi(j)}=\tau_{k j} \circ \alpha_{k j} \circ \widetilde{\phi}_{k j}^{j}=\widetilde{\tau}_{k j} \circ \widetilde{\phi}_{k j}^{j} .
\end{aligned}
$$


Hence $\left(G,\left\{\widetilde{\tau}_{i j}\right\}\right)$ is indeed an enveloping group of $\widetilde{\mathcal{A}}$.

(b) On the one hand, by (a) the lower left triangle in the following diagram commutes:

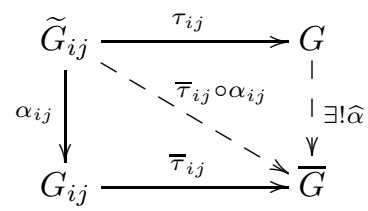

On the other hand, by the definition of universal enveloping group there is a unique epimorphism $\widehat{\alpha}$ making the upper right triangle commute. The claim follows.

(c) follows from (b) by exchanging the roles of $G_{i j}, G$ and $\widetilde{G}_{i j}, \widetilde{G}$.

Notation 3.8. We denote the situation in Lemma 3.7 (a) by the commutative diagram

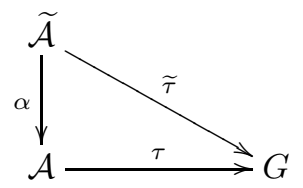

and the situation in Lemma 3.7 (b) by the commutative diagram

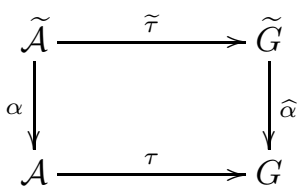

The following proposition will be crucial throughout this article. The typical situation in our applications will be $U=\operatorname{SO}(2), \widetilde{U}=\operatorname{Spin}(2), \widetilde{V}=\{ \pm 1\}$.

Proposition 3.9. Let $U, \widetilde{U}$ and $\widetilde{V} \leq \widetilde{U}$ be groups and $I$ an index set. Suppose

- $\widetilde{\mathcal{A}}=\left\{\widetilde{G}_{i j}, \widetilde{\phi}_{i j}^{i} \mid i \neq j \in I\right\}$ is a $\widetilde{U}$-amalgam over I such that $\widetilde{G}_{i j}=\left\langle\widetilde{\phi}_{i j}^{i}(\widetilde{U}), \widetilde{\phi}_{i j}^{j}(\widetilde{U})\right\rangle$,

- $\mathcal{A}=\left\{G_{i j}, \phi_{i j}^{i} \mid i \neq j \in I\right\}$ is a $U$-amalgam over $I$,

- $\alpha=\left\{\pi, \rho^{i}, \alpha_{i j} \mid i \neq j \in I\right\}$ is an amalgam epimorphism $\widetilde{\mathcal{A}} \rightarrow \mathcal{A}$,

- $(\widetilde{G}, \widetilde{\tau})$ with $\left.\widetilde{\tau}=\left\{\widetilde{\tau}_{i j} \mid i \neq j \in I\right\}\right)$ is a universal enveloping group of $\widetilde{\mathcal{A}}$,

- $(G, \tau)$ with $\left.\tau=\left\{\tau_{i j} \mid i \neq j \in I\right\}\right)$ is a universal enveloping group of $\mathcal{A}$,

- $\widehat{\alpha}: \widetilde{G} \rightarrow G$ is the epimorphism induced by $\alpha$ via the commutative diagrams $(i \neq j \in I)$

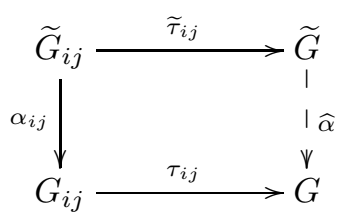

as in Lemma $3.7(b)$.

For $i \neq j \in I$ define $Z_{i j}^{i}:=\widetilde{\phi}_{i j}^{i}(\widetilde{V})$ and $Z_{i j}^{\widetilde{\phi}}:=\left\langle Z_{i j}^{i}, Z_{i j}^{j}\right\rangle$, as well as $A_{i j}:=\operatorname{ker}\left(\alpha_{i j}\right)$. Then if

$$
A_{i j} \leq Z_{i j}^{\widetilde{\phi}} \leq Z\left(\widetilde{G}_{i j}\right)
$$

it follows that $\widetilde{G}$ is a central extension of $G$ by $N:=\left\langle\widetilde{\tau}_{i j}\left(A_{i j}\right) \mid i \neq j \in I\right\rangle$.

In this situation the epimorphism $\alpha: \widetilde{\mathcal{A}} \rightarrow \mathcal{A}$ is called an $|N|$-fold central extension of amalgams.

Proof. We proceed by proving the following two assertions:

(a) $N \leq Z(\widetilde{G})$,

(b) $\widetilde{G} / N \cong G$. 
Consider the following commutative diagram:

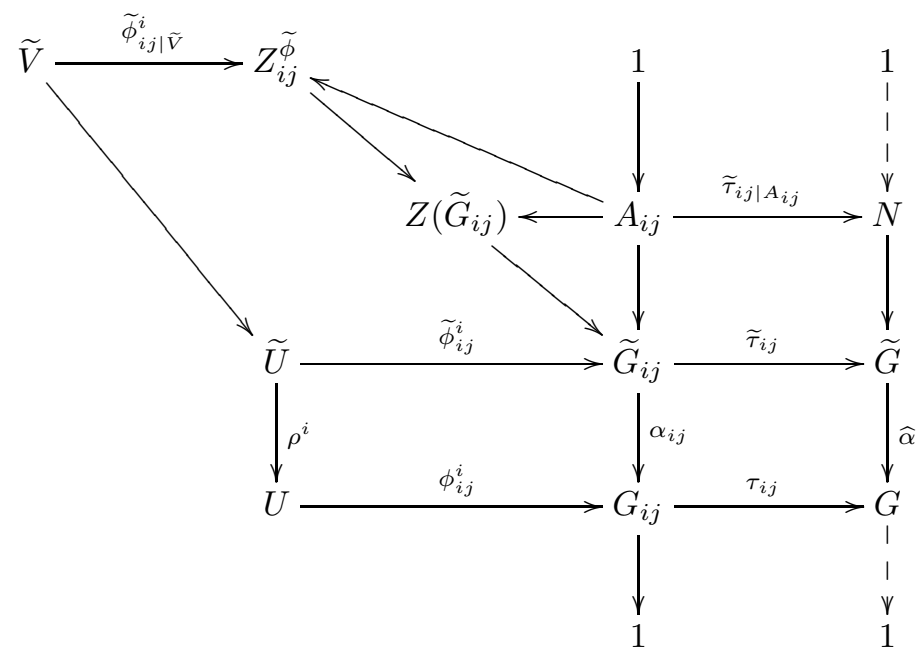

For $i \neq j \in I$ set

$$
Z_{i}:=\widetilde{\tau}_{i j}\left(Z_{i j}^{i}\right)=\left(\widetilde{\tau}_{i j} \circ \widetilde{\phi}_{i j}^{i}\right)(\widetilde{V}) \quad \text { and } \quad \widetilde{G}_{i}:=\left(\widetilde{\tau}_{i j} \circ \widetilde{\phi}_{i j}^{i}\right)(\widetilde{U}) .
$$

The hypothesis implies

$$
\widetilde{\tau}_{i j}\left(\widetilde{G}_{i j}\right)=\left\langle\widetilde{G}_{i}, \widetilde{G}_{j}\right\rangle \quad \text { and } \quad \widetilde{G}=\left\langle\widetilde{\tau}_{i j}\left(\widetilde{G}_{i j}\right) \mid i \neq j \in I\right\rangle=\left\langle\widetilde{G}_{i} \mid i \in I\right\rangle .
$$

Moreover,

$$
Z_{i}=\widetilde{\tau}_{i j}\left(Z_{i j}^{i}\right) \leq \widetilde{\tau}_{i j}\left(Z\left(\widetilde{G}_{i j}\right)\right) \leq Z\left(\widetilde{\tau}_{i j}\left(\widetilde{G}_{i j}\right)\right)=Z\left(\left\langle\widetilde{G}_{i}, \widetilde{G}_{j}\right\rangle\right)
$$

whence $Z_{i}$ centralizes $\widetilde{G}_{j}$ for all $i, j \in I$. Since $\widetilde{G}$ is generated by the $\widetilde{G}_{i}$, one has

$$
\left\langle Z_{i} \mid i \in I\right\rangle \leq Z\left(\left\langle\widetilde{G}_{i} \mid i \in I\right\rangle\right)=Z(\widetilde{G}) .
$$

Therefore

$$
N=\left\langle\widetilde{\tau}_{i j}\left(A_{i j}\right) \mid i \neq j \in I\right\rangle \leq\left\langle\widetilde{\tau}_{i j}\left(Z_{i j}^{i}\right) \mid i \neq j \in I\right\rangle=\left\langle Z_{i} \mid i \in I\right\rangle \leq Z(\widetilde{G}),
$$

i.e., (a) holds.

Commutativity of the diagram implies $N \leq \operatorname{ker}(\widehat{\alpha})$ and so the homomorphism theorem yields an epimorphism $\widetilde{G} / N \rightarrow G: g N \mapsto \widehat{\alpha}(g)$. In order to show that this epimorphism actually is an isomorphism we construct an inverse map by exploiting that $G$ and $\widetilde{G}$ are universal enveloping groups of $\mathcal{A}, \operatorname{resp}$. $\widetilde{\mathcal{A}}$. Indeed, for $g \in G_{i j}$, let $\widetilde{g} \in \alpha_{i j}^{-1}(g)$. Then

$$
\widetilde{\tau}_{i j}\left(\alpha_{i j}^{-1}(g)\right)=\widetilde{\tau}_{i j}\left(\widetilde{g} A_{i j}\right) \leq \widetilde{\tau}_{i j}(\widetilde{g}) N=\widetilde{\tau}_{i j}\left(\alpha_{i j}^{-1}(g)\right) N \in \widetilde{G} / N
$$

Thus we obtain a well-defined homomorphism

$$
\widehat{\tau}_{i j}: G_{i j} \rightarrow \widetilde{G} / N: g \mapsto \widetilde{\tau}_{i j}\left(\alpha_{i j}^{-1}(g)\right) N
$$

Then $\left(\widetilde{G} / N,\left\{\widehat{\tau}_{i j}\right\}\right)$ is an enveloping group for $\mathcal{A}$. In particular for $u \in U$ and $i \neq j \neq k \in I$ we have

$$
\begin{aligned}
\left(\widehat{\tau}_{i j} \circ \phi_{i j}^{j}\right)(u) & =\widetilde{\tau}_{i j}\left(\alpha_{i j}^{-1}\left(\phi_{i j}^{j}(u)\right)\right)=\widetilde{\tau}_{i j}\left(\widetilde{\phi}_{i j}^{j}\left(\left(\rho^{j}\right)^{-1}(u)\right)\right) \\
& =\widetilde{\tau}_{k j}\left(\widetilde{\phi}_{k j}^{j}\left(\left(\rho^{j}\right)^{-1}(u)\right)\right)=\widetilde{\tau}_{k j}\left(\alpha_{k j}^{-1}\left(\phi_{k j}^{j}(u)\right)\right)=\left(\widehat{\tau}_{k j} \circ \phi_{k j}^{j}\right)(u) .
\end{aligned}
$$

Since $\left(G,\left\{\tau_{i j}\right\}\right)$ is a universal enveloping group of $\mathcal{A}$, there exists a unique epimorphism $\beta: G \rightarrow \widetilde{G} / N$ such that for $i \neq j \in I$ we have

By the definition of $\widehat{\alpha}$ and $\widehat{\tau}_{i j}$ we find

$$
\beta \circ \tau_{i j}=\widehat{\tau}_{i j}
$$

$$
\widehat{\alpha} \circ \widehat{\tau}_{i j}=\tau_{i j}
$$

Therefore

$$
(\beta \circ \widehat{\alpha}) \circ \widehat{\tau}_{i j}=\widehat{\tau}_{i j} \quad \text { and } \quad(\widehat{\alpha} \circ \beta) \circ \tau_{i j}=\tau_{i j} .
$$

But $(G, \tau)$ and $(\widetilde{G}, \widetilde{\tau})$ are universal enveloping groups; their uniqueness property implies that $\beta \circ \widehat{\alpha}=\operatorname{id}_{\widetilde{G} / N}$ and $\widehat{\alpha} \circ \beta=\operatorname{id}_{G}$ and hence as claimed $\widetilde{G} / N \cong G$. We have shown assertion (b). 


\section{Cartan matrices and Dynkin diagrams}

In this section we recall the concepts of Cartan matrices and Dynkin diagrams. For a thorough introduction see [Kac90, Chapter 4], [Rém02, Section 7.1].

Definition 4.1. Let $I$ be a non-empty set. A generalized Cartan matrix over $I$ is a matrix $A=$ $(a(i, j))_{i, j \in I}$ such that for all $i \neq j \in I$,

(a) $a(i, i)=2$,

(b) $a(i, j)$ is a non-positive integer,

(c) if $a(i, j)=0$ then $a(j, i)=0$.

$A$ is of two-spherical type if $a(i, j) a(j, i) \in\{0,1,2,3\}$ for all $i \neq j \in I$.

Definition 4.2. A Dynkin diagram (or short: diagram) is a graph $\Pi$ with vertex set $V(\Pi)$ and edge set $E(\Pi) \subseteq\left(\begin{array}{c}V(\Pi) \\ 2\end{array}\right)$ such that each edge has an edge valency of $1,2,3$ or $\infty$ and, in addition, edges with valency 2 or 3 are directed. If $\{v, w\} \in E(\Pi)$ is directed from $v$ to $w$, we write $v \rightarrow w$. Let $E_{0}(\Pi):=\left(\begin{array}{c}V(\Pi) \\ 2\end{array}\right) \backslash E(\Pi)$, and let $E_{1}(\Pi), E_{2}(\Pi), E_{3}(\Pi)$, resp. $E_{\infty}(\Pi)$ be the subsets of $E(\Pi)$ of edges of valency $1,2,3$, resp. $\infty$. The elements of $E_{1}(\Pi), E_{2}(\Pi), E_{3}(\Pi)$ are called edges of type $A_{2}, \mathrm{C}_{2}$ resp. $\mathrm{G}_{2}$. The diagram $\Pi$ is irreducible if it is connected as a graph, it is simply laced if all edges have valency 1 , it is doubly laced if all edges have valency 1 or 2 , and it is two-spherical if no edge has valency $\infty$. If $V(\Pi)$ is finite, then a labelling of $\Pi$ is a bijection $\sigma: I \rightarrow V(\Pi)$, where $I:=\{1, \ldots,|V(\Pi)|\}$.

Throughout this text, we assume all diagrams to have finite vertex set.

Remark 4.3. Let $I$ be a non-empty set and $A=(a(i, j))_{i, j \in I}$ a two-spherical generalized Cartan matrix. Then this induces a two-spherical Dynkin diagram $\Pi(A)$ with vertex set $V:=I$ as follows: For $i \neq j \in I$, there is an edge between $i$ and $j$ if and only if $a(i, j) \neq 0$. The valency of the edge then is $q_{i j}:=$ $a(i, j) a(j, i) \in\{1,2,3\}$. If $q_{i j}>1$, then the edge is directed $i \leftarrow j$ if and only if $a(i, j)=-q<-1=a(j, i)$.

Conversely, given a two-spherical Dynkin diagram $\Pi$ with vertex set $V$, we obtain a two-spherical generalized Cartan matrix $A(\Pi):=(a(i, j))_{i, j \in I}$ over $I:=V$ by setting for $i \neq j \in I$ :

$$
a(i, i):=2, \quad a(i, j):= \begin{cases}0, & \text { if }\{i, j\} \notin E(\Pi), \\ -2, & \text { if }\{i, j\} \in E_{2}(\Pi) \text { and } i \leftarrow j, \\ -3, & \text { if }\{i, j\} \in E_{3}(\Pi) \text { and } i \leftarrow j, \\ -1, & \text { otherwise. }\end{cases}
$$

These two operations are inverse to each other, i.e., $\Pi(A(\Pi))=\Pi$ and $A(\Pi(A))=A$.

Notation 4.4. If the generalized Cartan matrix $A$ is not of two-spherical type, it is nevertheless possible to associate a Dynkin diagram $\Pi(A)$ to it by labelling the edge between $i$ and $j$ with $\infty$ whenever $a(i, j) a(j, i) \geq 4$. In this case it is, of course, not possible to reconstruct the values of $a(i, j)$ and $a(j, i)$ from the diagram $\Pi$.

Therefore, by convention, in this article for each edge between $i$ and $j$ with label $\infty$ we consider the values of $a(i, j)$ and $a(j, i)$ as part of the augmented Dynkin diagram: write $-a(i, j)$ between the vertex $i$ and the $\infty$ label and $-a(j, i)$ between the vertex $j$ and the $\infty$ label. In addition, an edge with $\infty$ label such that $a(i, j)$ and $a(j, i)$ have different parity gets directed $i \leftarrow j$, if $a(i, j)$ is even, and $i \rightarrow j$, if $a(i, j)$ is odd. See Figure 1 for an example.

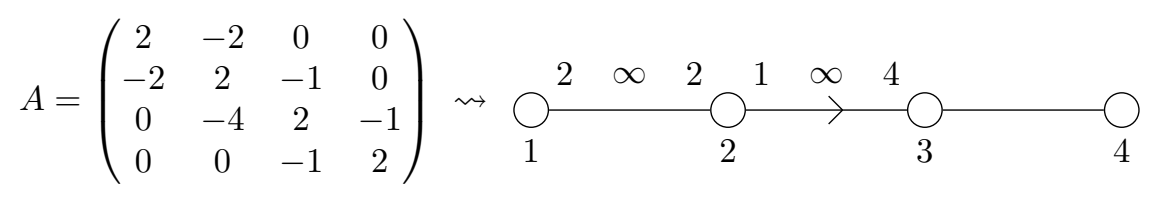

FiguRE 1. An augmented Dynkin diagram.

\section{The Groups $\mathrm{SO}(n)$ And $\mathrm{O}(n)$}

In this section we fix notation concerning the compact real orthogonal groups.

Definition 5.1. Given a quadratic space $(\mathbb{K}, V, q)$ with $\operatorname{dim}_{\mathbb{K}} V<\infty$, we set

$$
\mathrm{O}(q):=\{a \in \mathrm{GL}(V) \mid \forall v \in V: q(a v)=q(v)\}, \quad \mathrm{SO}(q):=\mathrm{O}(q) \cap \mathrm{SL}(V) .
$$


Given $n \in \mathbb{N}$, let $q_{n}: \mathbb{R}^{n} \rightarrow \mathbb{R}: x \mapsto \sum_{i=1}^{n} x_{i}^{2}$ be the standard quadratic form on $\mathbb{R}^{n}$, and

$$
\begin{aligned}
\mathrm{O}(n) & :=\left\{a \in \mathrm{GL}_{n}(\mathbb{R}) \mid a a^{t}=E_{n}\right\} \cong \mathrm{O}\left(q_{n}\right)=\mathrm{O}\left(-q_{n}\right), \\
\mathrm{SO}(n) & :=\mathrm{O}(n) \cap \mathrm{SL}_{n}(\mathbb{R}) \cong \mathrm{SO}\left(q_{n}\right)=\mathrm{SO}\left(-q_{n}\right) \unlhd \mathrm{O}\left(q_{n}\right)=\mathrm{O}\left(-q_{n}\right) .
\end{aligned}
$$

Since an element of $\mathrm{O}(n)$ has determinant 1 or -1 , we have $[\mathrm{O}(n): \mathrm{SO}(n)]=2$.

Notation 5.2. Let $n \in \mathbb{N}$ and let $\mathcal{E}=\left(e_{1}, \ldots, e_{n}\right)$ be the standard basis of $\mathbb{R}^{n}$. Given a subset $I \subseteq\{1, \ldots, n\}$, we set

$$
\mathcal{E}_{I}:=\left\{e_{i} \mid i \in I\right\}, \quad V_{I}:=\left\langle\mathcal{E}_{I}\right\rangle_{\mathbb{R}} \leq \mathbb{R}^{n}, \quad q_{I}:=q_{n \mid V_{I}}: V_{I} \rightarrow \mathbb{R} .
$$

There are canonical isomorphisms

$$
M_{\mathcal{E}}: \operatorname{End}\left(\mathbb{R}^{n}\right) \rightarrow \mathrm{M}_{n}(\mathbb{R}): a \mapsto M_{\mathcal{E}}(a) \quad \text { and } \quad M_{\mathcal{E}_{I}}: \operatorname{End}\left(V_{I}\right) \rightarrow M_{|I|}(\mathbb{R}): a \mapsto M_{\mathcal{E}_{I}}(a)
$$

that map an endomorphism into its transformation matrix with respect to the standard basis $\mathcal{E}$, resp. the basis $\mathcal{E}_{I}$. Moreover, there is a canonical embedding

$$
\varepsilon_{I}: \mathrm{O}\left(q_{I}\right) \rightarrow \mathrm{O}\left(q_{n}\right)
$$

inducing a canonical embedding

$$
M_{\mathcal{E}} \circ \varepsilon_{I} \circ M_{\mathcal{E}_{I}}^{-1}: \mathrm{O}(|I|) \rightarrow \mathrm{O}(n)
$$

which, by slight abuse of notation, we also denote by $\varepsilon_{I}$. We will furthermore use the same symbol for the (co)restriction of $\varepsilon_{I}$ to $\mathrm{SO}(\cdot)$. The most important application of this map in this article is for $|I|=2$ with $I=\{i, j\}$ providing the map

$$
\varepsilon_{i j}: \mathrm{SO}(2) \rightarrow \mathrm{SO}(n)
$$

6. The GRoups $\operatorname{Spin}(n)$ AND $\operatorname{Pin}(n)$

In this section we recall the compact real spin and pin groups. For a thorough treatment we refer to [LM89, Gal14, Mei13.

Definition 6.1. Let $(\mathbb{R}, V, q)$ be a quadratic space and let $T(V)=\bigoplus_{n>0} V^{\otimes n}$ be the tensor algebra of $V$. The identity $V^{\otimes 0}=\mathbb{R}$ provides a ring monomorphism $\mathbb{R} \rightarrow T(V)$, the identity $V^{\otimes 1}=V$ a vector space monomorphism $V \rightarrow T(V)$ that allow one to identify $\mathbb{R}, V$ with their respective images in $T(V)$. For

$$
\Im(q):=\langle v \otimes v-q(v) \mid v \in V\rangle
$$

define the Clifford algebra of $\boldsymbol{q}$ as

$$
\mathrm{Cl}(q):=T(V) / \mathfrak{I}(q)
$$

Moreover, let

$$
\mathrm{Cl}(q)^{*}:=\{x \in \mathrm{Cl}(q) \mid \exists y \in \mathrm{Cl}(q): x y=1\} .
$$

The transposition map is the involution

$$
\tau: \mathrm{Cl}(q) \rightarrow \mathrm{Cl}(q) \quad \text { induced by } \quad v_{1} \cdots v_{k} \mapsto v_{k} \cdots v_{1}, \quad v_{i} \in V,
$$

cf. [Mei13, Section 2.2.6], Gal14, Proposition 1.1]. The parity automorphism is the map

$$
\Pi: \mathrm{Cl}(q) \rightarrow \mathrm{Cl}(q) \quad \text { given by } \quad v_{1} \cdots v_{k} \mapsto(-1)^{k} \cdot v_{1} \cdots v_{k}, \quad v_{i} \in V,
$$

cf. [Mei13, Section 2.2.2, Section 3.1.1], Gal14, Proposition 1.2]. We set

$$
\mathrm{Cl}^{0}(q):=\{x \in \mathrm{Cl}(q) \mid \Pi(x)=x\} \quad \text { and } \quad \mathrm{Cl}^{1}(q):=\{x \in \mathrm{Cl}(q) \mid \Pi(x)=-x\},
$$

which yields a $\mathbb{Z}_{2}$-grading of $\mathrm{Cl}(q)$, i.e.,

$$
\mathrm{Cl}(q)=\mathrm{Cl}^{0}(q) \oplus \mathrm{Cl}^{1}(q) \quad \text { and } \quad \mathrm{Cl}^{i}(q) \mathrm{Cl}^{j}(q) \subseteq \mathrm{Cl}^{i+j}(q) \quad \text { for } i, j \in \mathbb{Z}_{2} .
$$

Furthermore, following [Gal14, Section 3.1], we define the Clifford conjugation

$$
\sigma: \mathrm{Cl}(q) \rightarrow \mathrm{Cl}(q): x \mapsto \bar{x}:=\tau \Pi(x)=\Pi \tau(x),
$$

and the spinor norm

$$
N: \mathrm{Cl}(q) \rightarrow \mathrm{Cl}(q): x \mapsto x \bar{x}
$$

Notation 6.2. In the following, $(\mathbb{R}, V, q)$ is an anisotropic quadratic space such that $\operatorname{dim}_{\mathbb{R}} V<\infty$. 
Definition 6.3. Given $x \in \mathrm{Cl}(q)^{*}$, the map

$$
\rho_{x}: \mathrm{Cl}(q) \rightarrow \mathrm{Cl}(q): y \mapsto \Pi(x) y x^{-1}
$$

is the twisted conjugation with respect to $x$. Using the canonical identification of $V$ with its image in $\mathrm{Cl}(q)$, we define

$$
\Gamma(q):=\left\{x \in \mathrm{Cl}(q)^{*} \mid \forall v \in V: \rho_{x}(v) \in V\right\}
$$

to be the Clifford group with respect to $\boldsymbol{q}$, cf. [Mei13, Section 3.1.1], Gal14, Definition 1.4]. We obtain a representation

$$
\rho: \Gamma(q) \rightarrow \mathrm{GL}(V): x \mapsto \rho_{x},
$$

which is the twisted adjoint representation.

Definition 6.4. Given $n \in \mathbb{N}$ and $V=\mathbb{R}^{n}$, we set

$$
\mathrm{Cl}(n):=\mathrm{Cl}\left(-q_{n}\right) \quad \text { and } \quad \Gamma(n):=\Gamma\left(-q_{n}\right) .
$$

Recall that $q_{n}$ is defined to be the standard quadratic form on $\mathbb{R}^{n}$, cf. Definition 5.1 .

Note that the literature one can also find the opposite sign convention.

Remark 6.5. (a) Let $n \in \mathbb{N}$ and let $e_{1}, \ldots, e_{n}$ be the standard basis of $\mathbb{R}^{n}$. Then the following hold in $\mathrm{Cl}(n)$ for $1 \leq i \neq j \leq n$ :

$$
\begin{aligned}
e_{i}^{2} & =-1, \\
e_{i} e_{j} & =-e_{j} e_{i}, \\
\left(e_{i} e_{j}\right)^{2} & =-1 .
\end{aligned}
$$

The first identity is immediate from the definition. The second identity follows from polarization, as in the tensor algebra $T\left(\mathbb{R}^{n}\right)$ one has

$$
\begin{aligned}
\Im\left(q_{n}\right) & \ni\left(e_{i}+e_{j}\right) \otimes\left(e_{i}+e_{j}\right)-q\left(e_{i}+e_{j}\right) \\
& =e_{i} \otimes e_{i}+e_{i} \otimes e_{j}+e_{j} \otimes e_{i}+e_{j} \otimes e_{j}-q\left(e_{i}\right)-q\left(e_{j}\right)-2 b\left(e_{i}, e_{j}\right) \\
& =e_{i} \otimes e_{j}+e_{j} \otimes e_{i},
\end{aligned}
$$

where $b(\cdot, \cdot)$ denotes the bilinear form associated to $q_{n}$. The third identity is immediate from the first two.

(b) One has $\mathrm{Cl}^{0}(3) \cong \mathbb{H}$, where $\mathbb{H}$ denotes the quaternions. Indeed, given a basis $e_{1}, e_{2}, e_{3}$ of $\mathbb{R}^{3}$, a basis of $\mathrm{Cl}^{0}(3)$, considered as an $\mathbb{R}$-vector space, is given by $1, e_{1} e_{2}, e_{2} e_{3}, e_{3} e_{1}$. By (a) the latter three basis elements square to -1 and anticommute with one another. Note, furthermore, that under this isomorphism the Clifford conjugation is transformed into the standard involution of the quaternions and, consequently, the the spinor norm into the norm of the quaternions.

Lemma 6.6. The map $N: \mathrm{Cl}(q) \rightarrow \mathrm{Cl}(q)$ induces a homomorphism

$$
N: \Gamma(q) \rightarrow \mathbb{R}^{*}
$$

such that

$$
\forall x \in \Gamma(q): \quad N(\Pi(x))=N(x) .
$$

Proof. Cf. Gal14, Proposition 1.9].

Definition 6.7. The group

$$
\operatorname{Pin}(q):=\{x \in \Gamma(q) \mid N(x)=1\} \leq \Gamma(q)
$$

is the pin group with respect to $q$, and

$$
\operatorname{Spin}(q):=\operatorname{Pin}(q) \cap \mathrm{Cl}^{0}(q) \leq \operatorname{Pin}(q)
$$

is the spin group with respect to $\boldsymbol{q}$. By Lemma 6.6 and the $\mathbb{Z}_{2}$-grading of $\mathrm{Cl}(q)$, the sets $\operatorname{Pin}(q)$ and $\operatorname{Spin}(q)$ are indeed subgroups of $\Gamma(q)$. Given $n \in \mathbb{N}$, define

$$
\operatorname{Pin}(n):=\operatorname{Pin}\left(-q_{n}\right) \quad \text { and } \quad \operatorname{Spin}(n):=\operatorname{Spin}\left(-q_{n}\right) .
$$

Theorem 6.8. The following hold:

(a) One has $[\operatorname{Pin}(q): \operatorname{Spin}(q)]=2$ and $\operatorname{Spin}(q)=\rho^{-1}(\operatorname{SO}(q))$. 
(b) The twisted adjoint representation $\rho: \Gamma(q) \rightarrow \mathrm{GL}(V)$ induces an epimorphism $\rho: \operatorname{Pin}(q) \rightarrow \mathrm{O}(q)$. In particular, given $n \in \mathbb{N}$, we obtain epimorphisms

$$
\rho_{n}:=M_{\mathcal{E}} \circ \rho: \operatorname{Pin}(n) \rightarrow \mathrm{O}(n), \quad \quad \rho_{n}:=M_{\mathcal{E}} \circ \rho: \operatorname{Spin}(n) \rightarrow \operatorname{SO}(n)
$$

with $\operatorname{ker}\left(\rho_{n}\right)=\{ \pm 1\}$ in both cases.

(c) The group $\operatorname{Spin}(q)$ is a double cover of the group $\mathrm{SO}(q)$.

Proof. See Gal14, Theorem 1.11].

\section{Remark 6.9.}

(a) By slight abuse of notation, suppressing the choice of basis, we will also sometimes denote the map $\rho_{n}$ by $\rho$.

(b) Let $H_{1} \leq \operatorname{Spin}(n)$ and $H_{2} \leq \operatorname{Pin}(n)$ be such that $-1 \in H_{1}$ and $-1 \in H_{2}$, respectively, and let $\tilde{H}_{i}:=\rho_{n}\left(H_{i}\right)$. Then we have $H_{i}=\rho_{n}^{-1}\left(\tilde{H}_{i}\right)$. We will explicitly determine these groups for some canonical subgroups of $\mathrm{SO}(n)$ and $\mathrm{O}(n)$.

Lemma 6.10. Let $n \in \mathbb{N}$ and $I \subseteq\{1, \ldots, n\}$. Then there is a canonical embedding $\tilde{\varepsilon}_{I}: \operatorname{Pin}\left(-q_{I}\right) \rightarrow$ $\operatorname{Pin}\left(-q_{n}\right)$ satisfying

$$
\forall x \in \mathrm{Cl}\left(-q_{I}\right):\left.\quad \rho_{\tilde{\varepsilon}_{I}(x)}\right|_{\mid V_{I}}=\varepsilon_{I} \circ \rho_{x}
$$

such that the following diagram commutes:

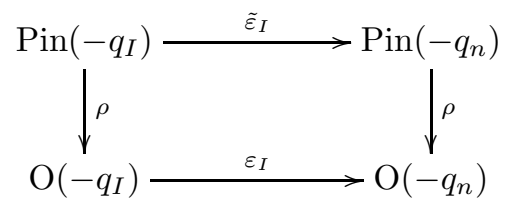

In analogy to Notation 5.2 we will use the same symbol for the (co)restriction of $\tilde{\varepsilon}_{I}$ to $\operatorname{Spin}(\cdot)$. The most important application of this map in this article is for $|I|=2$ with $I=\{i, j\}$ providing the map

$$
\tilde{\varepsilon}_{i j}: \operatorname{Spin}(2) \rightarrow \operatorname{Spin}(n) .
$$

Proof. Let $x \in \Gamma\left(-q_{I}\right)$. By definition,

$$
\forall v \in V_{I}: \quad \rho_{\tilde{\varepsilon}_{I}(x)}(v) \in V_{I} \subseteq \mathbb{R}^{n} .
$$

Since $e_{i} e_{j}=-e_{j} e_{i}$ for all $i \neq j \in I$ by Remark 6.5)(a), for each $\mathbb{R}$-basis vector $y=e_{j_{1}} \cdots e_{j_{k}}$ of $\tilde{\varepsilon}_{I}\left(\mathrm{Cl}\left(-q_{I}\right)\right)$ and all $i \in\{1, \ldots, n\} \backslash I$ one has

$$
\Pi(y) e_{i}=e_{i} y
$$

Hence

and, thus, for all $i \in\{1, \ldots, n\} \backslash I$

$$
\Pi\left(\tilde{\varepsilon}_{I}(x)\right) e_{i}=e_{i} \tilde{\varepsilon}_{I}(x)
$$

$$
\rho_{\tilde{\varepsilon}_{I}(x)}\left(e_{i}\right)=\Pi\left(\tilde{\varepsilon}_{I}(x)\right) e_{i} \tilde{\varepsilon}_{I}(x)^{-1}=e_{i} \in \mathbb{R}^{n} .
$$

As $\tilde{\varepsilon}_{I}\left(\mathrm{Cl}\left(-q_{I}\right)\right)$ is generated as an $\mathbb{R}$-algebra by the set $\left\{e_{i} \mid i \in I\right\}$, we in particular have

$$
\rho \circ \tilde{\varepsilon}_{I}=\varepsilon_{I} \circ \rho .
$$

Therefore $\varepsilon_{I}(x) \in \Gamma\left(-q_{n}\right)$. Finally,

$$
N\left(\tilde{\varepsilon}_{I}(x)\right)=\tilde{\varepsilon}_{I}(N(x))=\tilde{\varepsilon}_{I}(1)=1,
$$

whence

$$
\tilde{\varepsilon}_{I}(x) \in \operatorname{Pin}\left(-q_{n}\right) .
$$

Remark 6.11. Since $\tilde{\varepsilon}_{I}\left(\operatorname{Spin}\left(-q_{I}\right)\right)=\left\langle e_{i} e_{j} \mid i \neq j \in I\right\rangle \subseteq \mathrm{Cl}^{0}\left(-q_{n}\right)$, one has

$$
\tilde{\varepsilon}_{I}\left(\operatorname{Spin}\left(-q_{I}\right)\right) \subseteq \operatorname{Pin}\left(-q_{n}\right) \cap \mathrm{Cl}^{0}\left(-q_{n}\right)=\operatorname{Spin}\left(-q_{n}\right) .
$$

Consequence 6.12. Let $n \in \mathbb{N}$ and $I \subseteq\{1, \ldots, n\}$. Then

$$
\rho_{n}^{-1}\left(\varepsilon_{I}(\mathrm{O}(|I|))\right)=\tilde{\varepsilon}_{I}\left(\operatorname{Pin}\left(-q_{I}\right)\right) \quad \text { and } \quad \rho_{n}^{-1}\left(\varepsilon_{I}(\operatorname{SO}(|I|))\right)=\tilde{\varepsilon}_{I}\left(\operatorname{Spin}\left(-q_{I}\right)\right) .
$$

Proof. By Lemma 6.10, one has $\rho_{n} \tilde{\varepsilon}_{I}\left(\operatorname{Pin}\left(-q_{I}\right)\right)=\varepsilon_{I}(\mathrm{O}(|I|))$ and $\rho_{n} \tilde{\varepsilon}_{I}\left(\operatorname{Spin}\left(-q_{I}\right)\right)=\varepsilon_{I}(\operatorname{SO}(|I|))$, thus the assertion results from Remark $6.9(\mathrm{~b})$. 
Remark 6.13. Let $n \in \mathbb{N}$, let $I \subseteq\{1, \ldots, n\}$ and let $m:=|I|$. Then there exists an isomorphism $i: \operatorname{Pin}(m) \rightarrow \operatorname{Pin}\left(-q_{I}\right)$ such that the following diagram commutes:

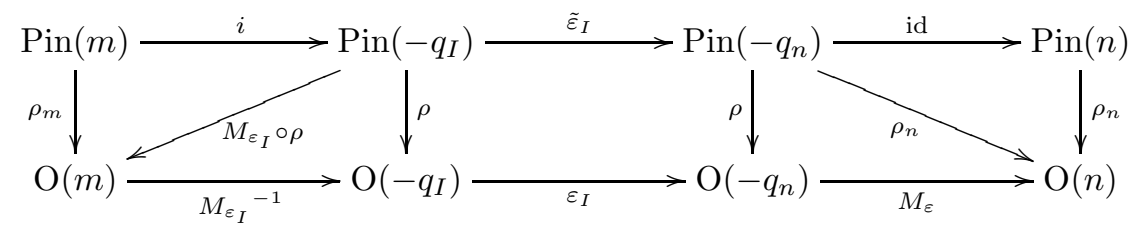

As in 5.2 we slightly abuse notation and also write $\tilde{\varepsilon}_{I}$ for the map id $\circ \tilde{\varepsilon}_{I} \circ i: \operatorname{Pin}(m) \rightarrow \operatorname{Pin}(n)$ and $\varepsilon_{I}$ for the map $M_{\varepsilon} \circ \varepsilon_{I} \circ M_{\varepsilon_{I}}{ }^{-1}: \mathrm{O}(m) \rightarrow \mathrm{O}(n)$. Consequently, we obtain the following commutative diagram:

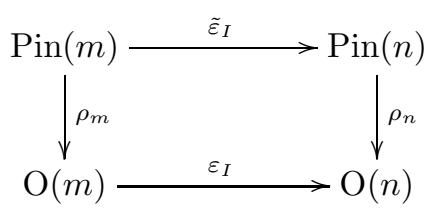

Remark 6.14. According to Gal14, Corollary 1.12], the group $\operatorname{Pin}(n)$ is generated by the set

$$
\left\{v \in \mathbb{R}^{n} \mid N(v)=1\right\}
$$

and each element of the group $\operatorname{Spin}(n)$ can be written as a product of an even number of elements from this set. That is, each element $g \in \operatorname{Spin}(2)$ is of the form

$$
g=\prod_{i=1}^{2 k}\left(a_{i} e_{1}+b_{i} e_{2}\right)=\prod_{i=1}^{k}\left(\left(a_{2 i-1} a_{2 i}+b_{2 i-1} b_{2 i}\right)+\left(a_{2 i-1} b_{2 i}-a_{2 i} b_{2 i-1}\right) e_{1} e_{2}\right)=: a+b e_{1} e_{2} .
$$

The requirement $a_{i} e_{1}+b_{i} e_{2} \in\left\{v \in \mathbb{R}^{n} \mid N(v)=1\right\}$ is equivalent to

$$
a_{i}^{2}+b_{i}^{2}=\left(a_{i} e_{1}+b_{i} e_{2}\right)\left(-a_{i} e_{1}-b_{i} e_{2}\right)=\left(a_{i} e_{1}+b_{i} e_{2}\right) \overline{\left(a_{i} e_{1}+b_{i} e_{2}\right)}=N\left(a_{i} e_{1}+b_{i} e_{2}\right)=1 .
$$

Morever,

$$
1=N(g)=N\left(a+b e_{1} e_{2}\right)=\left(a+b e_{1} e_{2}\right) \overline{\left(a+b e_{1} e_{2}\right)}=\left(a+b e_{1} e_{2}\right)\left(a+b e_{2} e_{1}\right)=a^{2}+b^{2} .
$$

Certainly, $\operatorname{Spin}(2)$ contains all elements of the form $a+b e_{1} e_{2}$ with $a^{2}+b^{2}=1$, i.e., one obtains

$$
\operatorname{Spin}(2)=\left\{\cos (\alpha)+\sin (\alpha) e_{1} e_{2} \mid \alpha \in \mathbb{R}\right\}
$$

One has

$$
\left(\cos (\alpha)+\sin (\alpha) e_{1} e_{2}\right)^{-1}=\cos (\alpha)-\sin (\alpha) e_{1} e_{2}=\cos (-\alpha)+\sin (-\alpha) e_{1} e_{2},
$$

i.e., the map

$$
\mathbb{R} \rightarrow \operatorname{Spin}(2): \alpha \mapsto \cos (\alpha)+\sin (\alpha) e_{1} e_{2}
$$

is a group homomorphism from the real numbers onto the circle group. The twisted adjoint representation $\rho_{2}$ maps the element $\cos (\alpha)+\sin (\alpha) e_{1} e_{2} \in \operatorname{Spin}(2)$ to the transformation

$$
\begin{aligned}
x_{1} e_{1}+x_{2} e_{2} \mapsto & \left(\cos (\alpha)+\sin (\alpha) e_{1} e_{2}\right)\left(x_{1} e_{1}+x_{2} e_{2}\right)\left(\cos (\alpha)-\sin (\alpha) e_{1} e_{2}\right) \\
= & x_{1}\left(\cos (\alpha)^{2}-\sin (\alpha)^{2}\right) e_{1}-2 x_{2} \cos (\alpha) \sin (\alpha) e_{1} \\
& +2 x_{1} \cos (\alpha) \sin (\alpha) e_{2}+x_{2}\left(\cos (\alpha)^{2}-\sin (\alpha)^{2}\right) e_{2} \\
= & \left(x_{1} \cos (2 \alpha)-x_{2} \sin (2 \alpha)\right) e_{1}+\left(x_{1} \sin (2 \alpha)+x_{2} \cos (2 \alpha)\right) e_{2},
\end{aligned}
$$

i.e., the rotation of the euclidean plane $\mathbb{R}^{2}$ by the angle $2 \alpha$. In other words, $\rho_{2}$ is the double cover of the circle group by itself, cf. Theorem 6.8(b).

Similarly, each element $g \in \operatorname{Spin}(3)$ is of the form

$$
g=\prod_{i=1}^{2 k}\left(a_{i} e_{1}+b_{i} e_{2}+c_{i} e_{3}\right)=a+b e_{1} e_{2}+c e_{2} e_{3}+d e_{3} e_{1}
$$

and each element $h \in \operatorname{Spin}(4)$ of the form

$h=\prod_{i=1}^{2 k}\left(a_{i} e_{1}+b_{i} e_{2}+c_{i} e_{3}+d_{i} e_{4}\right)=h_{1}+h_{2} e_{1} e_{2}+h_{3} e_{2} e_{3}+h_{4} e_{3} e_{1}+h_{5} e_{1} e_{2} e_{3} e_{4}+h_{6} e_{4} e_{3}+h_{7} e_{4} e_{1}+h_{8} e_{4} e_{2}$ 


\section{The ISOMORPHISM $\operatorname{Spin}(4) \cong \operatorname{Spin}(3) \times \operatorname{Spin}(3)$}

In this section we recall special isomorphisms admitted by the groups $\operatorname{Spin}(3)$ and $\operatorname{Spin}(4)$. This structural information will only become relevant in Part III (Sections 12 and 13) of this article.

Definition 7.1. Denote by

$$
\mathbb{H}:=\{a+b i+c j+d k \mid a, b, c, d \in \mathbb{R}\}
$$

the real quaternions, identify $\mathbb{R}$ with the centre of $\mathbb{H}$ via $\mathbb{R} \rightarrow \mathbb{H}: a \mapsto a$, let

$$
\ulcorner: \mathbb{H} \rightarrow \mathbb{H}: x=a+b i+c j+d k \mapsto \bar{x}=a-b i-c j-d k
$$

be the standard involution, and let

$$
\mathrm{U}_{1}(\mathbb{H}):=\left\{x \in \mathbb{H} \mid x \bar{x}=1_{\mathbb{H}}\right\}
$$

be the group of unit quaternions.

Remark 7.2. By [Gal14, Section 1.4] one has

$$
\operatorname{Spin}(3) \cong \mathrm{U}_{1}(\mathbb{H}) \quad \text { and } \quad \operatorname{Spin}(4) \cong \operatorname{Spin}(3) \times \operatorname{Spin}(3) \cong \mathrm{U}_{1}(\mathbb{H}) \times \mathrm{U}_{1}(\mathbb{H}) .
$$

The isomorphism $\operatorname{Spin}(3) \cong \mathrm{U}_{1}(\mathbb{H})$ in fact is an immediate consequence of the isomorphism $\mathrm{Cl}^{0}(3) \cong \mathbb{H}$ from Remark 6.5 (b) plus the observation that this isomorphism transforms the spinor norm into the norm of the quaternions.

A canonical isomorphism $\operatorname{Spin}(4) \cong \operatorname{Spin}(3) \times \operatorname{Spin}(3) \cong \mathrm{U}_{1}(\mathbb{H}) \times \mathrm{U}_{1}(\mathbb{H})$ can be described as follows (see [Gal14, Section 1.4]). By Remark 6.14 each element of Spin(4) is of the form

$$
a+b e_{1} e_{2}+c e_{2} e_{3}+d e_{3} e_{1}+a^{\prime} e_{1} e_{2} e_{3} e_{4}+b^{\prime} e_{4} e_{3}+c^{\prime} e_{4} e_{1}+d^{\prime} e_{4} e_{2} .
$$

For

$$
i:=e_{1} e_{2}, \quad j:=e_{2} e_{3}, \quad k:=e_{3} e_{1}, \quad \mathbb{I}:=e_{1} e_{2} e_{3} e_{4}, \quad i^{\prime}:=e_{4} e_{3}, \quad j^{\prime}:=e_{4} e_{1}, \quad k^{\prime}:=e_{4} e_{2}
$$

one has

$$
\begin{aligned}
i j & =k, & j k & =i, \\
i \mathbb{I} & =\mathbb{I} i=i^{\prime}, & k i & =j, \\
i^{2} & =j^{2}=k^{2}=-1, & k \mathbb{I} & =\mathbb{I} k=k^{\prime}, \\
\mathbb{I}^{2} & =1, & \sigma(\mathbb{I}) & =\mathbb{I},
\end{aligned}
$$

where $\sigma(\mathbb{I})$ denotes the Clifford conjugate of $\mathbb{I}$, cf. Definition 6.1 We conclude that for every $x \in \operatorname{Spin}(4)$ there exist uniquely determined $u=a+b i+c j+d k, v=a^{\prime}+b^{\prime} i+c^{\prime} j+d^{\prime} k \in \mathbb{H}$ such that

$$
x=u+\mathbb{I} v .
$$

One computes

$$
N(x)=N(u+\mathbb{I} v)=(u+\mathbb{I} v)(\bar{u}+\mathbb{I} \bar{v})=u \bar{u}+v \bar{v}+\mathbb{I}(u \bar{v}+v \bar{u}),
$$

i.e.,

$$
N(x)=1 \Longleftrightarrow u \bar{u}+v \bar{v}=1 \text { and } u \bar{v}+v \bar{u}=0 .
$$

Hence, for $1=N(x)=N(u+\mathbb{I} v)$, one has

$$
\begin{aligned}
& N(u+v)=(u+v)(\bar{u}+\bar{v})=1, \\
& N(u-v)=(u-v)(\bar{u}-\bar{v})=1 .
\end{aligned}
$$

That is, the map

$$
\operatorname{Spin}(4) \rightarrow \operatorname{Spin}(3) \times \operatorname{Spin}(3): u+\mathbb{I} v \mapsto(u+v, u-v)
$$

is a well-defined bijection and, since

$$
\begin{aligned}
(u+\mathbb{I} v)\left(u^{\prime}+\mathbb{I} v^{\prime}\right) & =u u^{\prime}+v v^{\prime}+\mathbb{I}\left(u v^{\prime}+v u^{\prime}\right), \\
(u+v, u-v)\left(u^{\prime}+v^{\prime}, u^{\prime}-v^{\prime}\right) & =\left(u u^{\prime}+v v^{\prime}+u v^{\prime}+v u^{\prime}, u u^{\prime}+v v^{\prime}-\left(u v^{\prime}+v u^{\prime}\right)\right),
\end{aligned}
$$

in fact an isomorphism of groups.

Consequently, there exist a group epimorphism

$$
\tilde{\eta}: \operatorname{Spin}(4) \rightarrow \operatorname{Spin}(3): u+\mathbb{I} v \mapsto u+v .
$$


Remark 7.3. Using this isomorphism $\operatorname{Spin}(4) \cong \operatorname{Spin}(3) \times \operatorname{Spin}(3) \cong U_{1}(\mathbb{H}) \times U_{1}(\mathbb{H})$ there exists a natural homomorphism

$$
\operatorname{Spin}(4) \rightarrow \mathrm{SO}(\mathbb{H}) \cong \mathrm{SO}(4):(a, b) \mapsto\left(x \mapsto a x b^{-1}\right)
$$

Note that the restrictions $(a, 1) \mapsto(x \mapsto a x)$ and $(1, b) \mapsto\left(x \mapsto x b^{-1}\right)$ both are injections of $\operatorname{Spin}(3) \cong$ $\mathrm{U}_{1}(\mathbb{H})$ into $\mathrm{GL}(\mathbb{H}) \cong(\mathbb{H} \backslash\{0\}, \cdot)$, in fact into $\mathrm{SO}(\mathbb{H})$, as the norm is multiplicative. Since the kernel of this action has order two, the homomorphism $\mathrm{Spin}(4) \rightarrow \mathrm{SO}(\mathbb{H}) \cong \mathrm{SO}(4)$ must be onto by Proposition 6.8 . We conclude that the group $\mathrm{SO}(4)$ is isomorphic to the group consisting of the maps

$$
\mathbb{H} \rightarrow \mathbb{H}: x \mapsto a x b^{-1} \quad \text { for } a, b \in \mathrm{U}_{1}(\mathbb{H}) ;
$$

for an alternative proof see $\left[\mathrm{SBG}^{+95}\right.$, Lemma 11.22].

A similar argument (or a direct computation using the twisted adjoint representation) shows that the natural homomorphism

$$
\operatorname{Spin}(3) \rightarrow \mathrm{SO}\left(\langle i, j, k\rangle_{\mathbb{R}}\right) \cong \mathrm{SO}(3): a \mapsto\left(x \mapsto a x a^{-1}\right)
$$

is an epimorphism and, thus, that the group $\mathrm{SO}(3)$ is isomorphic to the group consisting of the maps

$$
\mathbb{H} \rightarrow \mathbb{H}: x \mapsto a x a^{-1} \quad \text { for } a \in \mathrm{U}_{1}(\mathbb{H}) ;
$$

see also $\left[\mathrm{SBG}^{+} 95\right.$, Lemma 11.24].

Remark 7.4. There also exists a group epimorphism

$$
\eta: \mathrm{SO}(4) \rightarrow \mathrm{SO}(3)
$$

induced by the map

$$
\begin{aligned}
\mathrm{SO}(4) \cong\left\{\mathbb{H} \rightarrow \mathbb{H}: x \mapsto a x b^{-1} \mid a, b \in \mathrm{U}_{1}(\mathbb{H})\right\} & \rightarrow\left\{\mathbb{H} \rightarrow \mathbb{H}\left|x \mapsto a x a^{-1}\right| a \in \mathrm{U}_{1}(\mathbb{H})\right\} \cong \mathrm{SO}(3) \\
\left(x \mapsto a x b^{-1}\right) & \mapsto\left(x \mapsto a x a^{-1}\right)
\end{aligned}
$$

Altogether, one obtains the following commutative diagram:

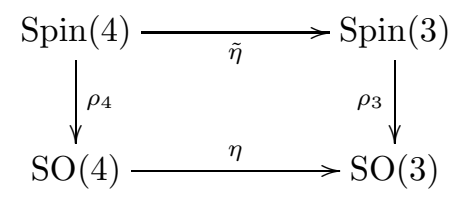

\section{Lifting AUtomorphism from $\mathrm{SO}(n)$ TO $\operatorname{Spin}(n)$}

Notation 8.1. For $\mathrm{SO}(2) \times \mathrm{SO}(2)=\{(a, b) \mid a, b \in \mathrm{SO}(2)\}$ let

$$
\iota_{1}: \mathrm{SO}(2) \rightarrow \mathrm{SO}(2) \times \mathrm{SO}(2): x \mapsto(x, 1), \quad \iota_{2}: \mathrm{SO}(2) \rightarrow \mathrm{SO}(2) \times \mathrm{SO}(2): x \mapsto(1, x) .
$$

Similarly, for $\operatorname{Spin}(2) \times \operatorname{Spin}(2)=\{(a, b) \mid a, b \in \operatorname{Spin}(2)\}$ let

$$
\tilde{\iota}_{1}: \operatorname{Spin}(2) \rightarrow \operatorname{Spin}(2) \times \operatorname{Spin}(2): x \mapsto(x, 1), \quad \tilde{\iota}_{2}: \operatorname{Spin}(2) \rightarrow \operatorname{Spin}(2) \times \operatorname{Spin}(2): x \mapsto(1, x) .
$$

Moreover, define

$$
\rho_{2} \times \rho_{2}: \operatorname{Spin}(2) \times \operatorname{Spin}(2) \rightarrow \mathrm{SO}(2) \times \mathrm{SO}(2):(a, b) \mapsto\left(\rho_{2}(a), \rho_{2}(b)\right) .
$$

Hence

Furthermore, let

$$
\left(\rho_{2} \times \rho_{2}\right) \circ \tilde{\iota}_{1}=\iota_{1} \circ \rho_{2}, \quad\left(\rho_{2} \times \rho_{2}\right) \circ \tilde{\iota}_{2}=\iota_{2} \circ \rho_{2}
$$

$$
\pi: \operatorname{Spin}(2) \times \operatorname{Spin}(2) \rightarrow \operatorname{Spin}(2) \times \operatorname{Spin}(2) /\langle(-1,-1)\rangle
$$

be the canonical projection. By the homomorphism theorem of groups the map $\rho_{2} \times \rho_{2}$ factors through $\operatorname{Spin}(2) \times \operatorname{Spin}(2) /\langle(-1,-1)\rangle$ and induces the following commutative diagram:

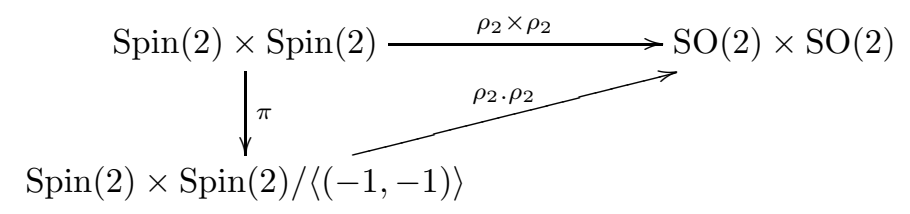

For $\alpha \in \mathbb{R}$ let

$$
D(\alpha):=\left(\begin{array}{cc}
\cos (\alpha) & \sin (\alpha) \\
-\sin (\alpha) & \cos (\alpha)
\end{array}\right) \in \operatorname{SO}(2) \quad \text { and } \quad S(\alpha):=\cos (\alpha)+\sin (\alpha) e_{1} e_{2} \in \operatorname{Spin}(2)
$$


Then $\operatorname{Spin}(2)=\{S(\alpha) \mid \alpha \in \mathbb{R}\}$ and $\mathrm{SO}(2)=\{D(\alpha) \mid \alpha \in \mathbb{R}\}$ and there is a continuous group isomorphism

$$
\psi: \mathrm{SO}(2) \rightarrow \operatorname{Spin}(2): D(\alpha) \mapsto S(\alpha) .
$$

By the computation in Remark 6.14 the epimorphism $\rho_{2}$ from Theorem 6.8 satisfies $\rho_{2}=\operatorname{sq} \circ \psi^{-1}$, i.e.

$$
\rho_{2}: \operatorname{Spin}(2) \rightarrow \mathrm{SO}(2): S(\alpha) \mapsto D(2 \alpha) .
$$

Proposition 8.2. Given an automorphism $\gamma \in \operatorname{Aut}(\mathrm{SO}(2))$, there is a unique automorphism $\tilde{\gamma} \in$ $\operatorname{Aut}(\operatorname{Spin}(2))$ such that $\rho_{2} \circ \tilde{\gamma}=\gamma \circ \rho_{2}$. Moreover, $\gamma$ is continuous if and only if $\tilde{\gamma}$ is continuous.

Proof. Define $\tilde{\gamma}:=\psi \circ \gamma \circ \psi^{-1}$. Then

$$
\rho_{2} \circ \tilde{\gamma}=\left(\operatorname{sq} \circ \psi^{-1}\right) \circ\left(\psi \circ \gamma \circ \psi^{-1}\right)=\operatorname{sq} \circ \gamma \circ \psi^{-1}=\gamma \circ \mathrm{sq} \circ \psi^{-1}=\gamma \circ \rho_{2}
$$

Uniqueness follows as $\operatorname{Aut}(\mathrm{SO}(2)) \rightarrow \operatorname{Aut}(\operatorname{Spin}(2)): \gamma \mapsto \psi \circ \gamma \circ \psi^{-1}$ is an isomorphism.

Corollary 8.3. Given an automorphism $\gamma \in \operatorname{Aut}(\mathrm{SO}(2) \times \mathrm{SO}(2))$, there is a unique automorphism $\tilde{\gamma} \in \operatorname{Aut}(\operatorname{Spin}(2) \times \operatorname{Spin}(2))$ such that

$$
\left(\rho_{2} \times \rho_{2}\right) \circ \tilde{\gamma}=\gamma \circ\left(\rho_{2} \times \rho_{2}\right) .
$$

Proof. Let $\tilde{\gamma}:=\psi \circ \gamma \circ \psi^{-1}$, where

$$
\psi: \mathrm{SO}(2) \times \mathrm{SO}(2) \rightarrow \operatorname{Spin}(2) \times \operatorname{Spin}(2),(D(\alpha), D(\beta)) \mapsto(S(\alpha), S(\beta)),
$$

and observe that $\rho_{2} \times \rho_{2}=\mathrm{sq} \circ \psi^{-1}$. Proceed as in the proof of Proposition 8.2 .

Proposition 8.4. Let $n \geq 3$. Given an automorphism $\gamma \in \operatorname{Aut}(\mathrm{SO}(n))$, there is a unique automorphism $\tilde{\gamma} \in \operatorname{Aut}(\operatorname{Spin}(n))$ such that

$$
\rho_{n} \circ \tilde{\gamma}=\gamma \circ \rho_{n}
$$

Proof. For $n \geq 3$, both $\mathrm{SO}(n)$ and $\operatorname{Spin}(n)$ are perfect, cf. [HM98, Corollary 6.56]. By Theorem 6.8(b) the group $\operatorname{Spin}(n)$ is a central extension of $\operatorname{SO}(n)$. Since $\operatorname{Spin}(n)$ is simply connected (see, e.g., Gal14, Section 1.8], it in fact is the universal central extension of $\operatorname{SO}(n)$.

The universal property of universal central extensions (cf. e.g. [HO89, Section 1.4C]) yields the claim: Indeed, there are unique homomorphisms $\tilde{\gamma}, \tilde{\gamma}^{\prime}: \operatorname{Spin}(n) \rightarrow \operatorname{Spin}(n)$ such that

$$
\gamma \circ \rho_{n}=\rho_{n} \circ \tilde{\gamma} \text { and } \gamma^{-1} \circ \rho_{n}=\rho_{n} \circ \tilde{\gamma}^{\prime} \text {. }
$$

Hence

$$
\rho_{n} \circ \tilde{\gamma} \circ \tilde{\gamma}^{\prime}=\gamma \circ \rho_{n} \circ \tilde{\gamma}^{\prime}=\gamma \circ \gamma^{-1} \circ \rho_{n}=\rho_{n}
$$

and, similarly,

$$
\rho_{n} \circ \tilde{\gamma}^{\prime} \circ \tilde{\gamma}=\rho_{n} .
$$

The universal property therefore implies $\tilde{\gamma} \circ \tilde{\gamma}^{\prime}=\mathrm{id}=\tilde{\gamma}^{\prime} \circ \tilde{\gamma}$, i.e., $\tilde{\gamma}$ is an automorphism.

In fact, all automorphisms are continuous by van der Waerden's Continuity Theorem, cf. [HM98, Theorem 5.64].

For the following proposition recall the definitions of $\varepsilon_{i j}$ in Notation 5.2 and of $\tilde{\varepsilon}_{i j}$ in Lemma 6.10,

Proposition 8.5. Let $\phi: \operatorname{Spin}(2) \rightarrow \operatorname{Spin}(n)$ be a homomorphism such that

$$
\operatorname{ker}\left(\rho_{n} \circ \phi\right)=\{1,-1\} \quad \text { and } \quad \rho_{n} \circ \phi=\varepsilon_{i j} \circ \rho_{2}
$$

for some $i \neq j \in I$. Then $\phi=\tilde{\varepsilon}_{i j}$.

Proof. By Consequence 6.12 one has

$$
\phi(\operatorname{Spin}(2)) \subseteq\left(\rho_{n}{ }^{-1} \circ \varepsilon_{i j} \circ \rho_{2}\right)(\operatorname{Spin}(2))=\rho_{n}{ }^{-1}\left(\varepsilon_{i j}(\operatorname{SO}(2))\right)=\tilde{\varepsilon}_{i j}(\operatorname{Spin}(2)) .
$$

By hypothesis $\operatorname{ker} \phi \subseteq\{1,-1\}$. If $-1 \in \operatorname{ker} \phi$, then $1=\phi(-1)=\phi(S(\pi))=\phi\left(S\left(\frac{\pi}{2}\right)\right)^{2}$, i.e., $\phi\left(S\left(\frac{\pi}{2}\right)\right) \in$ $\{1,-1\}$, whence $S\left(\frac{\pi}{2}\right) \in \operatorname{ker}\left(\rho_{n} \circ \phi\right)$, a contradiction. Consequently, $\phi$ is a monomorphism. 
Consider the following commuting diagram:

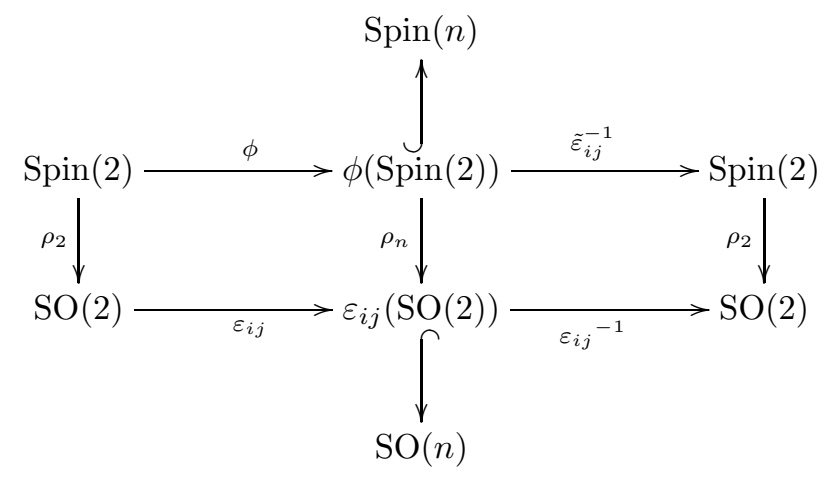

One has $\rho_{2} \circ \tilde{\varepsilon}_{i j}^{-1} \circ \phi=\rho_{2}=\mathrm{id} \circ \rho_{2}$. Since $\phi$ is injective, the map $\tilde{\varepsilon}_{i j}^{-1} \circ \phi$ is an automorphism of $\operatorname{Spin}(2)$. Hence Proposition 8.2 implies $\tilde{\varepsilon}_{i j}^{-1} \circ \phi=\mathrm{id}$.

\section{Part II. Simply laced diagrams}

\section{SO(2)-AMALGAMS OF SIMPLY LACED TYPE}

In this section we discuss amalgamation results for compact real orthogonal groups. The results and exposition are similar to Bor84, Gra06]. The key difference is that the amalgams in the present article are constructed starting with the circle group $\mathrm{SO}(2)$ instead of the perfect group $\mathrm{SU}(2)$. This leads to some subtle complications that we will need to address below.

Recall the maps $\varepsilon_{12}, \varepsilon_{23}: \mathrm{SO}(2) \rightarrow \mathrm{SO}(3)$ from Notation 5.2 and the maps $\iota_{1}, \iota_{2}: \mathrm{SO}(2) \rightarrow \mathrm{SO}(2) \times$ $\mathrm{SO}(2)$ from Notation 8.1

Definition 9.1. Let $\Pi$ be a simply laced diagram with labelling $\sigma: I \rightarrow V$. An $\mathbf{S O}(2)$-amalgam with respect to $\Pi$ and $\sigma$ is an amalgam $\mathcal{A}=\left\{G_{i j}, \phi_{i j}^{i} \mid i \neq j \in I\right\}$ such that

$$
\forall i \neq j \in I: \quad G_{i j}= \begin{cases}\mathrm{SO}(3), & \text { if }\{i, j\}^{\sigma} \in E(\Pi), \\ \mathrm{SO}(2) \times \mathrm{SO}(2), & \text { if }\{i, j\}^{\sigma} \notin E(\Pi),\end{cases}
$$

and for $i<j \in I$,

$$
\phi_{i j}^{i}(\mathrm{SO}(2))=\left\{\begin{array}{ll}
\varepsilon_{12}(\mathrm{SO}(2)), & \text { if }\{i, j\}^{\sigma} \in E(\Pi), \\
\iota_{1}(\mathrm{SO}(2)), & \text { if }\{i, j\}^{\sigma} \notin E(\Pi),
\end{array} \quad \phi_{i j}^{j}(\mathrm{SO}(2))= \begin{cases}\varepsilon_{23}(\mathrm{SO}(2)), & \text { if }\{i, j\}^{\sigma} \in E(\Pi), \\
\iota_{2}(\mathrm{SO}(2)), & \text { if }\{i, j\}^{\sigma} \notin E(\Pi) .\end{cases}\right.
$$

The standard SO(2)-amalgam with respect to $\Pi$ and $\sigma$ is the $\mathrm{SO}(2)$-amalgam

$$
\mathcal{A}(\Pi, \sigma, \operatorname{SO}(2)):=\left\{G_{i j}, \phi_{i j}^{i} \mid i \neq j \in I\right\}
$$

with respect to $\Pi$ and $\sigma$ with

$$
\forall i<j \in I: \quad \phi_{i j}^{i}=\left\{\begin{array}{ll}
\varepsilon_{12}, & \text { if }\{i, j\}^{\sigma} \in E(\Pi), \\
\iota_{1}, & \text { if }\{i, j\}^{\sigma} \notin E(\Pi) .
\end{array} \quad \phi_{i j}^{j}= \begin{cases}\varepsilon_{23}, & \text { if }\{i, j\}^{\sigma} \in E(\Pi), \\
\iota_{2}, & \text { if }\{i, j\}^{\sigma} \notin E(\Pi) .\end{cases}\right.
$$

Remark 9.2. The key difference between the standard $\mathrm{SO}(2)$-amalgam and an arbitrary $\mathrm{SO}(2)$-amalgam $\mathcal{A}=\left\{G_{i j}, \phi_{i j}^{i} \mid i \neq j \in I\right\}$ with respect to $\Pi$ and $\sigma$ is that, for instance, $\varepsilon_{12}^{-1} \circ \phi_{i j}^{i}$ can be an arbitrary automorphism of $\mathrm{SO}(2)$. Automatic continuity (like van der Waerden's Continuity Theorem, cf. [HM98, Theorem 5.64 and Corollary 6.56]) fails for automorphisms of the circle group $\mathrm{SO}(2)$ whereas it does hold for the group $\mathrm{SO}(3)$. Hence, obviously, not every automorphism of $\mathrm{SO}(2)$ is induced by an automorphism of $\mathrm{SO}(3)$ and so it is generally not possible to undo the automorphism $\varepsilon_{12}^{-1} \circ \phi_{i j}^{i}$ inside $\mathrm{SO}(3)$. Therefore Goldschmidt's Lemma (see Gol80, Lemma 2.7], also [S02, Proposition 8.3.2], GGH10, Lemma 6.16]) implies that for each diagram $\Pi$ there exist plenty of pairwise non-isomorphic abstract $\mathrm{SO}(2)$-amalgams.

However, by [KP83, Section 4.G], [HKM13, Corollary 7.16], a split real Kac-Moody group and its maximal compact subgroup (i.e., the group of elements fixed by the Cartan-Chevalley involution) both carry natural group topologies that induce the Lie group topology on their respective fundamental subgroups of ranks one and two and make the respective embeddings continuous.

It is therefore meaningful to use continuous $\mathrm{SO}(2)$-amalgams for studying these maximal compact subgroups. Such continuous amalgams are uniquely determined by the underlying diagram $\Pi$, as we will see in Theorem 9.8 below. 
Convention 9.3. For each group isomorphic to one of $\mathrm{SO}(2), \mathrm{SO}(3), \mathrm{SO}(2) \times \mathrm{SO}(2)$, we fix a matrix representation that allows us to identify the respective groups accordingly. Our study of amalgams by Goldschmidt's Lemma then reduces to the study of automorphisms of these groups.

Lemma 9.4. Let

$$
D:=\left(\begin{array}{lll} 
& -1 & 1 \\
1 & &
\end{array}\right) \in \mathrm{SO}(3)
$$

Then the map $\gamma_{D}: \mathrm{SO}(3) \rightarrow \mathrm{SO}(3): A \mapsto D \cdot A \cdot D^{-1}=D \cdot A \cdot D$ is an automorphism of $\mathrm{SO}(3)$ such that

$$
\gamma_{D} \circ \varepsilon_{12}=\varepsilon_{23} \quad \text { and } \quad \gamma_{D} \circ \varepsilon_{23}=\varepsilon_{12} .
$$

Proof. Given $\left(\begin{array}{cc}x & y \\ -y & x\end{array}\right) \in \mathrm{SO}(2)$, we have

$$
\left(\begin{array}{lll} 
& & 1 \\
1 & &
\end{array}\right) \cdot\left(\begin{array}{ccc}
x & y & \\
-y & x & \\
& & 1
\end{array}\right) \cdot\left(\begin{array}{lll} 
& & \\
& -1 & \\
1 & &
\end{array}\right)=\left(\begin{array}{ccc} 
& & 1 \\
y & -x & \\
x & y &
\end{array}\right) \cdot\left(\begin{array}{ccc} 
& & 1 \\
& -1 & \\
1 & &
\end{array}\right)=\left(\begin{array}{ccc}
1 & & \\
& x & y \\
& -y & x
\end{array}\right) .
$$

The second assertion follows analogously.

The only influence of the labelling $\sigma$ of an amalgam is the choice which of the vertices $i^{\sigma}, j^{\sigma}$ corresponds to which subgroup of $G_{i j}$. We now show that this choice does not affect the isomorphism type of the amalgam.

Consequence 9.5. Let $\Pi$ be a simply laced diagram with labellings $\sigma_{1}, \sigma_{2}: I \rightarrow V$. Then

$$
\mathcal{A}\left(\Pi, \sigma_{1}, \mathrm{SO}(2)\right) \cong \mathcal{A}\left(\Pi, \sigma_{2}, \mathrm{SO}(2)\right) .
$$

Proof. Denote $\mathcal{A}:=\mathcal{A}\left(\Pi, \sigma_{1}, \mathrm{SO}(2)\right)$ and $\overline{\mathcal{A}}:=\mathcal{A}\left(\Pi, \sigma_{2}, \mathrm{SO}(2)\right)$. Let $D \in \mathrm{SO}(3)$ be as in Lemma 9.4 and let $\pi:=\sigma_{2}^{-1} \circ \sigma_{1} \in \operatorname{Sym}(I)$. Notice that

$$
\begin{aligned}
\bar{G}_{\pi(i) \pi(j)}=\mathrm{SO}(3) & \Leftrightarrow\{\pi(i), \pi(j)\}^{\sigma_{2}} \in E(\Pi) \Leftrightarrow\{i, j\}^{\pi \sigma_{2}} \in E(\Pi) \\
& \Leftrightarrow\{i, j\}^{\sigma_{1} \sigma_{2}^{-1} \sigma_{2}} \in E(\Pi) \Leftrightarrow\{i, j\}^{\sigma_{1}} \in E(\Pi) \Leftrightarrow G_{i j}=\mathrm{SO}(3) . .
\end{aligned}
$$

Given $i<j \in I$ with $\{i, j\}^{\sigma_{1}} \in E(\Pi)$, let

$$
\alpha_{i j}:= \begin{cases}\operatorname{id}_{\mathrm{SO}(3)}, & \text { if } \pi(i)<\pi(j), \\ \gamma_{D}, & \text { if } \pi(i)>\pi(j),\end{cases}
$$

and given $i<j \in I$ with $\{i, j\}^{\sigma_{1}} \notin E(\Pi)$, let

$$
\alpha_{i j}: \mathrm{SO}(2) \times \mathrm{SO}(2) \rightarrow \mathrm{SO}(2) \times \mathrm{SO}(2),(x, y) \mapsto \begin{cases}(x, y), & \text { if } \pi(i)<\pi(j), \\ (y, x), & \text { if } \pi(i)>\pi(j) .\end{cases}
$$

Then the system $\alpha:=\left\{\pi, \alpha_{i j} \mid i \neq j \in I\right\}: \mathcal{A} \rightarrow \overline{\mathcal{A}}$ is an isomorphism of amalgams. Indeed, given $i<j \in I$ with $\{i, j\}^{\sigma_{1}} \in E(\Pi)$, one has

$$
\alpha_{i j} \circ \phi_{i j}^{i}=\alpha_{i j} \circ \varepsilon_{12}= \begin{cases}\operatorname{id}_{\mathrm{SO}(3)} \circ \varepsilon_{12}=\varepsilon_{12}=\bar{\phi}_{\pi(i) \pi(j)}^{\pi(i)}, & \text { if } \pi(i)<\pi(j), \\ \gamma_{D} \circ \varepsilon_{12}=\varepsilon_{23}=\bar{\phi}_{\pi(i) \pi(j)}^{\pi(j)}, & \text { if } \pi(i)>\pi(j),\end{cases}
$$

and

$$
\alpha_{i j} \circ \phi_{i j}^{j}=\alpha_{i j} \circ \varepsilon_{23}= \begin{cases}\operatorname{id}_{\mathrm{SO}(3)} \circ \varepsilon_{23}=\varepsilon_{23}=\bar{\phi}_{\pi(i) \pi(j)}^{\pi(i)}, & \text { if } \pi(i)<\pi(j), \\ \gamma_{D} \circ \varepsilon_{23}=\varepsilon_{12}=\bar{\phi}_{\pi(i) \pi(j)}^{\pi(j)}, & \text { if } \pi(i)>\pi(j) .\end{cases}
$$

The case $i<j \in I$ with $\{i, j\}^{\sigma_{1}} \notin E(\Pi)$ is verified similarly.

Definition 9.6. As we have just seen, the labelling of a standard $\mathrm{SO}(2)$-amalgam is irrelevant for its isomorphism type. Hence, for a simply laced diagram $\Pi$, we write $\mathcal{A}(\Pi, \mathrm{SO}(2))$ to denote this isomorphism type and, moreover, by slight abuse of notation to denote any representative $\mathcal{A}(\Pi, \sigma, \mathrm{SO}(2))$ of this isomorphism type. It is called the standard $\mathrm{SO}(2)$-amalgam with respect to $\Pi$.

Lemma 9.7. Let $B:=\left({ }^{1}-I_{2}\right), C:=\left(\begin{array}{ll}{ }^{-I_{2}} & \\ & \end{array}\right) \in \mathrm{SO}(3)$. Then the following holds:

(a) The map $\gamma_{B}: \mathrm{SO}(3) \rightarrow \mathrm{SO}(3): A \mapsto B \cdot A \cdot B^{-1}$ is an automorphism of $\mathrm{SO}(3)$ such that

$$
\gamma_{B} \circ \varepsilon_{12}=\varepsilon_{12} \circ \text { inv } \quad \underset{17}{\text { and }} \quad \gamma_{B} \circ \varepsilon_{23}=\varepsilon_{23} .
$$


(b) The map $\gamma_{C}: \mathrm{SO}(3) \rightarrow \mathrm{SO}(3): A \mapsto C \cdot A \cdot C^{-1}$ is an automorphism of $\mathrm{SO}(3)$ such that

$$
\gamma_{C} \circ \varepsilon_{12}=\varepsilon_{12} \quad \text { and } \quad \gamma_{C} \circ \varepsilon_{23}=\varepsilon_{23} \circ \text { inv. }
$$

Proof. (a) Given $A:=\left(\begin{array}{cc}x & y \\ -y & x\end{array}\right) \in \mathrm{SO}(2)$, we have

$$
\gamma_{B}\left(\varepsilon_{12}(A)\right)=B \cdot\left(\begin{array}{ccc}
x & y & \\
-y & x & \\
& & 1
\end{array}\right) \cdot B^{-1}=\left(\begin{array}{ccc}
x & -y & \\
y & x & \\
& & 1
\end{array}\right)=\varepsilon_{12}\left(A^{-1}\right) .
$$

The second statement follows analogously.

(b) is shown with a similar computation.

Theorem 9.8. Let $\Pi$ be a simply laced diagram with labelling $\sigma: I \rightarrow V$ and let $\mathcal{A}=\left\{G_{i j}, \phi_{i j}^{i} \mid i \neq j \in I\right\}$ be a continuous $\mathrm{SO}(2)$-amalgam with respect to $\Pi$ and $\sigma$. Then $\mathcal{A} \cong \mathcal{A}(\Pi, \sigma, \mathrm{SO}(2))$.

Proof. Denote $\overline{\mathcal{A}}:=\mathcal{A}(\Pi, \sigma, \mathrm{SO}(2))$. The only continuous automorphisms of the circle group $\mathrm{SO}(2)$ are id and the inversion inv. Since $\mathcal{A}$ is continuous by hypothesis, for all $i<j \in I$ with $\{i, j\}^{\sigma} \in E(\Pi)$ we have

$$
\phi_{i j}^{i} \in\left\{\varepsilon_{12}, \varepsilon_{12} \circ \text { inv }\right\}, \quad \phi_{i j}^{j} \in\left\{\varepsilon_{23}, \varepsilon_{23} \circ \text { inv }\right\} .
$$

Let $B, C \in \mathrm{SO}(3)$ be as in Lemma 9.7 let $\pi:=\operatorname{id}_{I}$, and given $i>j \in I$ such that $\{i, j\}^{\sigma} \in E(\Pi)$, let

$$
\alpha_{i j}:=\left\{\begin{array}{lll}
\operatorname{id}_{\mathrm{SO}(3)}, & \text { if } \phi_{i j}^{i}=\varepsilon_{12}, & \phi_{i j}^{j}=\varepsilon_{23}, \\
\gamma_{B}, & \text { if } \phi_{i j}^{i}=\varepsilon_{12} \circ \text { inv }, & \phi_{i j}^{j}=\varepsilon_{23}, \\
\gamma_{C}, & \text { if } \phi_{i j}^{i}=\varepsilon_{12}, & \phi_{i j}^{j}=\varepsilon_{23} \circ \text { inv }, \\
\gamma_{B} \circ \gamma_{C}, & \text { if } \phi_{i j}^{i}=\varepsilon_{12} \circ \text { inv }, & \phi_{i j}^{j}=\varepsilon_{23} \circ \text { inv. }
\end{array}\right.
$$

For $i<j \in I$ with $\{i, j\}^{\sigma} \notin E(\Pi)$, define $\alpha_{i j}:=\left(\iota_{1} \circ\left(\phi_{i j}^{i}\right)^{-1}\right) \times\left(\iota_{2} \circ\left(\phi_{i j}^{j}\right)^{-1}\right)$. Then the system $\alpha:=\left\{\pi, \alpha_{i j} \mid i \neq j \in I\right\}: \mathcal{A} \rightarrow \overline{\mathcal{A}}$ is an isomorphism of amalgams.

The following is well-known, e.g. DMGH09, Theorem 1.2].

Theorem 9.9. For $n \geq 3$, the group $\mathrm{SO}(n)$ is a universal enveloping group of $\mathcal{A}\left(A_{n-1}, \mathrm{SO}(2)\right)$.

Proof. Let $I:=\{1, \ldots, n-1\}$. The group $\mathrm{SO}(n)$ acts flag-transitively on the simply connected projective geometry $\mathcal{G}:=\mathbb{P}_{n-1}(\mathbb{R})$; simple connectedness follows from [Tit74, Theorem 13.32], Tit81, Theorem 2], [BC13, Proposition 11.1.9, Theorem 11.1.13], flag-transitivity of the action from the Iwasawa/QRdecomposition of $\mathrm{SL}_{n}(\mathbb{R})$. A maximal flag is given by

$$
\left\langle e_{1}\right\rangle_{\mathbb{R}} \leq\left\langle e_{1}, e_{2}\right\rangle_{\mathbb{R}} \leq \cdots \leq\left\langle e_{1}, \ldots, e_{n-1}\right\rangle_{\mathbb{R}}
$$

Let $T$ be the subgroup of $\mathrm{SO}(n)$ of diagonal matrices; it is isomorphic to $C_{2}^{n-1}$ (where $C_{2}$ is a cyclic group of order 2). Moreover, for $1 \leq i \leq n-1$, let $H_{i} \cong \mathrm{SO}(2)$ be the circle group acting naturally on $\left\langle e_{i}, e_{i+1}\right\rangle_{\mathbb{R}}$ and, for $1 \leq i \leq n-2$, let $\bar{H}_{i, i+1} \cong \mathrm{SO}(3)$ be the group acting naturally on $\left\langle e_{i}, e_{i+1}, e_{i+2}\right\rangle_{\mathbb{R}}$. Furthermore, for $1 \leq i<j-1 \leq n-2$, let $H_{i j}:=H_{i} H_{j} \cong \mathrm{SO}(2) \times \mathrm{SO}(2)$. Then the stabilizer of a sub-flag of co-rank one is of the form $H_{i} T, 1 \leq i \leq n-1$, and the stabilizer of a sub-flag of co-rank two is of the form $H_{i j} T, 1 \leq i<j \leq n-1$.

The group $T \cong C_{2}^{n-1}$ admits a presentation with all generators and relations contained in the rank two subgroups $H_{i j}$ of $\mathrm{SO}(n)$ : Indeed, $T$ is generated by the groups $C_{2} \cong T_{i}:=\langle-1\rangle \leq H_{i} \cong \mathrm{SO}(2)$ and for each $1 \leq i \neq j \leq n-1$ the relation $T_{i} T_{j}=T_{j} T_{i}$ is visible within $H_{i j}$. Therefore, by an iteration of Tits's Lemma (see [Tit86, Corollary 1], IS02, Corollary 1.4.6]) with respect to the above maximal flag, the group $H:=\mathrm{SO}(n)$ is the universal enveloping group of the amalgam $\mathcal{A}(\mathcal{G}, H)=\left\{H_{i j}, \Phi_{i j}^{i} \mid i \neq j \in I\right\}$, where $\Phi_{i j}^{i}: H_{i} \rightarrow H_{i j}$ is the inclusion map for each $i \neq j \in I$. One has

$$
\forall i \in I: \quad H_{i}=\varepsilon_{\{i, i+1\}}(\mathrm{SO}(2))
$$

and

$$
\forall i<j \in I: \quad H_{i j}= \begin{cases}\varepsilon_{\{i, i+1, i+2\}}(\mathrm{SO}(3)), & \text { if } j=i+1, \\ \varepsilon_{\{i, i+1\}}(\mathrm{SO}(2)) \times \varepsilon_{\{j, j+1\}}(\mathrm{SO}(2)), & \text { if } j \neq i+1 .\end{cases}
$$

As a consequence, the system

$$
\alpha=\left\{\operatorname{id}_{I}, \alpha_{i j}, \alpha_{i} \mid i \neq j \in I\right\}: \mathcal{A}\left(A_{n-1}, \mathrm{SO}(2)\right) \rightarrow \mathcal{A}(\mathcal{G}, H)
$$

with

$$
\forall i \in I: \quad \alpha_{i}=\varepsilon_{\{i, i+1\}}: \mathrm{SO}(2) \rightarrow H_{i}
$$


and

$$
\forall i<j \in I: \quad \alpha_{i j}= \begin{cases}\varepsilon_{\{i, i+1, i+2\}}, & \text { if } j=i+1, \\ \varepsilon_{\{i, i+1\}} \times \varepsilon_{\{j, j+1\}}, & \text { if } j \neq i+1,\end{cases}
$$

is an isomorphism of amalgams.

Remark 9.10. The above proof mainly relies on geometric arguments in the Tits building of type $A_{n-1}$. We exploit this to generalize the above statements to other diagrams, see Theorems 11.2 and 16.15. The crucial observation to make is that — via the local-to-global principle — it basically suffices to understand the rank two situation in order to understand arbitrary types.

\section{Spin(2)-AMALGAMS OF SIMPLY LACED TYPE}

In analogy to Section 9 we now study the amalgamation of groups isomorphic to Spin(3), continuously glued to one another along circle groups. In particular, we describe $\operatorname{Spin}(n)$ as the universal enveloping group of its Spin(2)-amalgam and relate the classification of continuous Spin(2)-amalgams to the classification of continuous $\mathrm{SO}(2)$-amalgams via the lifting of automorphisms.

Recall the maps $\tilde{\varepsilon}_{12}, \tilde{\varepsilon}_{23}: \operatorname{Spin}(2) \rightarrow \operatorname{Spin}(3)$ from Lemma 6.10 and the maps $\tilde{\iota}_{1}, \tilde{\iota}_{2}: \operatorname{Spin}(2) \rightarrow$ $\operatorname{Spin}(2) \times \operatorname{Spin}(2)$ from Notation 8.1

Definition 10.1. Let $\Pi$ be a simply laced diagram with labelling $\sigma: I \rightarrow V$. A Spin(2)-amalgam with respect to $\Pi$ and $\sigma$ is an amalgam $\mathcal{A}=\left\{G_{i j}, \phi_{i j}^{i} \mid i \neq j \in I\right\}$ such that

$$
\forall i \neq j \in I: \quad G_{i j}= \begin{cases}\operatorname{Spin}(3), & \text { if }\{i, j\}^{\sigma} \in E(\Pi), \\ \operatorname{Spin}(2) \times \operatorname{Spin}(2), & \text { if }\{i, j\}^{\sigma} \notin E(\Pi),\end{cases}
$$

and for $i<j \in I$,

$$
\phi_{i j}^{i}(\operatorname{Spin}(2))=\left\{\begin{array}{ll}
\tilde{\varepsilon}_{12}(\operatorname{Spin}(2)), & \text { if }\{i, j\}^{\sigma} \in E(\Pi), \\
\iota_{1}(\operatorname{Spin}(2)), & \text { if }\{i, j\}^{\sigma} \notin E(\Pi),
\end{array} \phi_{i j}^{j}(\operatorname{Spin}(2))= \begin{cases}\tilde{\varepsilon}_{23}(\operatorname{Spin}(2)), & \text { if }\{i, j\}^{\sigma} \in E(\Pi), \\
\iota_{2}(\operatorname{Sppin}(2)), & \text { if }\{i, j\}^{\sigma} \notin E(\Pi) .\end{cases}\right.
$$

The standard Spin(2)-amalgam with respect to $\Pi$ and $\boldsymbol{\sigma}$ is the (continuous) Spin(2)-amalgam

$$
\mathcal{A}(\Pi, \sigma, \operatorname{Spin}(2)):=\left\{G_{i j}, \phi_{i j}^{i}, \mid i \neq j \in I\right\}
$$

with respect to $\Pi$ and $\sigma$ with

$$
\forall i<j \in I: \quad \phi_{i j}^{i}=\left\{\begin{array}{ll}
\tilde{\varepsilon}_{12}, & \text { if }\{i, j\}^{\sigma} \in E(\Pi), \\
\tilde{\iota}_{1}, & \text { if }\{i, j\}^{\sigma} \notin E(\Pi) .
\end{array} \quad \phi_{i j}^{j}= \begin{cases}\tilde{\varepsilon}_{23}, & \text { if }\{i, j\}^{\sigma} \in E(\Pi), \\
\tilde{\iota}_{2}, & \text { if }\{i, j\}^{\sigma} \notin E(\Pi) .\end{cases}\right.
$$

Notation 10.2. Proposition 8.2 enables us to lift $\mathrm{SO}(2)$-amalgams to $\operatorname{Spin}(2)$-amalgams: Let $\Pi$ be a simply laced diagram with labelling $\sigma: I \rightarrow V$ and let $\mathcal{A}=\left\{G_{i j}, \phi_{i j}^{i} \mid i \neq j \in I\right\}$ be an $\operatorname{SO}(2)$-amalgam with respect to $\Pi$ and $\sigma$. Given $i>j \in I$, there are $\gamma_{i j}^{i}, \gamma_{i j}^{j} \in \operatorname{Aut}(\operatorname{SO}(2))$ such that

$$
\phi_{i j}^{i}=\left\{\begin{array}{ll}
\varepsilon_{12} \circ \gamma_{i j}^{j}, & \text { if }\{i, j\}^{\sigma} \in E(\Pi), \\
\iota_{1} \circ \gamma_{i j}^{j}, & \text { if }\{i, j\}^{\sigma} \notin E(\Pi),
\end{array} \quad \phi_{i j}^{j}= \begin{cases}\varepsilon_{23} \circ \gamma_{i j}^{j}, & \text { if }\{i, j\}^{\sigma} \in E(\Pi), \\
\iota_{2} \circ \gamma_{i j}^{j}, & \text { if }\{i, j\}^{\sigma} \notin E(\Pi) .\end{cases}\right.
$$

We then lift $\gamma_{i j}^{i}, \gamma_{i j}^{j}$ as in Lemma 8.2 to $\tilde{\gamma}_{i j}^{i}, \tilde{\gamma}_{i j}^{j} \in \operatorname{Aut}(\operatorname{Spin}(2))$ and set

$$
\tilde{\phi}_{i j}^{i}:=\left\{\begin{array}{ll}
\tilde{\varepsilon}_{12} \circ \tilde{\gamma}_{i j}^{i}, & \text { if }\{i, j\}^{\sigma} \in E(\Pi), \\
\tilde{\iota}_{1} \circ \tilde{\gamma}_{i j}^{i}, & \text { if }\{i, j\}^{\sigma} \notin E(\Pi),
\end{array} \quad \tilde{\phi}_{i j}^{j}:= \begin{cases}\tilde{\varepsilon}_{23} \circ \tilde{\gamma}_{i j}^{j}, & \text { if }\{i, j\}^{\sigma} \in E(\Pi), \\
\tilde{\iota}_{2} \circ \tilde{\gamma}_{i j}^{j}, & \text { if }\{i, j\}^{\sigma} \notin E(\Pi),\end{cases}\right.
$$

and

$$
\widetilde{G}_{i j}:= \begin{cases}\operatorname{Spin}(3), & \text { if }\{i, j\}^{\sigma} \in E(\Pi) \\ \operatorname{Spin}(2) \times \operatorname{Spin}(2), & \text { if }\{i, j\}^{\sigma} \notin E(\Pi)\end{cases}
$$

Definition 10.3. Let $\Pi$ be a simply laced diagram with labelling $\sigma: I \rightarrow V$ and let $\mathcal{A}=\left\{G_{i j}, \phi_{i j}^{i} \mid i \neq\right.$ $j \in I\}$ be an $\operatorname{SO}(2)$-amalgam with respect to $\Pi$ and $\sigma$. Then $\widetilde{\mathcal{A}}:=\left\{\widetilde{G}_{i j}, \tilde{\phi}_{i j}^{i} \mid i \neq j \in I\right\}$ is the induced $\operatorname{Spin}(2)$-amalgam with respect to $\Pi$ and $\sigma$. We also set

$$
\rho_{i j}:= \begin{cases}\rho_{3}, & \text { if }\{i, j\}^{\sigma} \in E(\Pi), \\ \rho_{2} \times \rho_{2}, & \text { if }\{i, j\}^{\sigma} \notin E(\Pi) .\end{cases}
$$


Lemma 10.4. Let $\Pi$ be a simply laced diagram with labelling $\sigma: I \rightarrow V$, let $\mathcal{A}=\left\{G_{i j}, \phi_{i j}^{i} \mid i \neq j \in I\right\}$ be an $\mathrm{SO}(2)$-amalgam with respect to $\Pi$ and $\sigma$, and let $\tilde{\phi}_{i j}^{i}$ be as introduced in Notation 10.2. Then for all $i \neq j \in I$

$$
\phi_{i j}^{i} \circ \rho_{2}=\rho_{i j} \circ \tilde{\phi}_{i j}^{i}
$$

Proof. Without loss of generality suppose $i<j \in I$. Let $\gamma_{i j}^{i}, \gamma_{i j}^{j} \in \operatorname{Aut}(\operatorname{SO}(2)), \tilde{\gamma}_{i j}^{i}, \tilde{\gamma}_{i j}^{j} \in \operatorname{Aut}(\operatorname{Spin}(2))$, and $\tilde{\phi}_{i j}^{i}, \tilde{\phi}_{i j}^{j}$ be as in Notation 10.2. Then, if $\{i, j\}^{\sigma} \in E(\Pi)$, we find that

$$
\phi_{i j}^{i} \circ \rho_{2}=\varepsilon_{12} \circ \gamma_{i j}^{i} \circ \rho_{2}=\varepsilon_{12} \circ \rho_{2} \circ \tilde{\gamma}_{i j}^{i} \stackrel{6.13}{=} \rho_{3} \circ \varepsilon_{12} \circ \tilde{\gamma}_{i j}^{i}=\rho_{3} \circ \tilde{\phi}_{i j}^{i} .
$$

Similarly we also conclude $\phi_{i j}^{j} \circ \rho_{2}=\rho_{3} \circ \tilde{\phi}_{i j}^{j}$. Moreover, in case $\{i, j\}^{\sigma} \notin E(\Pi)$ we deduce

$$
\phi_{i j}^{i} \circ \rho_{2}=\varepsilon_{12} \circ \gamma_{i j}^{i} \circ \rho_{2}=\varepsilon_{12} \circ \rho_{2} \circ \tilde{\gamma}_{i j}^{i}=\left(\rho_{2} \times \rho_{2}\right) \circ \tilde{\iota}_{1} \circ \tilde{\gamma}_{i j}^{i}=\left(\rho_{2} \times \rho_{2}\right) \circ \tilde{\phi}_{i j}^{i} .
$$

Again we conclude by a similar argument that also $\phi_{i j}^{j} \circ \rho_{2}=\left(\rho_{2} \times \rho_{2}\right) \circ \tilde{\phi}_{i j}^{j}$.

Remark 10.5. Clearly the construction of an induced Spin(2)-amalgam is symmetric and can also be applied backwards: Starting with a $\operatorname{Spin}(2)$-amalgam $\hat{\mathcal{A}}$ one can construct an $\mathrm{SO}(2)$-amalgam $\mathcal{A}$, such that $\hat{\mathcal{A}}=\widetilde{\mathcal{A}}$. In particular, we obtain an epimorphism for the standard $\operatorname{Spin}(2)$ - and $\mathrm{SO}(2)$-amalgams with respect to $\Pi$ and $\sigma$, which we denote by

$$
\pi_{\Pi, \sigma}=\left\{\operatorname{id}_{I}, \rho_{2}, \rho_{i j}\right\}: \mathcal{A}(\Pi, \sigma, \operatorname{Spin}(2)) \rightarrow \mathcal{A}(\Pi, \sigma, \mathrm{SO}(2)) .
$$

Proposition 10.6. Let $\Pi$ be a simply laced diagram with labelling $\sigma: I \rightarrow V$, let $\mathcal{A}_{1}$ and $\mathcal{A}_{2}$ be $\mathrm{SO}(2)$ amalgams with respect to $\Pi$ and $\sigma$, and let $\alpha=\left\{\pi, \alpha_{i j} \mid i \neq j \in I\right\}: \mathcal{A}_{1} \rightarrow \mathcal{A}_{2}$ be an isomorphism of amalgams. Then there is a unique isomorphism $\tilde{\alpha}=\left\{\pi, \tilde{\alpha}_{i j} \mid i \neq j \in I\right\}: \widetilde{\mathcal{A}}_{1} \rightarrow \widetilde{\mathcal{A}}_{2}$ such that for all $i \neq j \in I$

$$
\rho_{\pi(i) \pi(j)} \circ \tilde{\alpha}_{i j}=\alpha_{i j} \circ \rho_{i j}
$$

Proof. Suppose $\mathcal{A}_{1}=\left\{G_{i j}, \phi_{i j}^{i} \mid i \neq j \in I\right\}$ and $\mathcal{A}_{2}=\left\{H_{i j}, \psi_{i j}^{i} \mid i \neq j \in I\right\}$. Let $i \neq j \in I$. Since both amalgams are defined with respect to $\Pi$ and $\sigma$, and since $G_{i j} \cong H_{\pi(i) \pi(j)}$, we conclude that $\{i, j\}^{\sigma}$ is an edge if and only if $\{i, j\}^{\pi \sigma}$ is an edge. Hence up to relabeling and identifying $G_{i j}$ with its image under $\alpha_{i j}$, we may assume $\pi=\mathrm{id}$ and $G_{i j}=H_{i j}$ and, thus, $\alpha_{i j} \in \operatorname{Aut}\left(G_{i j}\right)$ with $\alpha_{i j} \circ \phi_{i j}^{i}=\psi_{i j}^{i}$. We distinguish two cases.

Case I: $\{\boldsymbol{i}, \boldsymbol{j}\}^{\boldsymbol{\sigma}} \in \boldsymbol{E}(\boldsymbol{\Pi})$ : Then $\widetilde{G}_{i j}=\operatorname{Spin}(3)$. Let $\tilde{\alpha}_{i j} \in \operatorname{Aut}(\operatorname{Spin}(3))$ be the unique automorphism from Proposition 8.4 satisfying $\rho_{3} \circ \tilde{\alpha}_{i j}=\alpha_{i j} \circ \rho_{3}$. It remains to verify that this is compatible with the amalgam structure. Indeed,

$$
\begin{gathered}
\rho_{3} \circ \tilde{\alpha}_{i j} \circ \tilde{\phi}_{i j}^{i} \circ\left(\tilde{\psi}_{i j}^{i}\right)^{-1}=\alpha_{i j} \circ \rho_{3} \circ \tilde{\phi}_{i j}^{i} \circ\left(\tilde{\psi}_{i j}^{i}\right)^{-1} \stackrel{10.4}{=} \alpha_{i j} \circ \phi_{i j}^{i} \circ \rho_{2} \circ\left(\psi_{i j}^{i}\right)^{-1} \\
\stackrel{10.4}{\underline{=}} \underbrace{\alpha_{i j} \circ \phi_{i j}^{i} \circ\left(\psi_{i j}^{i}\right)^{-1}}_{=\operatorname{id}{ }_{\text {SO }(3)}} \circ \rho_{3}=\rho_{3}=\rho_{3} \circ \operatorname{id}_{\operatorname{Spin}(3)} .
\end{gathered}
$$

Hence, by uniqueness in Proposition 8.4 one has $\tilde{\alpha}_{i j} \circ \tilde{\phi}_{i j}^{i} \circ\left(\tilde{\psi}_{i j}^{i}\right)^{-1}=\mathrm{id}_{\operatorname{Spin}(3)}$, i.e., $\tilde{\alpha}_{i j} \circ \tilde{\phi}_{i j}^{i}=\tilde{\psi}_{i j}^{i}$. Case II: $\{\boldsymbol{i}, \boldsymbol{j}\}^{\boldsymbol{\sigma}} \notin \boldsymbol{E}(\boldsymbol{\Pi})$ : Then $\widetilde{G}_{i j}=\operatorname{Spin}(2) \times \operatorname{Spin}(2)$. Let $\tilde{\alpha}_{i j} \in \operatorname{Aut}(\operatorname{Spin}(2) \times \operatorname{Spin}(2))$ be the unique automorphism from Corollary 8.3 satisfying $\left(\rho_{2} \times \rho_{2}\right) \circ \tilde{\alpha}_{i j}=\alpha_{i j} \circ\left(\rho_{2} \times \rho_{2}\right)$. It remains to verify that this is compatible with the amalgam structure. Indeed,

$$
\begin{aligned}
& \left(\rho_{2} \times \rho_{2}\right) \circ \tilde{\alpha}_{i j} \circ \tilde{\phi}_{i j}^{i} \circ\left(\tilde{\psi}_{i j}^{i}\right)^{-1}=\alpha_{i j} \circ\left(\rho_{2} \times \rho_{2}\right) \circ \tilde{\phi}_{i j}^{i} \circ\left(\tilde{\psi}_{i j}^{i}\right)^{-1} \underline{\underline{\underline{10.4}}} \alpha_{i j} \circ \phi_{i j}^{i} \circ \rho_{2} \circ\left(\psi_{i j}^{i}\right)^{-1} \\
& \stackrel{10.4}{\underline{\underline{1}}} \underbrace{\alpha_{i j} \circ \phi_{i j}^{i} \circ\left(\psi_{i j}^{i}\right)^{-1}}_{=\mathrm{id}_{\mathrm{SO}(2) \times \mathrm{SO}(2)}} \circ\left(\rho_{2} \times \rho_{2}\right)=\left(\rho_{2} \times \rho_{2}\right) . .
\end{aligned}
$$

By uniqueness in Corollary 8.3 , one concludes as in the previous case that $\tilde{\alpha}_{i j} \circ \tilde{\phi}_{i j}^{i}=\tilde{\psi}_{i j}^{i}$.

Corollary 10.7. Let $\Pi$ be a simply laced diagram with labellings $\sigma_{1}, \sigma_{2}$. Then $\mathcal{A}\left(\Pi, \sigma_{1}, \operatorname{Spin}(2)\right) \cong$ $\mathcal{A}\left(\Pi, \sigma_{2}, \operatorname{Spin}(2)\right)$.

Proof. Let $\mathcal{A}_{1}:=\mathcal{A}\left(\Pi, \sigma_{1}, \mathrm{SO}(2)\right)$ and $\mathcal{A}_{2}:=\mathcal{A}\left(\Pi, \sigma_{2}, \mathrm{SO}(2)\right)$. The definitions imply $\widetilde{\mathcal{A}}_{1}=\mathcal{A}\left(\Pi, \sigma_{1}, \operatorname{Spin}(2)\right)$ and $\widetilde{\mathcal{A}}_{2}=\mathcal{A}\left(\Pi, \sigma_{2}\right.$, $\left.\operatorname{Spin}(2)\right)$. Moreover, one has $\mathcal{A}_{1} \cong \mathcal{A}_{2}$ by Consequence 9.5. The claim now follows by applying Proposition 10.6 . 
Definition 10.8. As before, for a simply laced diagram $\Pi$ with labelling $\sigma$, we write $\mathcal{A}(\Pi, \operatorname{Spin}(2))$ to denote the isomorphism type of $\mathcal{A}(\Pi, \sigma$, $\operatorname{Spin}(2))$ and, by slight abuse of notation, any representative of this isomorphism type. It is called the standard $\operatorname{Spin}(2)$-amalgam with respect to $\Pi$.

Theorem 10.9. Let $\Pi$ be a simply laced diagram with labelling $\sigma: I \rightarrow V$ and let $\widetilde{\mathcal{A}}$ be a continuous $\operatorname{Spin}(2)$-amalgam with respect to $\Pi$ and $\sigma$. Then $\widetilde{\mathcal{A}} \cong \mathcal{A}(\Pi, \operatorname{Spin}(2))$.

Proof. Let $\mathcal{A}$ be the continuous $\mathrm{SO}(2)$-amalgam that induces $\widetilde{\mathcal{A}}$, which exists by Remark 10.5 . By Theorem 9.8, one has $\mathcal{A} \cong \mathcal{A}(\Pi, \mathrm{SO}(2))$. Proposition 10.6 yields the claim, since $\mathcal{A}(\Pi, \mathrm{SO}(2))$ induces $\mathcal{A}(\Pi, \operatorname{Spin}(2))$.

Theorem 10.10. For $n \geq 3$, the group $\operatorname{Spin}(n)$ is the universal enveloping group of $\mathcal{A}\left(A_{n-1}, \operatorname{Spin}(2)\right)$.

Proof. The proof runs along the same lines as the proof of Theorem 9.9. Let $I:=\{1, \ldots, n-1\}$. The group $\operatorname{Spin}(n)$ acts flag-transitively via the twisted adjoint representation (cf. Theorem $6.8(\mathrm{~b})$ ) on the simply connected projective geometry $\mathcal{G}:=\mathbb{P}_{n-1}(\mathbb{R})$ with fundamental maximal flag $\left\langle e_{1}\right\rangle_{\mathbb{R}} \leq\left\langle e_{1}, e_{2}\right\rangle_{\mathbb{R}} \leq$ $\cdots \leq\left\langle e_{1}, \ldots, e_{n-1}\right\rangle_{\mathbb{R}}$. By an iteration of Tits's Lemma (Tit86, Corollary 1], IS02, Corollary 1.4.6]) with respect to the above maximal flag, the group $H:=\operatorname{Spin}(n)$ is the universal enveloping group of the amalgam

$$
\mathcal{A}(\mathcal{G}, H)=\left\{H_{i j}, \phi_{i j}^{i} \mid i \neq j \in I\right\},
$$

where the $H_{i j}$ are the "block-diagonal" rank two subgroups and $\Phi_{i j}^{i}: H_{i} \rightarrow H_{i j}$ is the inclusion map for each $i \neq j \in I$. By Consequence 6.12 and Remark 6.13, one has

$$
\forall i \in I: \quad H_{i}=\tilde{\varepsilon}_{\{i, i+1\}}(\operatorname{Spin}(2))
$$

and

$$
\forall i<j \in I: \quad H_{i j}= \begin{cases}\tilde{\varepsilon}_{\{i, i+1, i+2\}}(\operatorname{Spin}(3)), & \text { if } j=i+1, \\ \tilde{\varepsilon}_{\{i, i+1\}}(\operatorname{Spin}(2)) \cdot \tilde{\varepsilon}_{\{j, j+1\}}(\operatorname{Spin}(2)), & \text { if } j \neq i+1 .\end{cases}
$$

As a consequence, the system

$$
\alpha=\left\{\operatorname{id}_{I}, \alpha_{i j}, \alpha_{i} \mid i \neq j \in I\right\}: \mathcal{A}\left(A_{n-1}, \operatorname{Spin}(2)\right) \rightarrow \mathcal{A}(\mathcal{G}, H)
$$

with

and

$$
\forall i \in I: \quad \alpha_{i}=\tilde{\varepsilon}_{\{i, i+1\}}: \operatorname{Spin}(2) \rightarrow H_{i}
$$

$$
\forall i<j \in I: \quad \alpha_{i j}= \begin{cases}\tilde{\varepsilon}_{\{i, i+1, i+2\}}, & \text { if } j=i+1, \\ \varepsilon_{\{i, i+1\}} \cdot \tilde{\varepsilon}_{\{j, j+1\}}, & \text { if } j \neq i+1,\end{cases}
$$

is an epimorphism of $\operatorname{Spin}(2)$-amalgams. In fact, each $\alpha_{i}$ and each $\alpha_{i i+1}$ is an isomorphism, only the $\alpha_{i j}: \operatorname{Spin}(2) \times \operatorname{Spin}(2) \rightarrow \operatorname{Spin}(2) \cdot \operatorname{Spin}(2), j \neq i+1$, have a kernel of order two: the -1 in the left-hand factor gets identified with the -1 in the right-hand factor. We may conclude that $\operatorname{Spin}(n)$ is an enveloping group of $\mathcal{A}\left(A_{n-1}, \operatorname{Spin}(2)\right)$. It remains to prove universality.

Let $\left(G, \tau_{i j}\right)$ be an arbitrary enveloping group of $\mathcal{A}\left(A_{n-1}\right.$, $\left.\operatorname{Spin}(2)\right)$ and let $1 \leq i<j \leq n-1$ with $j \neq i+1$. By definition the following diagram commutes for $1 \leq a \neq b \neq c \leq n-1$ :

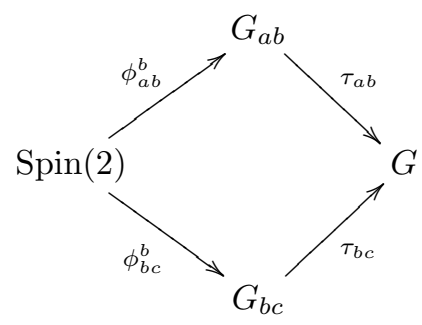

In particular, one has:

$$
\begin{aligned}
\left(\tau_{i, i+2} \circ \phi_{i, i+2}^{i}\right)(-1) & =\left(\tau_{i, i+1} \circ \phi_{i, i+1}^{i}\right)(-1) \\
& =\left(\tau_{i, i+1} \circ \phi_{i, i+1}^{i+1}\right)(-1) \\
& =\left(\tau_{i+1, i+2} \circ \phi_{i+1, i+2}^{i+1}\right)(-1) \\
& =\left(\tau_{i+1, i+2} \circ \phi_{i+1, i+2}^{i+2}\right)(-1) \\
& =\left(\tau_{i, i+2} \circ \phi_{i, i+2}^{i+2}\right)(-1)
\end{aligned}
$$$$
\begin{array}{r}
(\text { set } b=i, a=i+1, c=i+2) \\
\left(\text { since } \tilde{\varepsilon}_{12}(-1)=\tilde{\varepsilon}_{23}(-1)\right) \\
(\text { set } b=i+1, a=i, c=i+2) \\
\left(\text { since } \tilde{\varepsilon}_{12}(-1)=\tilde{\varepsilon}_{23}(-1)\right) \\
(\text { set } b=i+2, a=i, c=i+1) .
\end{array}
$$ 
We conclude by induction that $\tau_{i j}: \operatorname{Spin}(2) \times \operatorname{Spin}(2) \rightarrow G, j \neq i+1$, always factors through $\operatorname{Spin}(2) . \operatorname{Spin}(2)$ or, in other words, $\tau: \mathcal{A}\left(A_{n-1}\right.$, $\left.\operatorname{Spin}(2)\right) \rightarrow G$ always factors through $\mathcal{A}(\mathcal{G}, H)$. That is, the universal enveloping group $\operatorname{Spin}(n)$ of $\mathcal{A}(\mathcal{G}, H)$ is also a universal enveloping group of $\mathcal{A}\left(A_{n-1}, \operatorname{Spin}(2)\right)$.

Remark 10.11. The proof of Theorem 10.10 would become a bit easier if one replaced $\operatorname{Spin}(2) \times \operatorname{Spin}(2)$ by $\operatorname{Spin}(2) . \operatorname{Spin}(2)$ in Definition 10.1. The setup we chose, on the other hand, makes it easier to deal with reducible diagrams. Of course, one could a priori try to just restrict oneself to the case of irreducible diagrams, which in the case of simply laced diagrams is unproblematic. However, when dealing with arbitrary diagams it will turn out that it is more natural to also allow reducible diagrams.

\section{SPIN COVERS OF SIMPLY LACED TYPE}

Notation 11.1. Let $\Pi=(V, E)$ be a (finite) simply laced diagram with labelling $\sigma: I \rightarrow V$ and let $c(\Pi)$ denote the number of connected components of $\Pi$. A component labelling of $\Pi$ is a map $\mathcal{K}: V \rightarrow\{1, \ldots, c(\Pi)\}$ such that $u, v \in V$ are in the same connected component of $\Pi$ if and only if $\mathcal{K}(u)=\mathcal{K}(v)$.

Throughout this section, let $\Pi$ be a (finite) simply laced diagram, $\sigma: I \rightarrow V$ and labelling and $\mathcal{K}$ a component labelling.

Generalizing Theorem 9.9 the universal enveloping group of a continuous $\mathrm{SO}(2)$-amalgam over an arbitrary simply laced diagram $\Pi$ is isomorphic to the maximal compact subgroup of the corresponding split real Kac-Moody group (cf. Theorem 11.2). The goal of this section is to construct and investigate its spin cover, which will arise as the universal enveloping group of the continuous Spin(2)-amalgam over the same simply laced diagram $\Pi$. In the case of $E_{10}$ its existence has been conjectured by Damour and Hillmann in DH09, Section 3.5, p. 24].

Additional key ingredients, next to transitive actions on buildings and the theory of $\mathrm{SO}(2)-$ and Spin(2)-amalgams developed so far, will be the generalized spin representations constructed in [HKL13] and the Iwasawa decomposition of split real Kac-Moody groups studied, for example, in [DMGH09]. For definitions and details on Kac-Moody theory we refer the reader to [Kac90], [Rém02], HKL13, Section 2], [HKM13, Section 7], Mar13, Chapter 5].

Theorem 11.2. Let $\Pi$ be a simply laced diagram, let $G(\Pi)$ be the corresponding simply connected split real Kac-Moody group, and let $K(\Pi)$ be its maximal compact subgroup, i.e., the subgroup fixed by the CartanChevalley involution. Then there exists a faithful universal enveloping morphism $\tau_{K(\Pi)}: \mathcal{A}(\Pi, \mathrm{SO}(2)) \rightarrow$ $K(\Pi)$.

Proof. For $i \in I$ denote by $G_{i}$ the fundamental rank one subgroups of $G(\Pi)$ and, for $i \neq j \in I$, by $G_{i j}$ the fundamental rank two subgroups. The groups $G_{i}$ are isomorphic to $\mathrm{SL}_{2}(\mathbb{R})$ and the groups $G_{i j}$ are isomorphic to $\mathrm{SL}_{3}(\mathbb{R})$ or to $\mathrm{SL}_{2}(\mathbb{R}) \times \mathrm{SL}_{2}(\mathbb{R})$, depending on whether the vertices $i^{\sigma}, j^{\sigma}$ of $\Pi$ are joined by an edge or not. The Cartan-Chevalley involution $\omega$ leaves the groups $G_{i}, G_{i j}$ setwise invariant and, in fact, induces the transpose-inverse map on these groups. Define $H_{i}:=\operatorname{Fix}_{G_{i}}(\omega) \cong \operatorname{SO}(2)$ and $H_{i j}:=\operatorname{Fix}_{G_{i j}}(\omega)$, the latter being isomorphic to $\mathrm{SO}(3)$ or to $\mathrm{SO}(2) \times \mathrm{SO}(2)$, and let $\psi_{i j}^{i}: H_{i} \rightarrow H_{i j}$ denote the (continuous) inclusion map for $i \neq j \in I$. By DMGH09, Theorem 1.2], the group $K(\Pi)$ is the universal enveloping group of the amalgam $\mathcal{A}_{1}:=\left\{H_{i j}, \psi_{i j}^{i}, \mid i \neq j \in I\right\}$.

Given $i<j \in I$ such that $\{i, j\}^{\sigma} \in E(\Pi)$, by Theorem 9.8 applied to the subdiagram of $\Pi$ of type $A_{2}$ consisting of the vertices $i^{\sigma}, j^{\sigma}$ there is a continuous isomorphism $\alpha_{i j}: H_{i j} \rightarrow \mathrm{SO}(3)$ such that

$$
\left(\alpha_{i j} \circ \psi_{i j}^{i}\right)\left(H_{i}\right)=\varepsilon_{12}(\mathrm{SO}(2)), \quad\left(\alpha_{i j} \circ \psi_{i j}^{j}\right)\left(H_{j}\right)=\varepsilon_{23}(\mathrm{SO}(2)) \ldots
$$

Let $i \in I$ and choose $j \in I$ such that $\{i, j\}^{\sigma} \in E(\Pi)$. Define

$$
\alpha_{i}:= \begin{cases}\varepsilon_{12}^{-1} \circ \alpha_{i j} \circ \psi_{i j}^{i}: H_{i} \rightarrow \mathrm{SO}(2), & \text { if } i>j, \\ \varepsilon_{23}^{-1} \circ \alpha_{i j} \circ \psi_{i j}^{i}: H_{i} \rightarrow \mathrm{SO}(2), & \text { if } i<j .\end{cases}
$$

For $i<j \in I$ such that $\{i, j\}^{\sigma} \notin E(\Pi)$, define $\alpha_{i j}:=\alpha_{i} \times \alpha_{j}: H_{i j}=H_{i} \times H_{j} \rightarrow \operatorname{SO}(2) \times \operatorname{SO}(2)$. For arbitrary $i \neq j \in I$, let

and

$$
K_{i j}:= \begin{cases}\mathrm{SO}(3), & \text { if }\{i, j\}^{\sigma} \in E(\Pi), \\ \mathrm{SO}(2) \times \mathrm{SO}(2), & \text { if }\{i, j\}^{\sigma} \notin E(\Pi),\end{cases}
$$

$$
\bar{\phi}_{i j}^{i}:=\alpha_{i j} \circ \psi_{i j}^{i} \circ \alpha_{i}^{-1}: \mathrm{SO}(2) \rightarrow K_{i j}
$$


Then $\mathcal{A}_{2}:=\left\{K_{i j}, \bar{\phi}_{i j}^{i}, \mid i \neq j \in I\right\}$ is an $\mathrm{SO}(2)$-amalgam with respect to $\Pi$ and $\sigma$. Moreover, the system $\alpha=\left\{\operatorname{id}_{I}, \alpha_{i j}, \alpha_{i} \mid i \neq j \in I\right\}: \mathcal{A}_{1} \rightarrow \mathcal{A}_{2}$ is an isomorphism of amalgams. Indeed, given $i \neq j \in I$, one has $\bar{\phi}_{i j}^{i} \circ \alpha_{i}=\alpha_{i j} \circ \psi_{i j}^{i} \circ \alpha_{i}^{-1} \circ \alpha_{i}=\alpha_{i j} \circ \psi_{i j}^{i}$. Finally, $\alpha_{i}$ is continuous for each $i \in I$, whence $\bar{\phi}_{i j}^{i}$ is continuous for all $i \neq j \in I$. Therefore, $\mathcal{A}_{2}$ is a continuous $\mathrm{SO}(2)$-amalgam with respect to $\Pi$ and $\sigma$ so that $\mathcal{A}_{1} \cong \mathcal{A}_{2} \cong \mathcal{A}(\Pi, \mathrm{SO}(2))$ by Theorem 9.8

Notation 11.3. For consistency, we fix the groups and connecting morphisms in the standard $\mathrm{SO}(2)$ amalgam with respect to $\Pi$ as follows (cf. Definition 9.6 ):

$$
\mathcal{A}(\Pi, \mathrm{SO}(2))=\left\{K_{i j}, \phi_{i j}^{i}, \mid i \neq j \in I\right\} .
$$

Similarly for the standard Spin(2)-amalgam with respect to $\Pi$ (cf. Definition 10.8):

$$
\mathcal{A}(\Pi, \operatorname{Spin}(2))=\left\{\widetilde{K}_{i j}, \tilde{\phi}_{i j}^{i} \mid i \neq j \in I\right\} .
$$

We denote the epimorphism of amalgams from Remark 10.5 by

$$
\pi_{\Pi}: \mathcal{A}(\Pi, \operatorname{Spin}(2)) \rightarrow \mathcal{A}(\Pi, \mathrm{SO}(2)) .
$$

Remark 11.4. As discussed in Remark 9.2 the amalgam $\mathcal{A}(\Pi, \mathrm{SO}(2))$ consists of compact Lie groups with continuous connecting homomorphisms. On the other hand, the group $K(\Pi)$ naturally carries a Hausdorff group topology that is $k_{\omega}$ : Indeed, $K(\Pi)$ is the subgroup of the unitary form studied in [GGH10, Section 6] fixed by complex conjugation and it is the subgroup of the real Kac-Moody group $G(\Pi)$ studied in [HKM13, Section 7] fixed by the Cartan-Chevalley involution; both ambient groups are $k_{\omega}$ (by [GGH10, Theorem 6.12], resp. HKM13, Theorem 7.22]) and, hence, so is any subgroup fixed by a continuous involution (cf. GGH10, Proposition 4.2(b)]). Note that the $k_{\omega}$-group topologies on $K(\Pi)$ induced from the real Kac-Moody group $G(\Pi)$ and from the unitary form coincide, as both are induced from the $k_{\omega}$-group topology on the ambient complex Kac-Moody group (cf. [GGH10, Theorem 6.3], resp. [HKM13, Theorem 7.22]).

Furthermore, a straightforward adaption of the proof of [GGH10, Proposition 6.9] implies that this $k_{\omega}$-group topology is the finest group topology with respect to the enveloping homomorphisms $\tau_{i j}: K_{i j} \rightarrow$ $K(\Pi)$. In other words, the obvious analog of [GGH10, Theorem 6.12], [HKM13, Theorem 7.22] holds for $\left(K(\Pi), \tau_{\Pi}\right)$. In particular, to any enveloping morphism $\phi=\left(\phi_{i j}\right): \mathcal{A}(\Pi, \mathrm{SO}(2)) \rightarrow H$ into a Hausdorff topological group $H$ with continuous homomorphisms $\phi_{i j}: K_{i j} \rightarrow H$ there exists a unique continuous homomorphism $\phi: K(\Pi) \rightarrow H$ such that the following diagram commutes:

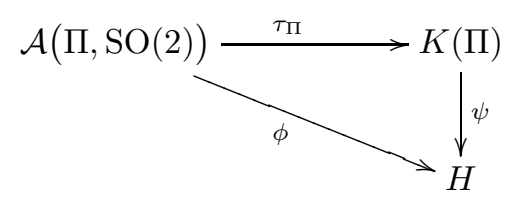

Theorems 9.9 and 10.10 state that the double cover $\operatorname{Spin}(n)$ of $\mathrm{SO}(n)$ is the universal enveloping group of the two-fold central extension $\mathcal{A}\left(A_{n-1}, \operatorname{Spin}(2)\right)$ of the amalgam $\mathcal{A}\left(A_{n-1}, \mathrm{SO}(2)\right)$ as defined in Proposition 3.9. In view of Theorem 11.2 it is therefore natural to introduce the following notion.

Definition 11.5. The spin group $\operatorname{Spin}(\Pi)$ with respect to $\Pi$ is the canonical universal enveloping group of the (continuous) amalgam $\mathcal{A}(\Pi, \operatorname{Spin}(2))$ with the canonical universal enveloping morphism

$$
\tau_{\operatorname{Spin}(\Pi)}=\left\{\tau_{i j} \mid i \neq j \in I\right\}: \mathcal{A}(\Pi, \operatorname{Spin}(2)) \rightarrow \operatorname{Spin}(\Pi) .
$$

Lemma 11.6. $K(\Pi)$ is an enveloping group of the amalgam $\mathcal{A}(\Pi, \operatorname{Spin}(2))$. There exists a unique central extension $\rho_{\Pi}: \operatorname{Spin}(\Pi) \rightarrow K(\Pi)$ that makes the following diagram commute (cf. Notation 3.8):

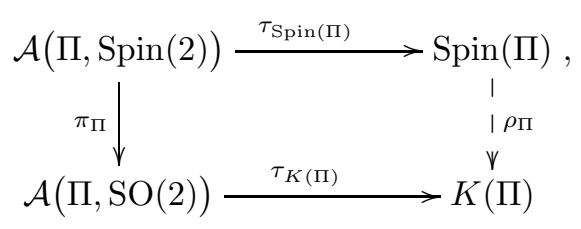

where

$$
\tau_{K(\Pi)}=\left\{\psi_{i j}: K_{i j} \rightarrow K(\Pi) \mid i \neq j \in I\right\}: \mathcal{A}(\Pi, \mathrm{SO}(2)) \rightarrow K(\Pi)
$$

is the universal enveloping morphism whose existence is guaranteed by Theorem 11.2. 
Proof. As in Definition 10.3, for $i \neq j \in I$ let $\rho_{i j}: \widetilde{K}_{i j} \rightarrow K_{i j}$ be the epimorphism $\rho_{3}$ if $\{i, j\}^{\sigma}$ is an edge, and $\rho_{2} \times \rho_{2}$ otherwise. Then, by Lemma $3.7(\mathrm{a})$, the group $K(\Pi)$ with the homomorphisms $\xi_{i j}:=\psi_{i j} \circ \rho_{i j}: \widetilde{K}_{i j} \rightarrow K(\Pi)$ for all $i \neq j \in I$ is an enveloping group of $\mathcal{A}(\Pi, \sigma, \operatorname{Spin}(2))$. By universality of $\tau_{\operatorname{Spin}(\Pi)}: \mathcal{A}(\Pi, \operatorname{Spin}(2)) \rightarrow \operatorname{Spin}(\Pi)$, Lemma $3.7(\mathrm{~b})$ provides a unique epimorphism $\rho_{\Pi}: \operatorname{Spin}(\Pi) \rightarrow K(\Pi)$ with the properties as claimed. This epimorphism is a central extension by Proposition 3.9.

The following is a straightforward generalization of the observation we made towards the end of the proof of Theorem 10.10 .

Lemma 11.7. Let $i \neq j \in I$ and $k \neq \ell \in I$. If $i^{\sigma}$ and $k^{\sigma}$ are in the same connected component of $\Pi$, then

$$
\tau_{i j}\left(\tilde{\phi}_{i j}^{i}\left(-1_{\operatorname{Spin}(2)}\right)\right)=\tau_{k \ell}\left(\tilde{\phi}_{k \ell}^{k}\left(-1_{\operatorname{Spin}(2)}\right)\right) .
$$

Proof. As $i^{\sigma}$ and $k^{\sigma}$ are in the same connected component, there exists a sequence $i_{0}:=i, i_{1}, \ldots, i_{n}:=$ $k \in I$ such that $\left\{i_{r}^{\sigma}, i_{r+1}^{\sigma}\right\}$ are edges for $0 \leq r<n$. Thus $\widetilde{K}_{i_{r} i_{r+1}}=\operatorname{Spin}(3)$ and by the definition of $\operatorname{Spin}(\Pi)$ as the canonical universal enveloping group of the amalgam $\mathcal{A}(\Pi, \operatorname{Spin}(2))$, we have

Hence

$$
\left.\left.\tilde{\phi}_{i_{r} i_{r+1}}^{i_{r}}\left(-1_{\operatorname{Spin}(2)}\right)\right)=-1_{\operatorname{Spin}(3)}=\tilde{\phi}_{i_{r} i_{r+1}}^{i_{r+1}}\left(-1_{\operatorname{Spin}(2)}\right)\right) \text {. }
$$

$$
\begin{aligned}
\tau_{i j}\left(\tilde{\phi}_{i j}^{i}\left(-1_{\operatorname{Spin}(2)}\right)\right) & =\tau_{i_{0} i_{1}}\left(\tilde{\phi}_{i_{0} i_{1}}^{i_{0}}\left(-1_{\operatorname{Spin}(2)}\right)\right)=\tau_{i_{0} i_{1}}\left(\tilde{\phi}_{i_{0} i_{1}}^{i_{1}}\left(-1_{\operatorname{Spin}(2)}\right)\right)=\cdots \\
& =\tau_{i_{n-1} i_{n}}\left(\tilde{\phi}_{i_{n-1} i_{n}}\left(-1_{\operatorname{Spin}(2)}\right)\right)=\tau_{i_{n-1} k}\left(\tilde{\phi}_{i_{n-1} k}^{k}\left(-1_{\operatorname{Spin}(2)}\right)\right) \\
& =\tau_{k \ell}\left(\tilde{\phi}_{k \ell}^{k}\left(-1_{\operatorname{Spin}(2)}\right)\right) .
\end{aligned}
$$

where the first and last equality hold due to the definition of enveloping homomorphisms.

Thus the following is well-defined.

Definition 11.8. For $i \neq j \in I$ define

$$
-1_{\operatorname{Spin}(\Pi), \mathcal{K}(i)}:=\tau_{i j}\left(\tilde{\phi}_{i j}^{i}\left(-1_{\operatorname{Spin}(2)}\right)\right) \quad \text { and } \quad Z:=\left\langle-1_{\operatorname{Spin}(\Pi), 1}, \ldots,-1_{\operatorname{Spin}(\Pi), c(\Pi)}\right\rangle \leq \operatorname{Spin}(\Pi) .
$$

Observation 11.9. The following are true:

(a) $Z$ is contained in the centre of $\operatorname{Spin}(\Pi)$.

(b) $|Z| \leq 2^{c(\Pi)}$.

The first assertion is immediate from Proposition 3.9 applied to the $\operatorname{Spin}(2)$-amalgam $\mathcal{A}(\Pi, \operatorname{Spin}(2))$ and the $\mathrm{SO}(2)$-amalgam $\mathcal{A}(\Pi, \mathrm{SO}(2))$ with $\widetilde{U}=\operatorname{Spin}(2), \widetilde{V}=\langle-1\rangle$ and $U=\mathrm{SO}(2)$. The second follows from the fact that $Z$ is abelian by assertion (a) and admits a generating system of $c(\Pi)$ involutions by definition.

The remainder of this section is mostly devoted to proving the following result:

Theorem 11.10. One has $|Z|=2^{c(\Pi)}$.

We start the proof of this theorem by revisiting Remark 6.5.

Lemma 11.11. Let $V$ be an $\mathbb{R}$-vector space and let $X_{i}, X_{j} \in \operatorname{End}(V)$ be such that

$$
X_{i}^{2}=-\mathrm{id}_{V}=X_{j}^{2}, \quad X_{i} X_{j}=-X_{j} X_{i} .
$$

Then the map

$$
\begin{aligned}
\psi: \operatorname{Spin}(3) & \rightarrow \mathrm{GL}(V) \\
a+b e_{1} e_{2}+c e_{2} e_{3}+d e_{1} e_{3} & \mapsto a \operatorname{id}_{V}+b X_{i}+c X_{j}+d X_{i} X_{j}
\end{aligned}
$$

is a group monomorphism such that

$$
\psi\left(\tilde{\varepsilon}_{12}(S(\alpha))\right)=\cos (\alpha) \operatorname{id}_{V}+\sin (\alpha) X_{i}, \quad \psi\left(\tilde{\varepsilon}_{23}(S(\alpha))\right)=\cos (\alpha) \operatorname{id}_{V}+\sin (\alpha) X_{j} .
$$

Proof. The subspace $\mathbb{H}:=\left\langle\operatorname{id}_{V}, X_{i}, X_{j}, X_{i} X_{j}\right\rangle_{\mathbb{R}}$ is an $\mathbb{R}$-subalgebra of $\operatorname{End}(V)$, the set $\left\{\operatorname{id}_{V}, X_{i}, X_{j}, X_{i} X_{j}\right\}$ is an $\mathbb{R}$-basis of $\mathbb{H}$, and the $\mathbb{R}$-linear extension $\hat{\psi}: \mathrm{Cl}(3)^{0} \rightarrow \mathbb{H}$ of

$$
1 \mapsto \operatorname{id}_{V}, \quad e_{1} e_{2} \mapsto X_{i}, \quad e_{1} e_{3} \mapsto X_{i} X_{j}, \quad e_{2} e_{3} \mapsto X_{j}
$$

is an isomorphism of algebras: Indeed, since $\mathrm{id}_{V}, X_{i}, X_{j}$ and $X_{i} X_{j}$ satisfy the same relations as $1, e_{1} e_{2}$, $e_{2} e_{3}$ and $e_{1} e_{3}$, the map $\hat{\psi}$ is a homomorphism of rings. Since $X_{i} \neq 0_{\operatorname{End}(V)}$, one has $\operatorname{ker}(\hat{\psi}) \neq \operatorname{Cl}(3)^{0}$. By Remark 6.5 $\mathrm{Cl}(3)^{0}$ is a skew field and, thus, simple as a ring. Therefore, $\hat{\psi}$ is injective and, hence, bijective, because $\operatorname{dim}_{\mathbb{R}} \mathbb{H} \leq 4$, i.e., $\hat{\psi}$ is an isomorphism of algebras. 
Consequently, the restriction $\psi$ of $\hat{\psi}$ to $\operatorname{Spin}(3)$ is injective with values in $\operatorname{GL}(V)$, i.e., $\psi: \operatorname{Spin}(3) \rightarrow$ $\mathrm{GL}(V)$ is a group monomorphism. The final statement is immediate from the definitions.

Lemma 11.12. Let $V$ be an $\mathbb{R}$-vector space and let $X_{i}, X_{j} \in \operatorname{End}(V)$ be such that

$$
X_{j} \notin\left\langle\operatorname{id}_{V}, X_{i}\right\rangle_{\mathbb{R}}, \quad X_{i}^{2}=-\mathrm{id}_{V}=X_{j}^{2}, \quad X_{i} X_{j}=X_{j} X_{i} .
$$

Then the map

$$
\begin{aligned}
\psi:\left\langle\tilde{\varepsilon}_{12}(\operatorname{Spin}(2)), \tilde{\varepsilon}_{34}(\operatorname{Spin}(2))\right\rangle \subseteq \operatorname{Spin}(4) & \rightarrow \mathrm{GL}(V) \\
a+b e_{1} e_{2}+c e_{3} e_{4}+d e_{1} e_{2} e_{3} e_{4} & \mapsto a \operatorname{id}_{V}+b X_{i}+c X_{j}+d X_{i} X_{j}
\end{aligned}
$$

is a group monomorphism such that

$$
\psi\left(\tilde{\varepsilon}_{12}(S(\alpha))\right)=\cos (\alpha) \operatorname{id}_{V}+\sin (\alpha) X_{i}, \quad \psi\left(\tilde{\varepsilon}_{34}(S(\alpha))\right)=\cos (\alpha) \operatorname{id}_{V}+\sin (\alpha) X_{j} .
$$

Proof. The subspace $\mathbb{A}:=\left\langle\operatorname{id}_{V}, X_{i}, X_{j}, X_{i} X_{j}\right\rangle_{\mathbb{R}}$ is an $\mathbb{R}$-subalgebra of $\operatorname{End}(V)$, the set $\left\{\operatorname{id}_{V}, X_{i}, X_{j}, X_{i} X_{j}\right\}$ is an $\mathbb{R}$-basis of $\mathbb{A}$, and the $\mathbb{R}$-linear extension $\hat{\psi}: \tilde{\mathbb{A}}:=\left\langle 1, e_{1} e_{2}, e_{3} e_{4}, e_{1} e_{2} e_{3} e_{4}\right\rangle_{\mathbb{R}} \subseteq \mathrm{Cl}(4)^{0} \rightarrow \mathbb{A}$ of

$$
1 \mapsto \operatorname{id}_{V}, \quad e_{1} e_{2} \mapsto X_{i}, \quad e_{3} e_{4} \mapsto X_{j}, \quad e_{1} e_{2} e_{3} e_{4} \mapsto X_{i} X_{j}
$$

is an isomorphism of algebras: Indeed, since $\mathrm{id}_{V}, X_{i}, X_{j}$ and $X_{i} X_{j}$ satisfy the same relations as $1, e_{1} e_{2}$, $e_{3} e_{4}$ and $e_{1} e_{2} e_{3} e_{4}$, the map $\psi$ is a homomorphism of rings. The hypothesis $X_{j} \notin\left\langle\operatorname{id}_{V}, X_{i}\right\rangle_{\mathbb{R}}$ implies that

$$
\left\{1, X_{i}, X_{j}, X_{i} X_{j}\right\}
$$

is $\mathbb{R}$-linearly independent. Therefore, $\hat{\psi}$ is injective and, thus, bijective, because $\operatorname{dim}_{\mathbb{R}} \mathbb{A} \leq 4$.

Consequently, the restriction $\psi$ of $\hat{\psi}$ to $\left\langle\tilde{\varepsilon}_{12}(\operatorname{Spin}(2)), \tilde{\varepsilon}_{34}(\operatorname{Spin}(2))\right\rangle \subseteq \operatorname{Spin}(4)$ is injective with values in $\operatorname{GL}(V)$, i.e., $\psi:\left\langle\tilde{\varepsilon}_{12}(\operatorname{Spin}(2)), \tilde{\varepsilon}_{34}(\operatorname{Spin}(2))\right\rangle \subseteq \operatorname{Spin}(4) \rightarrow \operatorname{GL}(V)$ is a group monomorphism as claimed. The final statement is immediate from the definitions.

Remark 11.13. We are now in a position to use the results of [HKL13] in order to confirm the conjecture concerning $\operatorname{Spin}(\Pi)$ made in footnote 18 on page 24 of [DH09]. The definition of a generalized spin representation can be found in [HKL13, Definition 4.4], the definition and existence of a maximal one in HKL13, Corollary 4.8].

We point out that HKL13, Example 4.1] uses a convention for Clifford algebras different from the one used in the present article; however, HKL13, Corollary 4.8] is formulated and proved without making any reference to Clifford algebras whatsoever.

Theorem 11.14. Let

- $\Pi$ be an irreducible simply laced diagram with labelling $\sigma: I \rightarrow V$,

- $\mathfrak{g}$ be the Kac-Moody algebra corresponding to $\Pi$ and $\mathfrak{k}$ its maximal compact subalgebra with Berman generators $Y_{1}, \ldots, Y_{n}$ (cf. HKL13, Section 2.2]),

- $\mu: \mathfrak{k} \rightarrow \operatorname{End}\left(\mathbb{C}^{s}\right), s \in \mathbb{N}$, be a maximal generalized spin representation (cf. HKL13, Corollary 4.8]), and

- $X_{i}:=2 \mu\left(Y_{i}\right)$ for each $i \in I$.

Then, for each $i \neq j \in I$, there exist subgroups $H_{i j} \leq \mathrm{GL}_{\mathrm{S}}(\mathbb{C})$ and an enveloping morphism

$$
\Psi_{\mathcal{A}}=\left\{\psi_{i j} \mid i \neq j \in I\right\}: \mathcal{A}(\Pi, \operatorname{Spin}(2)) \rightarrow H:=\left\langle H_{i j} \mid i \neq j \in I\right\rangle
$$

with injective $\psi_{i j}$ whenever $\{i, j\}^{\sigma} \in E(\Pi)$.

Proof. According to [HKL13, Remark 4.5], given $i \neq j \in I$, one has $X_{i}^{2}=-\mathrm{id}_{V}=X_{j}^{2}$, and

$$
X_{i} X_{j}= \begin{cases}-X_{j} X_{i}, & \text { if }\{i, j\}^{\sigma} \in E(\Pi), \\ X_{j} X_{i}, & \text { if }\{i, j\}^{\sigma} \notin E(\Pi) .\end{cases}
$$

Moreover, $X_{j} \notin\left\langle\operatorname{id}_{V}, X_{i}\right\rangle_{\mathbb{R}}$, as $\mu$ is maximal. Thus Lemma 11.11 provides group monomorphisms $\psi_{i j}$ : $\widetilde{K}_{i j} \rightarrow \mathrm{GL}_{s}(\mathbb{C})$, if $\{i, j\}^{\sigma} \in E(\Pi)$, and Lemma 11.12 provides group homomorphisms $\psi_{i j}: \widetilde{K}_{i j} \rightarrow \mathrm{GL}_{s}(\mathbb{C})$ with kernel $\langle-1,-1\rangle$, if $\{i, j\}^{\sigma} \notin E(\Pi)$. This allows one to define

$$
H_{i j}:=\operatorname{im}\left(\psi_{i j}\right) .
$$

Restriction of the ranges of the maps $\psi_{i j}$ to $H_{i j}$ thus provides

$$
\begin{array}{lll}
\text { group isomorphisms } & \psi_{i j}: \widetilde{K}_{i j}=\operatorname{Spin}(3) \rightarrow H_{i j}, & \text { if }\{i, j\}^{\sigma} \in E(\Pi), \\
\text { group epimorphisms } & \psi_{i j}: \widetilde{K}_{i j}=\operatorname{Spin}(2) \times \operatorname{Spin}(2) \rightarrow H_{i j}, & \text { if }\{i, j\}^{\sigma} \notin E(\Pi),
\end{array}
$$


satisfying

$$
\forall i \neq j \in I: \quad \psi_{i j}\left(\tilde{\phi}_{i j}^{j}\left(\cos (\alpha)+\sin (\alpha) e_{1} e_{2}\right)\right)=\cos (\alpha) \mathrm{id}_{V}+\sin (\alpha) X_{j} .
$$

In particular, one has

$$
\forall i \neq j \neq k: \quad \psi_{i j} \circ \tilde{\phi}_{i j}^{j}=\psi_{k j} \circ \tilde{\phi}_{k j}^{j} .
$$

The set $\Psi_{\mathcal{A}}:=\left\{\psi_{i j} \mid i \neq j \in I\right\}$ is the desired enveloping morphism.

Remark 11.15. Let everything be as in Theorem 11.14, By universality of $\tau_{\operatorname{Spin}(\Pi)}: \mathcal{A}(\Pi, \operatorname{Spin}(2)) \rightarrow$ Spin(I) (cf. Definition 11.5) there exists an epimorphism

$$
\Xi: \operatorname{Spin}(\Pi) \rightarrow H
$$

such that the following diagram commutes:

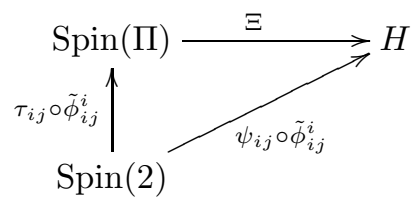

The commutative diagram in Lemma 11.6 and the finiteness of the central extension $\operatorname{Spin}(\Pi) \rightarrow K(\Pi)$ by Observation 11.9 in fact allow one to lift the topological universality statement from Remark 11.4 concerning $\tau_{\Pi}: \mathcal{A}(\Pi, \mathrm{SO}(2)) \rightarrow K(\Pi)$ to a topological universality statement concerning $\tau_{\operatorname{Spin}(\Pi)}$ : $\mathcal{A}(\Pi, \operatorname{Spin}(2)) \rightarrow \operatorname{Spin}(\Pi)$. Moreover, the maps $\psi$ constructed in Lemmas 11.11 and 11.12 are certainly continuous with respect to the Lie group topologies, if $\operatorname{dim}_{\mathbb{R}}(V)<\infty$.

In particular, the enveloping morphism $\Psi_{\mathcal{A}}=\left\{\psi_{i j}\right\}$ from the theorem consists of continuous maps, so that by universality $\Xi: \operatorname{Spin}(\Pi) \rightarrow H$ is continuous as well.

As an immediate consequence we record:

Corollary 11.16. Let $\Pi$ be an irreducible simply laced diagram. Then $1_{\operatorname{Spin}(\Pi)} \neq-1_{\operatorname{Spin}(\Pi)}$.

Proof. We conclude from Remark 11.15

$$
\Xi\left(-1_{\operatorname{Spin}(\Pi)}\right)=\left(\Xi \circ \tau_{12} \circ \tilde{\phi}_{12}^{1}\right)\left(-1_{\operatorname{Spin}(2)}\right)=\left(\xi_{12} \circ \tilde{\phi}_{12}^{1}\right)\left(-1_{\operatorname{Spin}(2)}\right)=\cos (\pi) \mathrm{id}_{V}+\sin (\pi) X_{1}=-\mathrm{id}_{V},
$$

and, hence, $1_{\operatorname{Spin}(\Pi)} \neq-1_{\operatorname{Spin}(\Pi)}$.

Theorem 11.17. Let $\Pi$ be a simply laced diagram. Then the universal enveloping group

$$
\left(\operatorname{Spin}(\Pi), \tau_{\operatorname{Spin}(\Pi)}=\left\{\tau_{i j} \mid i \neq j \in I\right\}\right)
$$

of $\mathcal{A}(\Pi, \operatorname{Spin}(2))$ is a $2^{c(\Pi)}$-fold central extension of the universal enveloping group $K(\Pi)$ of $\mathcal{A}(\Pi, \mathrm{SO}(2))$.

Proof. Let $\Pi_{1}, \ldots, \Pi_{c(\Pi)}$ be the connected components of $\Pi$. Then

$$
\operatorname{Spin}(\Pi)=\operatorname{Spin}\left(\Pi_{1}\right) \times \cdots \times \operatorname{Spin}\left(\Pi_{c(\Pi)}\right)
$$

Indeed,

$$
\begin{array}{rlrl}
\tau_{\operatorname{Spin}(\Pi)}: \mathcal{A}(\Pi, \operatorname{Spin}(2)) & \rightarrow \operatorname{Spin}\left(\Pi_{1}\right) \times \cdots \times \operatorname{Spin}\left(\Pi_{c(\Pi)}\right) & \\
\tau_{i j}: \widetilde{K}_{i j} \rightarrow \tau_{i j}\left(\widetilde{K}_{i j}\right) & & \text { if } \mathcal{K}(i)=\mathcal{K}(j) \\
\tau_{i j}: \widetilde{K}_{i j} \rightarrow\left(\tau_{i j} \circ \tilde{\phi}_{i j}^{i}\right)(\operatorname{Spin}(2)) \times\left(\tau_{i j} \circ \tilde{\phi}_{i j}^{j}\right)(\operatorname{Spin}(2)):(x, y) \mapsto \tau_{i j}(x, y) & \text { if } \mathcal{K}(i) \neq \mathcal{K}(j)
\end{array}
$$

is an enveloping morphism.

It therefore suffices to prove the theorem for irreducible simply laced diagrams $\Pi$. In this case, however, it is immediate from Proposition 3.9 applied to the $\widetilde{U}$-amalgam $\mathcal{A}(\Pi, \operatorname{Spin}(2))$ and the $U$-amalgam $\mathcal{A}(\Pi, \mathrm{SO}(2))$ with $\widetilde{U}=\operatorname{Spin}(2), U=\mathrm{SO}(2)$ and $\widetilde{V}=\langle-1\rangle$ combined with Lemma 11.7 and Corollary 11.16.

We have proved Theorem 11.10 and Theorem $\mathrm{A}$ from the introduction. 


\section{Part III. Non-simply laced rank two diagrams}

\section{Strategies for Reducing the General CASE to the Simply-LaCEd one}

Until now we exclusively studied spin covers of maximal compact subgroups of split real Kac-Moody groups of simply laced type. Our next goal is to generalize this concept to arbitrary Dynkin diagrams resp. generalized Cartan matrices. We pursue this goal via two strategies: The first one is via epimorphisms between maximal compact subgroups induced by local epimorphisms on amalgam-level in rank two where we replace non-simple edges by non-edges, simple edges or double edges; the second one is via embeddings into larger groups by unfolding the diagrams resp. the Cartan matrices to simply-laced cover diagrams as in HKL13.

The first strategy will allow us to transform arbitrary Dynkin diagrams resp. generalized Cartan matrices into doubly laced ones. The second strategy will work for the resulting doubly laced generalized Cartan matrices. A combination of both strategies allows us to deal with arbitrary generalized Cartan matrices.

Strategy 12.1. In order to deal with the two non-simply laced spherical diagrams of rank two $-\mathrm{C}_{2}$ and $\mathrm{G}_{2}$ - we consider point-line models of the Tits buildings of the split real Lie groups $\mathrm{Sp}_{4}(\mathbb{R})$ and $\mathrm{G}_{2}(2)$, the so-called symplectic quadrangle and the so-called split Cayley hexagon. As in the proof of Theorem 9.9 the Iwasawa decomposition implies that the maximal compact subgroups $\mathrm{U}_{2}(\mathbb{C}) \leq \mathrm{Sp}_{4}(\mathbb{R})$ and $\mathrm{SO}(4) \leq \mathrm{G}_{2}(2)$ act flag-transitively on the respective point-line geometries.

Their unique double covers $\mathrm{SO}(2) \times \mathrm{SU}_{2}(\mathbb{C}) \rightarrow \mathrm{U}_{2}(\mathbb{C})$ and $\mathrm{Spin}(4) \rightarrow \mathrm{SO}(4)$ fit into the commutative diagrams

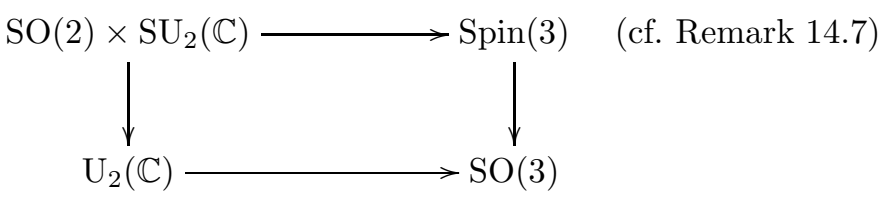

and

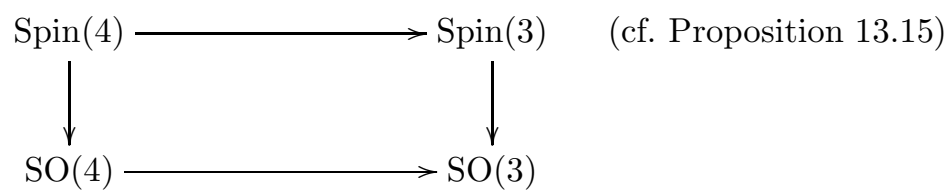

which allow one to transform point and line stabilizers in $\mathrm{U}_{2}(\mathbb{C})$ and $\mathrm{SO}(4)$ into point, resp. line stabilizers in $\mathrm{SO}(3)$ in a way that is compatible with the covering maps. This in turn will allow us to transform a Spin(2)-amalgam for a given two-spherical diagram $\Pi$ into a $\operatorname{Spin}(2)$-amalgam for the simply laced diagram $\Pi^{\mathrm{sl}}$ that one obtains from $\Pi$ by replacing all edges by simple edges. As a consequence - based on Theorem 11.17 — in Theorem 17.1 below we will be able to prove that the spin cover $\operatorname{Spin}(\Pi)$ is a non-trivial central extension of $K(\Pi)$ for suitable two-spherical diagrams $\Pi$.

As a caveat we point out that the compatibility of the covering maps in the $\mathrm{C}_{2}$ case is quite subtle and actually fails under certain circumstances, due to the phenomena described in Lemma 14.10. In order to control these subtleties we introduce the notion of admissible colourings of Dynkin diagrams in Definition 16.2. These subtleties are also why we actually only replace certain double edges by single edges and additionally employ Strategy 12.2 below.

When trying to deal with non-two-spherical diagrams further subtleties arise. The non-spherical Cartan matrices of rank two are of the form

$$
\left(\begin{array}{cc}
2 & -r \\
-s & 2
\end{array}\right)
$$

for $r, s \in \mathbb{N}$ such that $r s \geq 4$. The isomorphism type of the maximal compact subgroup $K$ of the corresponding split real Kac-Moody group depends (only) on the parities of $r$ and $s$. Indeed, in all cases $K$ is isomorphic to a free amalgamated product

$$
K \cong K_{1} T_{K} *_{T_{K}} K_{2} T_{K}
$$

where $K_{1} \cong \mathrm{SO}(2) \cong K_{2}$ with $T_{K}=\left\{1, t_{1}, t_{2}, t_{1} t_{2}\right\} \cong \mathbb{Z} / 2 \mathbb{Z} \times \mathbb{Z} / 2 \mathbb{Z}$ and $T_{K} \cap K_{1}=\left\langle t_{1}\right\rangle, T_{K} \cap K_{2}=\left\langle t_{2}\right\rangle$ and $K_{i} \unlhd K_{i} T_{K}$. We conclude that the isomorphism type of $K$ is known once the action of $t_{1}$ on $K_{2}$ and the action of $t_{2}$ on $K_{1}$ are known. It turns out that $t_{1}$ centralizes $K_{2}$ if and only if $r$ is even and inverts $K_{2}$ if and only if $r$ is odd; similarly, $t_{2}$ centralizes $K_{1}$ if and only if $s$ is even and inverts $K_{1}$ if and only if $s$ is odd (cf. Remark 15.4). 
To these four cases of parities of $r$ and $s$ correspond three cases of epimorphisms from $K$ onto compact Lie groups: $K \rightarrow \mathrm{SO}(2) \times \mathrm{SO}(2)$, if both $r$ and $s$ are even; $K \rightarrow \mathrm{SO}(3)$, if both $r$ and $s$ are odd; $K \rightarrow \mathrm{U}_{2}(\mathbb{C})$, if $r$ and $s$ have different parities. A study of various double covers of $K$ will, in analogy to what we sketched above for diagrams of type $\mathrm{C}_{2}$ and $\mathrm{G}_{2}$, enable us to replace edges labelled $\infty$ by nonedges, simple edges, resp. double edges, thus allowing us to understand the non-two-spherical situation as well. Again, the case in which $r$ and $s$ have different parities lead to some subtleties that we get control of with the concept of admissible colourings introduced in Definition 16.2 .

Following this strategy leads directly to Proposition 17.10 .

Strategy 12.2. Let $\Pi$ with type set $I$ and (generalized) Cartan matrix $A=(a(i, j))_{i, j \in I}$ be an irreducible doubly laced diagram that admits two root lengths. Then the unfolded Dynkin diagram is the simply laced Dynkin diagram $\Pi^{\text {un }}$ with type set

$$
I^{\text {un }}:=\{ \pm i \mid i \in I, i \text { short } \operatorname{root}\} \cup\{i \mid i \in I, i \text { long root }\}
$$

and edges defined via the generalized Cartan matrix $A^{\text {un }}=\left(a^{\text {un }}(i, j)\right)_{i, j \in I^{\text {un }}}$ given by

$$
a^{\mathrm{un}}(i, j)= \begin{cases}0, & \text { if }|i|,|j| \text { have different lengths and } a(|i|,|j|)=0 \\ -1, & \text { if }|i|,|j| \text { have different lengths and } a(|i|,|j|) \neq 0 \\ a(|i|,|j|), & \text { if }|i|,|j| \text { have the same length and } i j>0 \\ 0, & \text { if }|i|,|j| \text { have the same length and } i j<0\end{cases}
$$

(cf. Definition [17.3).

There exists an embedding of $K(\Pi)$ into $K\left(\Pi^{\mathrm{un}}\right)$ that by Corollary 17.6 allows one to related the respective spin covers to one another.

\section{Diagrams of TyPe $\mathrm{G}_{2}$}

In this section we prepare Strategy 12.1 for diagrams of type $\mathrm{G}_{2}$.

Definition 13.1. Denote by $\mathbb{H}:=\{a+b i+c j+d k \mid a, b, c, d \in \mathbb{R}\}$ the real quaternions. Then the standard involution of $\mathbb{H}$ is given by

$$
\ulcorner: \mathbb{H} \rightarrow \mathbb{H}: x=a+b i+c j+d k \mapsto \bar{x}=a-b i-c j-d k
$$

The set of purely imaginary quaternions, cf. $\left[\mathrm{SBG}^{+} 95\right.$, 11.6], is

$$
\mathrm{Pu} \mathbb{H}:=\{x \in \mathbb{H} \mid x=-\bar{x}\}=\{b i+c j+d k \mid b, c, d \in \mathbb{R}\} \subset \mathbb{H} .
$$

Definition 13.2. The split Cayley algebra $\mathbb{O}$ is defined as the vector space $\mathbb{H} \oplus \mathbb{H}$ endowed with the multiplication

$$
x y=\left(x_{1}, x_{2}\right)\left(y_{1}, y_{2}\right)=\left(x_{1} y_{1}+y_{2} \overline{x_{2}}, y_{1} x_{2}+\overline{x_{1}} y_{2}\right),
$$

cf. Coh95, Section 5.1]. The real split Cayley hexagon $\mathcal{H}(\mathbb{R})$ consists of the one- and two-dimensional real subspaces of $\mathbb{O}$ for which the restriction of the multiplication map is trivial, i.e., $\mathcal{H}(\mathbb{R})=(\mathcal{P}, \mathcal{L}, \subset)$ with the point set

$$
\mathcal{P}:=\left\{\langle x\rangle_{\mathbb{R}} \mid x \in \mathbb{O}, x^{2}=0 \neq x\right\}
$$

and the line set

$$
\mathcal{L}:=\left\{\langle x, y\rangle_{\mathbb{R}} \mid\langle x\rangle_{\mathbb{R}} \neq\langle y\rangle_{\mathbb{R}} \in \mathcal{P}, x y=0\right\},
$$

cf. [Coh95, Section 5.1], also [VM98, Section 2.4.9].

Lemma 13.3. Let $x=\left(x_{1}, x_{2}\right) \in \mathbb{O}$. Then $x^{2}=0$ if and only if $x_{1} \in \mathrm{PuHI}$ and $\overline{x_{1}} x_{1}-\overline{x_{2}} x_{2}=0$.

Proof. Suppose $x_{1} \in \mathrm{Pu} \mathbb{H}$ and $\overline{x_{1}} x_{1}-\overline{x_{2}} x_{2}=0$. Then $-x_{1} x_{1}-x_{2} \overline{x_{2}}=\overline{x_{1}} x_{1}-\overline{x_{2}} x_{2}=0$ and $x_{1} x_{2}+\overline{x_{1}} x_{2}=$ $x_{1} x_{2}-x_{1} x_{2}=0$, and, thus, $x^{2}=0$. Conversely, suppose $x^{2}=0$. Then $0=x^{2}=\left(x_{1} x_{1}+x_{2} \overline{x_{2}}, x_{1} x_{2}+\overline{x_{1}} x_{2}\right)$, so if $x_{2}=0$, then $x_{1}=0$, and there is nothing to show. For $x_{2} \neq 0$ multiplication of $x_{1} x_{2}+\overline{x_{1}} x_{2}=0$ from the right with $x_{2}^{-1}$ gives $x_{1}+\overline{x_{1}}=0$ or, equivalently, $x_{1} \in \mathrm{PuH}$. But now $0=x_{1} x_{1}+x_{2} \overline{x_{2}}=-\overline{x_{1}} x_{1}+\overline{x_{2}} x_{2}$, and the claim follows.

Definition 13.4. Let $N: \mathbb{H} \rightarrow \mathbb{R}: x \mapsto x \bar{x}$ be the norm map associated to the standard involution of the real quaternions. Moreover, let

$$
\mathrm{U}_{1}(\mathbb{H}):=\{x \in \mathbb{H} \mid x \bar{x}=1\}
$$

be the group of real quaternions of norm one. 
By Remark 7.3 (see also $\left[\mathrm{SBG}^{+}\right.$95, Lemma 11.22]), the group $\mathrm{SO}(4)$ is isomorphic to the group consisting of the maps

$$
\mathbb{H} \rightarrow \mathbb{H}: x \mapsto a x b^{-1} \quad \text { for } \quad a, b \in \mathrm{U}_{1}(\mathbb{H}) .
$$

Lemma 13.5. The group $\mathrm{SO}(4) \cong\left\{\mathbb{H} \rightarrow \mathbb{H}: x \mapsto a x b^{-1} \mid a, b \in \mathrm{U}_{1}(\mathbb{H})\right\}$ acts flag-transitively on the split Cayley hexagon $\mathcal{H}(\mathbb{R})$ via

$$
\left\langle\left(x_{1}, x_{2}\right)\right\rangle_{\mathbb{R}} \mapsto\left\langle\left(a x_{1} a^{-1}, a x_{2} b^{-1}\right)\right\rangle_{\mathbb{R}}
$$

Proof. We show that the map

$$
f_{a, b}: \mathbb{O} \rightarrow \mathbb{O}:\left(x_{1}, x_{2}\right) \mapsto\left(a x_{1} a^{-1}, a x_{2} b^{-1}\right)
$$

is an algebra automorphism of $\mathbb{O}$ for all $a, b \in \mathrm{U}_{1}(\mathbb{H})$. Then it also induces an automorphism of $\mathcal{H}(\mathbb{R})$, because it is defined via the multiplication in $\mathbb{O}$. We have

$$
\begin{aligned}
& f_{a, b}\left(x_{1}, x_{2}\right) \cdot f_{a, b}\left(y_{1}, y_{2}\right) \\
= & \left(a x_{1} a^{-1}, a x_{2} b^{-1}\right) \cdot\left(a y_{1} a^{-1}, a y_{2} b^{-1}\right) \\
= & \left(\left(a x_{1} a^{-1}\right)\left(a y_{1} a^{-1}\right)+\left(a y_{2} b^{-1}\right) \overline{\left(a x_{2} b^{-1}\right)},\left(a y_{1} a^{-1}\right)\left(a x_{2} b^{-1}\right)+\overline{\left(a x_{1} a^{-1}\right)}\left(a y_{2} b^{-1}\right)\right) \\
= & \left(a\left(x_{1} y_{1}+y_{2} \overline{x_{2}}\right) a^{-1}, a\left(y_{1} x_{2}+\overline{x_{1}} y_{2}\right) b^{-1}\right) \\
= & f_{a, b}\left(\left(x_{1}, x_{2}\right)\left(y_{1}, y_{2}\right)\right),
\end{aligned}
$$

whence the map $f_{a, b}$ is multiplicative. Since it is certainly an $\mathbb{R}$-linear bijection, it is an algebra automorphism of $\mathbb{O}$. Flag-transitivity is an immediate consequence of the Iwasawa decomposition and the fact that $\mathrm{SO}(4)$ is the maximal compact subgroup of the (simply connected semisimple) split real group $\mathrm{G}_{2}(2)$ of type $\mathrm{G}_{2}$.

Remark 13.6. There exists a nice direct proof of flag-transitivity without making use of the Iwasawa decomposition and the structure theory of $\mathrm{G}_{2}(2)$ that in particular illustrates how to compute point and line stabilizers and, thus, helps our understanding how to properly embed the circle group into $\mathrm{SO}(4)$ for our amalgamation problem.

Let

$$
\mathrm{Pu} \mathbb{O}:=\mathrm{Pu} \mathbb{H} \oplus \mathbb{H}
$$

be the set of purely imaginary split octonions and consider the points of the real (projective) quadric

$$
\overline{x_{1}} x_{1}-\overline{x_{2}} x_{2}=0
$$

in $\mathrm{Pu} \mathbb{O}$, i.e., the set of isotropic one dimensional real subspaces of PuOD. By Remark 7.3 (see also $\left[\mathrm{SBG}^{+} 95\right.$, 11.24]), the group $\mathrm{SO}(3)$ is isomorphic to the group consisting of the maps

$$
\mathrm{Pu} \mathbb{H} \rightarrow \mathrm{Pu} \mathbb{H}: x \mapsto a x a^{-1} \text { for } a \in \mathrm{U}_{1}(\mathbb{H})
$$

and acts transitively on the set $\{\{x,-x\} \subset \mathrm{Pu} \mathbb{H} \mid x \bar{x}=1\}$. Moreover, for each $a, x, z \in \mathrm{U}_{1}(\mathbb{H})$, there exists a unique solution $b \in \mathrm{U}_{1}(\mathbb{H})$ for the equation $z=a x b^{-1}$.

Hence $\mathrm{SO}(4)$ acts transitively on the set

$$
\{\{(x, y),(-x,-y)\} \subset \mathrm{Pu} \mathbb{H} \times \mathbb{H} \mid x \bar{x}=1=y \bar{y}\} .
$$

But this implies point transitivity on the projective real quadric $\overline{x_{1}} x_{1}-\overline{x_{2}} x_{2}=0$ in $\mathrm{Pu} \mathbb{O}$, which, in turn, implies point transitivity on $\mathcal{H}(\mathbb{R})$ by Lemma 13.3 .

Now choose one point of $\mathcal{H}(\mathbb{R})$, say $\langle(i, i)\rangle_{\mathbb{R}}$. Then a point $\langle y\rangle_{\mathbb{R}}=\left\langle\left(y_{1}, y_{2}\right)\right\rangle_{\mathbb{R}}$ is collinear to this point if and only if

$$
\left(i y_{1}-y_{2} i, y_{1} i-i y_{2}\right)=0 \Longleftrightarrow y_{1}=-i y_{2} i .
$$

So the question of transitivity of the stabilizer of $\langle(i, i)\rangle_{\mathbb{R}}$ in $\mathrm{SO}(4)$ on the line pencil of $\langle(i, i)\rangle_{\mathbb{R}}$ in $\mathcal{H}(\mathbb{R})$ is equivalent to the question of transitivity of the stabilizer of $\langle i\rangle_{\mathbb{R}}$ in $\mathrm{SO}(3)$ on the line pencil of $\langle i\rangle_{\mathbb{R}}$ in the projective plane $\mathrm{Pu} \mathbb{H} \cong \mathrm{Pu} \mathbb{H} \oplus\{0\} \subset \mathrm{Pu} \mathbb{O}$.

But since the latter is transitive, so is the former, and hence $\mathrm{SO}(4)$ acts flag-transitively on $\mathcal{H}(\mathbb{R})$ by means of the maps given in Lemma 13.5.

The stabilizer of the point $\langle(i, i)\rangle_{\mathbb{R}}$ contains the circle group $\mathrm{SO}(2)$ acting naturally diagonally on $\left\langle j_{1}, k_{1}\right\rangle_{\mathbb{R}} \oplus\left\langle j_{2}, k_{2}\right\rangle_{\mathbb{R}}$. The stabilizer of the line $\langle(i, i),(j,-j)\rangle_{\mathbb{R}}$ in $\mathrm{SO}(4)$ contains $\mathrm{SO}(2)$ acting naturally by rotations on $\left\langle i_{1}, j_{1}\right\rangle_{\mathbb{R}}$ and by rotations in the opposite direction on $\left\langle i_{2}, j_{2}\right\rangle_{\mathbb{R}}$ in such a way that an element with first coordinate $\lambda i_{1}+\mu j_{1}$ has second coordinate $\lambda i_{2}-\mu j_{2}$. 
Notation 13.7. We denote these embeddings of the circle group into $\mathrm{SO}(4)$ by $\eta_{p}$ resp. $\eta_{l}$. Concretely, one has

$$
\begin{aligned}
& \eta_{p}: \mathrm{SO}(2) \rightarrow \mathrm{SO}(4): D(\alpha) \mapsto\left(\begin{array}{ll}
{ }^{I_{2}} & \\
& D(\alpha)
\end{array}\right)=\varepsilon_{34}(D(\alpha)),
\end{aligned}
$$

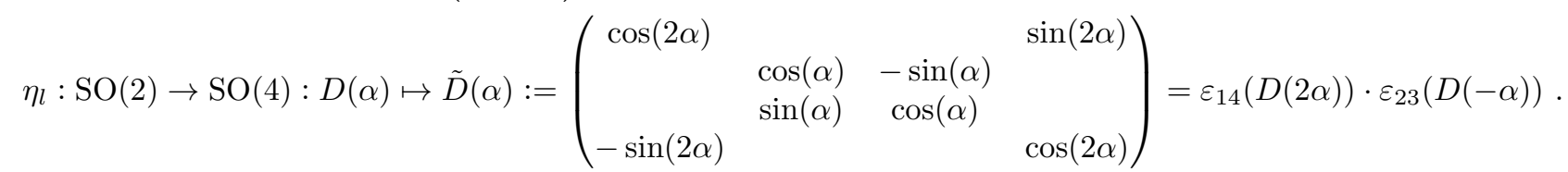

Lemma 13.8. Let $B:=\operatorname{diag}(-1,1,1,-1), C:=\operatorname{diag}(-1,-1,1,1) \in \mathrm{U}_{2}(\mathbb{C})$. Then the following hold:

(a) The map $\gamma_{B}: \mathrm{SO}(4) \rightarrow \mathrm{SO}(4): A \mapsto B \cdot A \cdot B^{-1}=B \cdot A \cdot B$ is an automorphism of $\mathrm{SO}(4)$ such that

$$
\gamma_{B} \circ \eta_{p}=\eta_{p} \circ \text { inv } \quad \text { and } \quad \gamma_{B} \circ \eta_{l}=\eta_{l}
$$

(b) The map $\gamma_{C}: \mathrm{SO}(4) \rightarrow \mathrm{SO}(4): A \mapsto C \cdot A \cdot C^{-1}=C \cdot A \cdot C$ is an automorphism of $\mathrm{SO}(4)$ such that

$$
\gamma_{C} \circ \eta_{p}=\eta_{p} \quad \text { and } \quad \gamma_{C} \circ \eta_{l}=\eta_{l} \circ \text { inv } .
$$

Proof. Straightforward.

Notation 13.9. In the following, let

$$
\begin{aligned}
& \tilde{\eta}_{p}: \operatorname{Spin}(2) \rightarrow \operatorname{Spin}(4): S(\alpha) \mapsto \tilde{\varepsilon}_{34}(S(\alpha)), \\
& \tilde{\eta}_{l}: \operatorname{Spin}(2) \rightarrow \operatorname{Spin}(4): S(\alpha) \mapsto \tilde{\varepsilon}_{14}(S(2 \alpha)) \cdot \tilde{\varepsilon}_{23}(S(-\alpha)),
\end{aligned}
$$

and recall from Theorem 6.8(b) that for $n \geq 2$ the map

$$
\rho_{n}: \operatorname{Spin}(n) \rightarrow \operatorname{SO}(n)
$$

is the twisted adjoint representation.

In order to generalize our definition of spin amalgams, we need $\tilde{\eta}_{p}$ and $\tilde{\eta}_{l}$ to be injective. For the former this is clear from its definition, for the latter we verify it now.

Lemma 13.10. The map $\tilde{\eta}_{l}$ is a monomorphism.

Proof. For $S(\alpha) \in \operatorname{ker} \tilde{\eta}_{l}$ one has

$$
\tilde{\eta}_{l}(S(\alpha))=\left(\cos (2 \alpha)+\sin (2 \alpha) e_{1} e_{4}\right)\left(\cos (\alpha)-\sin (\alpha) e_{2} e_{3}\right)=1
$$

and, thus, $\alpha \in \pi \mathbb{Z}$. As $\tilde{\eta}_{l}(S(\pi))=-1$ and $\tilde{\eta}_{l}(S(2 \pi))=1$, one obtains

$$
\operatorname{ker} \tilde{\eta}_{l}=\{S(\alpha) \mid \alpha \in 2 \pi \mathbb{Z}\}=\{1\} \text {. }
$$

Lemma 13.11. One has

$$
\rho_{4} \circ \tilde{\eta}_{p}=\eta_{p} \circ \rho_{2}, \quad \rho_{4} \circ \tilde{\eta}_{l}=\eta_{l} \circ \rho_{2} .
$$

Proof. For $\alpha \in \mathbb{R}$,

$$
\left(\rho_{4} \circ \tilde{\eta}_{p}\right)(S(\alpha))=\left(\rho_{4} \circ \tilde{\varepsilon}_{34}\right)(S(\alpha))=\left(\varepsilon_{34} \circ \rho\right)(S(\alpha))=\left(\eta_{p} \circ \rho\right)(S(\alpha))
$$

and

$$
\begin{aligned}
\left(\rho_{4} \circ \tilde{\eta}_{l}\right)(S(\alpha)) & =\rho_{4}\left(\tilde{\varepsilon}_{14}(S(2 \alpha)) \cdot \tilde{\varepsilon}_{23}(S(-\alpha))\right)=\varepsilon_{14}(D(4 \alpha)) \cdot \varepsilon_{23}(D(-2 \alpha)) \\
& =\left(\eta_{l} \circ \rho_{2}\right)(S(\alpha)) .
\end{aligned}
$$

Lemma 13.12. Let $V:=\mathbb{H}$ and $\mathcal{E}:=\{1, i, j, k\}$. Then the following hold:

(a) For $a, b \in \mathrm{U}_{1}(\mathbb{H})$ the maps

$$
\ell_{a}: \mathbb{H} \rightarrow \mathbb{H}: x \mapsto \text { ax and } r_{b}: \mathbb{H} \rightarrow \mathbb{H}: x \mapsto x b^{-1}
$$

preserve the norm $N: \mathbb{H} \rightarrow \mathbb{R}: x \mapsto x \bar{x}$. In particular, $\ell_{a}, r_{b} \in \mathrm{SO}(\mathbb{H}) \cong \mathrm{SO}(4)$.

(b) The map

$$
\mathrm{U}_{1}(\mathbb{H}) \times \mathrm{U}_{1}(\mathbb{H}) \rightarrow \mathrm{SO}(4):(a, b) \mapsto \ell_{a} \circ r_{b}
$$

is a group epimorphism with kernel $\{(1,1),(-1,-1)\}$.

Proof. This has been discussed in Remark 7.3. Alternatively, it also follows from $\mathrm{SBG}^{+}$95, Lemma 11.22 to Corollary 11.25]. 
For $\mathcal{E}=\{1, i, j, k\}$ define

$$
L_{a}:=M_{\mathcal{E}}\left(\ell_{a}\right) \in \mathrm{SO}(4), \quad R_{b}:=M_{\mathcal{E}}\left(r_{b}\right) \in \mathrm{SO}(4) .
$$

Remark 13.13. (a) The map $\mathrm{U}_{1}(\mathbb{H}) \times \mathrm{U}_{1}(\mathbb{H}) \rightarrow \mathrm{SO}(4)$ from Lemma $13.12(\mathrm{~b})$ equals the covering map $\rho_{4}$, cf. Remark 7.4 .

(b) Given $x=a+b i+c j+d k \in \mathrm{U}_{1}(\mathbb{H})$, a short computation shows

$$
L_{x}=\left(\begin{array}{cccc}
a & -b & -c & -d \\
b & a & -d & c \\
c & d & a & -b \\
d & -c & b & a
\end{array}\right), \quad R_{x}=\left(\begin{array}{cccc}
a & b & c & d \\
-b & a & -d & c \\
-c & d & a & -b \\
-d & -c & b & a
\end{array}\right)
$$

as $\mathbb{R}$-linear maps via left action. Lemma $13.12(\mathrm{~b})$ implies that for all $x, y \in \mathrm{U}_{1}(\mathbb{H})$ one has $L_{x} R_{y}=R_{y} L_{x}$ and that up to scalar multiplication with -1 the matrices $L_{x}$ and $R_{y}$ are uniquely determined by their product.

(c) The action from Lemma 13.5 translates into

$$
\omega: \mathrm{SO}(4) \times \mathcal{H}(\mathbb{R}) \rightarrow \mathcal{H}(\mathbb{R}):\left(L_{a} R_{b},(x, y)\right) \mapsto\left(L_{a} R_{a} \cdot x, L_{a} R_{b} \cdot y\right) .
$$

(d) For $\alpha \in \mathbb{R}$, one has $\eta_{p}(D(\alpha))=L_{a} \cdot R_{a}$ with $a=\cos \left(\frac{\alpha}{2}\right)-\sin \left(\frac{\alpha}{2}\right) i$, i.e.,

$$
\left(\begin{array}{ll}
I_{2} & \\
& D(\alpha)
\end{array}\right)=\left(\begin{array}{ll}
D\left(\frac{\alpha}{2}\right) & \\
& D\left(\frac{\alpha}{2}\right)
\end{array}\right) \cdot\left(\begin{array}{ll}
D\left(-\frac{\alpha}{2}\right) & \\
& D\left(\frac{\alpha}{2}\right)
\end{array}\right) .
$$

(e) For $\alpha \in \mathbb{R}$, we have $\eta_{l}(D(\alpha))=L_{a} \cdot R_{b}$ with $a=\cos \left(\frac{\alpha}{2}\right)-\sin \left(\frac{\alpha}{2}\right) k$ and $b=\cos \left(\frac{3 \alpha}{2}\right)+\sin \left(\frac{3 \alpha}{2}\right) k$, i.e.,

$$
\eta_{l}(D(\alpha))=\left(\begin{array}{ccc}
\cos \left(\frac{\alpha}{2}\right) & & \sin \left(\frac{\alpha}{2}\right) \\
-\sin \left(\frac{\alpha}{2}\right) & D\left(\frac{\alpha}{2}\right) & \cos \left(\frac{\alpha}{2}\right)
\end{array}\right) \cdot\left(\begin{array}{ccc}
\cos \left(\frac{3 \alpha}{2}\right) & & \sin \left(\frac{3 \alpha}{2}\right) \\
-\sin \left(\frac{3 \alpha}{2}\right) & D\left(-\frac{3 \alpha}{2}\right) & \cos \left(\frac{3 \alpha}{2}\right)
\end{array}\right) .
$$

The subgroup of right translations $R_{b}$ is normal in $\mathrm{SO}(4)$, the resulting quotient is isomorphic to $\mathrm{SO}(3)$. This canonical projection induces a surjection from the split Cayley hexagon onto the real projective plane, given by the projection $\left\langle\left(x_{1}, x_{2}\right)\right\rangle_{\mathbb{R}} \mapsto\left\langle x_{1}\right\rangle_{\mathbb{R}}$. (Cf. [Gra98, Section 5]. An alternative description of this surjection can be found in GV00.)

The following lemma describes how the corresponding embedded circle groups behave under this surjection.

Lemma 13.14. (a) There is an epimorphism $\eta_{1}: \mathrm{SO}(4) \rightarrow \mathrm{SO}(3)$ such that

$$
\eta_{1} \circ \eta_{p}=\varepsilon_{23}, \quad \eta_{1} \circ \eta_{l}=\varepsilon_{12} \text {. }
$$

(b) There is an epimorphism $\eta_{2}: \mathrm{SO}(4) \rightarrow \mathrm{SO}(3)$ such that

$$
\eta_{2} \circ \eta_{p}=\varepsilon_{12}, \quad \eta_{2} \circ \eta_{l}=\varepsilon_{23} .
$$

Proof. By Remark 7.3, the map

$$
\psi: \mathrm{SO}(4) \rightarrow \varepsilon_{\{2,3,4\}}(\mathrm{SO}(3)): L_{a} R_{b} \mapsto L_{a} R_{a}
$$

is an epimorphism (see also $\mathrm{SBG}^{+}$95, Corollaries 11.23 and 11.24]). By Remark $13.13(\mathrm{~d})$,

and, by Remark $13.13(\mathrm{e})$

$$
\left(\psi \circ \eta_{l}\right)(D(\alpha))=\left(\begin{array}{ccc}
\cos \left(\frac{\alpha}{2}\right) & & \sin \left(\frac{\alpha}{2}\right) \\
-\sin \left(\frac{\alpha}{2}\right) & D\left(\frac{\alpha}{2}\right) & \cos \left(\frac{\alpha}{2}\right)
\end{array}\right)\left(\begin{array}{ccc}
\cos \left(\frac{\alpha}{2}\right) & & -\sin \left(\frac{\alpha}{2}\right) \\
\sin \left(\frac{\alpha}{2}\right) & D\left(\frac{\alpha}{2}\right) & \cos \left(\frac{\alpha}{2}\right)
\end{array}\right)=\left(\begin{array}{lll}
1 & & \\
& D(\alpha) & \\
& & 1
\end{array}\right)=\varepsilon_{23}(D(\alpha)) .
$$

Therefore, the map $\eta_{1}:=\varepsilon_{\{2,3,4\}}^{-1} \circ \psi$ has the desired properties. The existence of the map $\eta_{2}$ now follows from Lemma 9.4

The final result of this section allows us to carry out Strategy 12.1 for edges of type $\mathrm{G}_{2}$ in Theorem 17.10 below.

Proposition 13.15. $\quad$ (a) There is an epimorphism $\tilde{\eta}_{1}: \operatorname{Spin}(4) \rightarrow \operatorname{Spin}(3)$ such that

$$
\tilde{\eta}_{1} \circ \tilde{\eta}_{p}=\tilde{\varepsilon}_{23}, \quad \tilde{\eta}_{1} \circ \tilde{\eta}_{l}=\tilde{\varepsilon}_{12} .
$$

(b) There is an epimorphism $\tilde{\eta}_{2}: \operatorname{Spin}(4) \rightarrow \operatorname{Spin}(3)$ such that

$$
\tilde{\eta}_{2} \circ \tilde{\eta}_{p}=\tilde{\varepsilon}_{12}, \quad \tilde{\eta}_{2} \circ \tilde{\eta}_{l}=\tilde{\varepsilon}_{23} .
$$


Proof. The map $\tilde{\eta}_{1}: \operatorname{Spin}(4) \rightarrow \operatorname{Spin}(3): u+\mathbb{I} v \mapsto u+v$ makes the inner/right-hand quadrangle of the following diagram commute (see Remarks 7.2 and 7.4 ):

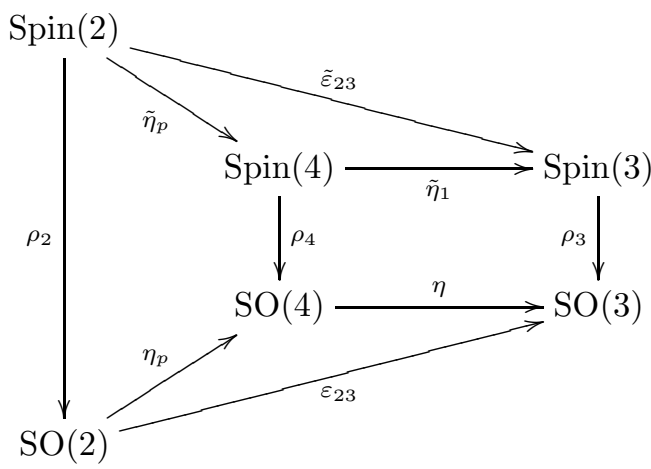

The lower triangle commutes by Lemma 13.14. The left-hand quadrangle commutes by Lemma 13.11, Hence

and

$$
\varepsilon_{23} \circ \rho_{2}=\eta \circ \eta_{p} \circ \rho_{2}=\eta \circ \rho_{4} \circ \tilde{\eta}_{p}=\rho_{3} \circ \tilde{\eta}_{1} \circ \tilde{\eta}_{p}
$$

$$
\operatorname{ker}\left(\rho_{3} \circ \tilde{\eta}_{1} \circ \tilde{\eta}_{p}\right)=\operatorname{ker}\left(\varepsilon_{23} \circ \rho_{2}\right)=\operatorname{ker} \rho_{2}=\left\{ \pm 1_{\operatorname{Spin}(2)}\right\} .
$$

Therefore $\tilde{\varepsilon}_{23}=\tilde{\eta}_{1} \circ \tilde{\eta}_{p}$ by Proposition 8.5 . In particular, also the upper triangle of the diagram commutes.

The second claim concerning $\tilde{\eta}_{1}$ follows by an analogous argument

The claims concerning $\tilde{\eta}_{2}$ are now immediate by Lemma 9.4 and Proposition 8.4

\section{Diagrams of type $\mathrm{C}_{2}$}

In this section we prepare Strategy 12.1 for diagrams of type $\mathrm{C}_{2}$.

Definition 14.1. Let $\mathrm{Sp}_{4}(\mathbb{R})$ be the matrix group with respect to the $\mathbb{R}$-basis $e_{1}, i e_{1}, e_{2}, i e_{2}$ of $\mathbb{C}^{2}$ leaving the real alternating form

$$
\left(\left(x_{1}, x_{2}, x_{3}, x_{4}\right),\left(y_{1}, y_{2}, y_{3}, y_{4}\right)\right) \mapsto x_{1} y_{2}-x_{2} y_{1}+x_{3} y_{4}-x_{4} y_{3}
$$

invariant.

Remark 14.2. The maximal compact subgroup of $\mathrm{Sp}_{4}(\mathbb{R})$ is the group $\mathrm{U}_{2}(\mathbb{C})$, embedded as follows. Let $e_{1}, e_{2}$ be the standard basis of $\mathbb{C}^{2}$ and consider $\mathrm{U}_{2}(\mathbb{C})$ as the isometry group of the scalar product $\left(\left(v_{1}, v_{2}\right),\left(w_{1}, w_{2}\right)\right) \mapsto \overline{v_{1}} w_{1}+\overline{v_{2}} w_{2}$. Defining

$$
\begin{array}{ll}
x_{1}:=\operatorname{Re}\left(v_{1}\right), & x_{2}:=\operatorname{Im}\left(v_{1}\right), \\
x_{3}:=\operatorname{Re}\left(v_{2}\right), & x_{4}:=\operatorname{Im}\left(v_{2}\right), \\
y_{1}:=\operatorname{Re}\left(w_{1}\right), & y_{2}:=\operatorname{Im}\left(w_{1}\right), \\
y_{3}:=\operatorname{Re}\left(w_{2}\right), & y_{4}:=\operatorname{Im}\left(w_{2}\right),
\end{array}
$$

we compute

$$
\overline{v_{1}} w_{1}+\overline{v_{2}} w_{2}=x_{1} y_{1}+x_{2} y_{2}+x_{3} y_{3}+x_{4} y_{4}+i\left(x_{1} y_{2}-x_{2} y_{1}+x_{3} y_{4}-x_{4} y_{3}\right) .
$$

Since two complex numbers are equal if and only if real part and imaginary part coincide, the group $\mathrm{U}_{2}(\mathbb{C})$ preserves the form $x_{1} y_{2}-x_{2} y_{1}+x_{3} y_{4}-x_{4} y_{3}$ and we have found an embedding in $\mathrm{Sp}_{4}(\mathbb{R})$, acting naturally on the $\mathbb{R}$-vector space $\mathbb{C}^{2}$ with $\mathbb{R}$-basis $e_{1}, i e_{1}, e_{2}, i e_{2}$.

As $\mathrm{U}_{2}(\mathbb{C})$ also preserves the form $x_{1} y_{1}+x_{2} y_{2}+x_{3} y_{3}+x_{4} y_{4}$ with respect to the $\mathbb{R}$-basis $e_{1}, i e_{1}, e_{2}$, $i e_{2}$ of $\mathbb{C}^{2}$ it is at the same time also a subgroup of $\mathrm{O}(4)$, in fact of $\mathrm{SO}(4)$, since $\mathrm{U}_{2}(\mathbb{C})$ is connected with respect to its Lie group topology. We will give concrete coordinates for this embedding in Remark 14.7 below.

The real symplectic quadrangle can be modelled as the point-line geometry consisting of the onedimensional and two-dimensional subspaces of $\mathbb{R}^{4}$ which are totally isotropic with respect to a nondegenerate alternating bilinear form, cf. [VM98, Section 2.3.17], also [BC13, Section 10.1].

Using the $\mathbb{R}$-basis $e_{1}, i e_{1}, e_{2}, i e_{2}$ and the $\mathbb{R}$-alternating form on $\mathbb{C}^{2}$ given in Remark 14.2 , an incident point-line pair of the resulting symplectic quadrangle of $\operatorname{Sp}_{4}(\mathbb{R})$ is given by $\left\langle e_{1}\right\rangle_{\mathbb{R}} \subset\left\langle e_{1}, e_{2}\right\rangle_{\mathbb{R}}$. The stabilizer in $\mathrm{U}_{2}(\mathbb{C})$ of the point $\left\langle e_{1}\right\rangle_{\mathbb{R}}$ is isomorphic to $\mathrm{O}_{1}(\mathbb{R}) \times \mathrm{U}_{1}(\mathbb{C})$ where the first factor acts diagonally on $\left\langle e_{1}\right\rangle_{\mathbb{R}} \oplus\left\langle i e_{1}\right\rangle_{\mathbb{R}}$ and the second factor acts naturally on $\left\langle e_{2}\right\rangle_{\mathbb{C}}$. The stabilizer of the line $\left\langle e_{1}, e_{2}\right\rangle_{\mathbb{R}}$ is isomorphic to $\mathrm{O}_{2}(\mathbb{R})$ acting diagonally on $\left\langle e_{1}, e_{2}\right\rangle_{\mathbb{R}} \oplus\left\langle i e_{1}, i e_{2}\right\rangle_{\mathbb{R}}$. 
Definition 14.3. Let

$$
\zeta_{p}: \mathrm{SO}(2) \rightarrow \mathrm{U}_{2}(\mathbb{C}) \subset \mathrm{Sp}_{4}(\mathbb{R}) \cap \mathrm{SO}(4):\left(\begin{array}{cc}
x & y \\
-y & x
\end{array}\right) \mapsto\left(\begin{array}{cccc}
1 & & & \\
& 1 & & \\
& & x & y \\
& & -y & x
\end{array}\right)
$$

and let

$$
\zeta_{l}: \mathrm{SO}(2) \rightarrow \mathrm{U}_{2}(\mathbb{C}) \subset \mathrm{Sp}_{4}(\mathbb{R}) \cap \mathrm{SO}(4):\left(\begin{array}{cc}
x & y \\
-y & x
\end{array}\right) \mapsto\left(\begin{array}{cccc}
x & & y & \\
& x & & y \\
-y & & x & \\
& -y & & x
\end{array}\right)
$$

be the embeddings of the circle group arising as point-stabilizing resp. line-stabilizing rank one groups as above with respect to the $\mathbb{R}$-bases $e_{1}, e_{2}$ of $\mathbb{R}^{2}$ and $e_{1}, i e_{1}, e_{2}, i e_{2}$ of $\mathbb{C}^{2}$.

Recall the definitions of $D(\alpha)$ and $S(\alpha)$ from Notation 8.1 ,

Notation 14.4. In the following, identify $\mathbb{C}=\{x+i y \mid x, y \in \mathbb{R}\}$ with $\left\{\left(\begin{array}{cc}x & y \\ -y & x\end{array}\right) \mid x, y \in \mathbb{R}\right\}$. This identification, in particular, embeds $\mathrm{SO}(2)$ into $\mathbb{C}$ as the unit circle group. For $\alpha \in \mathbb{R}$, let

$$
\tilde{D}(\alpha):=\zeta_{l}(D(\alpha))=\left(\begin{array}{cccc}
\cos (\alpha) & & \sin (\alpha) & \\
& \cos (\alpha) & & \sin (\alpha) \\
-\sin (\alpha) & & \cos (\alpha) & \\
& -\sin (\alpha) & & \cos (\alpha)
\end{array}\right) \in \mathrm{U}_{2}(\mathbb{C}) . .
$$

Lemma 14.5. Let $B:=\operatorname{diag}(-1,1,-1,1), C:=\operatorname{diag}(-1,-1,1,1) \in \mathrm{U}_{2}(\mathbb{C})$. Then the following hold:

(a) The map $\gamma_{B}: \mathrm{U}_{2}(\mathbb{C}) \rightarrow \mathrm{U}_{2}(\mathbb{C}): A \mapsto B \cdot A \cdot B^{-1}=B \cdot A \cdot B$ is an automorphism of $\mathrm{U}_{2}(\mathbb{C})$ such that

$$
\gamma_{B} \circ \zeta_{p}=\zeta_{p} \circ \text { inv } \quad \text { and } \quad \gamma_{B} \circ \zeta_{l}=\zeta_{l} .
$$

(b) The map $\gamma_{C}: \mathrm{U}_{2}(\mathbb{C}) \rightarrow \mathrm{U}_{2}(\mathbb{C}): A \mapsto C \cdot A \cdot C^{-1}=C \cdot A \cdot C$ is an automorphism of $\mathrm{U}_{2}(\mathbb{C})$ such that

$$
\gamma_{C} \circ \zeta_{p}=\zeta_{p} \quad \text { and } \quad \gamma_{C} \circ \zeta_{l}=\zeta_{l} \circ \text { inv }
$$

Proof. Straightforward.

Lemma 14.6. Let $\alpha \in \mathbb{R}$. Then $\tilde{D}(\alpha) \in \mathrm{SU}_{2}(\mathbb{C})$ and $\left(\begin{array}{ll}D(-\alpha) & \\ & D(\alpha)\end{array}\right) \in \mathrm{SU}_{2}(\mathbb{C})$.

Proof. Given $\alpha \in \mathbb{R}$, one has the following (where the determinant is taken in $\mathrm{SL}_{2}(\mathbb{C})$ ):

and

$$
\operatorname{det}(\tilde{D}(\alpha))=\left(\begin{array}{ll}
\cos (\alpha) & \\
& \cos (\alpha)
\end{array}\right)^{2}+\left(\begin{array}{ll}
\sin (\alpha) & \\
& \sin (\alpha)
\end{array}\right)^{2}=\left(\begin{array}{ll}
\cos (\alpha)^{2}+\sin (\alpha)^{2} & \\
& \cos (\alpha)^{2}+\sin (\alpha)^{2}
\end{array}\right)=I_{2}=1_{\mathbb{C}}
$$

$$
\operatorname{det}\left(\begin{array}{cc}
D(-\alpha) & \\
& D(\alpha)
\end{array}\right)=D(-\alpha) \cdot D(\alpha)=I_{2}=1_{\mathbb{C}} .
$$

Remark 14.7. Returning to the embedding $\mathrm{U}_{2}(\mathbb{C}) \rightarrow \mathrm{SO}(4)$ mentioned in Remark 14.2, the group

$$
\mathrm{SU}_{2}(\mathbb{C})=\left\{\left(\begin{array}{cc}
x_{1}+i y_{1} & x_{2}+i y_{2} \\
-x_{2}+i y_{2} & x_{1}-i y_{2}
\end{array}\right) \mid x_{1}, x_{2}, y_{1}, y_{2} \in \mathbb{R}, x_{1}^{2}+x_{2}^{2}+y_{1}^{2}+y_{2}^{2}=1\right\} \leq \mathrm{U}_{2}(\mathbb{C})
$$

acts $\mathbb{R}$-linearly on $\mathbb{C}^{2}$ with transformation matrices

$$
\left(\begin{array}{cccc}
x_{1} & -y_{1} & x_{2} & -y_{2} \\
y_{1} & x_{1} & y_{2} & x_{2} \\
-x_{2} & -y_{2} & x_{1} & y_{1} \\
y_{2} & -x_{2} & -y_{1} & x_{1}
\end{array}\right)
$$

with respect to the basis $e_{1}, i e_{1}, e_{2}, i e_{2}$. Remark 13.13 implies that the map

$$
\begin{aligned}
\mathrm{SU}_{2}(\mathbb{C}) & \rightarrow \mathrm{SO}(4) \\
\left(\begin{array}{cc}
x_{1}+i y_{1} & x_{2}+i y_{2} \\
-x_{2}+i y_{2} & x_{1}-i y_{2}
\end{array}\right) & \mapsto \quad R_{x_{1}-y_{1} i+x_{2} j-y_{2} k}=\left(\begin{array}{cccc}
x_{1} & -y_{1} & x_{2} & -y_{2} \\
y_{1} & x_{1} & y_{2} & x_{2} \\
-x_{2} & -y_{2} & x_{1} & y_{1} \\
y_{2} & -x_{2} & -y_{1} & x_{1}
\end{array}\right)
\end{aligned}
$$

injects $\mathrm{SU}_{2}(\mathbb{C})$ into $\mathrm{SO}(4)$. The restriction of this map to $\mathrm{SO}(2) \subset \mathrm{SU}_{2}(\mathbb{C})$ by setting $y_{1}=0=y_{2}$ provides the transformations $\tilde{D}$ from Notation 14.4. The group

$$
\left\{\left(\begin{array}{cc}
\cos (\alpha)+i \sin (\alpha) & 0 \\
0 & \cos (\alpha)+i \sin (\alpha)
\end{array}\right)\right\} \cong \mathrm{U}_{1}(\mathbb{C}) \cong \mathrm{SO}(2)
$$


acts with transformation matrices

$$
\left(\begin{array}{cc}
D(-\alpha) & 0 \\
0 & D(-\alpha)
\end{array}\right)=\left(\begin{array}{cccc}
\cos (\alpha) & -\sin (\alpha) & 0 & 0 \\
\sin (\alpha) & \cos (\alpha) & 0 & 0 \\
0 & 0 & \cos (\alpha) & -\sin (\alpha) \\
0 & 0 & \sin (\alpha) & \cos (\alpha)
\end{array}\right)
$$

i.e., Remark 13.13 implies that the map

$$
\begin{aligned}
& \mathrm{U}_{1}(\mathbb{C}) \rightarrow \mathrm{SO}(4) \\
& \left(\begin{array}{cc}
\cos (\alpha)+i \sin (\alpha) & 0 \\
0 & \cos (\alpha)+i \sin (\alpha)
\end{array}\right) \mapsto L_{\cos (\alpha)+i \sin (\alpha)}=\left(\begin{array}{cccc}
\cos (\alpha) & -\sin (\alpha) & 0 & 0 \\
\sin (\alpha) & \cos (\alpha) & 0 & 0 \\
0 & 0 & \cos (\alpha) & -\sin (\alpha) \\
0 & 0 & \sin (\alpha) & \cos (\alpha)
\end{array}\right)
\end{aligned}
$$

injects $\mathrm{U}_{1}(\mathbb{C})$ into $\mathrm{SO}(4)$. Altogether, using Remark 7.2, we obtain the following commutative diagram:

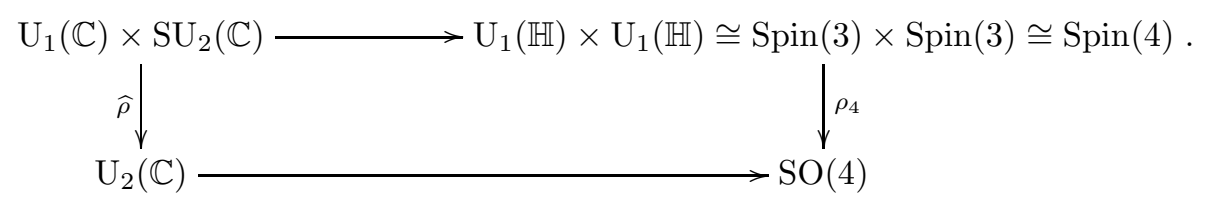

Our candidate for a spin cover of the group $\mathrm{U}_{2}(\mathbb{C})$ therefore is its double cover

$$
\mathrm{U}_{1}(\mathbb{C}) \times \mathrm{SU}_{2}(\mathbb{C}) \rightarrow \mathrm{U}_{2}(\mathbb{C}):(z, A) \mapsto z A .
$$

Note that the fundamental group of $\mathrm{U}_{2}(\mathbb{C})$ equals $\mathbb{Z}$, as the determinant map det $: \mathrm{U}_{2}(\mathbb{C}) \rightarrow \mathrm{U}_{1}(\mathbb{C}) \cong$ $\mathrm{SO}(2)$ induces an isomorphism of fundamental groups; its simply connected universal cover is isomorphic to $\mathbb{R} \times \mathrm{SU}_{2}(\mathbb{C})$. The above double cover is unique up to isomorphism, because $\mathbb{Z}$ has a unique subgroup of index two (cf. [Hat02, Theorem 1.38]).

Notation 14.8. In the following, let

$$
\begin{aligned}
& \tilde{\zeta}_{p}: \operatorname{Spin}(2) \rightarrow \mathrm{SO}(2) \times \mathrm{SU}_{2}(\mathbb{C}) \subset \operatorname{Spin}(4): S(\alpha) \mapsto\left(D(\alpha),\left(\begin{array}{ll}
D(-\alpha) & \\
& D(\alpha)
\end{array}\right)\right), \\
& \tilde{\zeta}_{l}: \operatorname{Spin}(2) \rightarrow \mathrm{SO}(2) \times \mathrm{SU}_{2}(\mathbb{C}) \subset \operatorname{Spin}(4): S(\alpha) \mapsto(\mathrm{id}, \tilde{D}(\alpha))
\end{aligned}
$$

and let

$$
\widehat{\rho}: \mathrm{SO}(2) \times \mathrm{SU}_{2}(\mathbb{C}) \rightarrow \mathrm{U}_{2}(\mathbb{C}):(z, A) \mapsto\left({ }^{z} z\right) \cdot A
$$

Recall the maps

$$
\begin{gathered}
\rho_{2}: \operatorname{Spin}(2) \rightarrow \operatorname{SO}(2): S(\alpha) \mapsto D(2 \alpha), \\
\operatorname{sq}: G \rightarrow G: x \mapsto x^{2}, \\
\operatorname{inv}: G \rightarrow G: x \mapsto x^{-1} .
\end{gathered}
$$

Lemma 14.9. One has

$$
\tilde{\zeta}_{p}=\tilde{\varepsilon}_{34} \circ \mathrm{sq}=\tilde{\varepsilon}_{23} \cdot \tilde{\varepsilon}_{14}
$$

Proof. Using the identification $\operatorname{Spin}(4) \cong \operatorname{Spin}(3) \times \operatorname{Spin}(3)$ and considering the left hand factor as transformations by left multiplication and the right hand factor as transformations by right multiplication of unit quaternions we compute

$$
\begin{aligned}
& \tilde{\varepsilon}_{34}\left(\cos (\alpha)+\sin (\alpha) e_{1} e_{2}\right)=\cos (\alpha)+\sin (\alpha) e_{3} e_{4} \\
& =\cos (\alpha)-\mathbb{I} i \sin (\alpha) \\
& =(\cos (\alpha)-i \sin (\alpha), \cos (\alpha)+i \sin (\alpha)) \\
& =\left(L_{\cos (\alpha)-i \sin (\alpha)}, R_{\cos (\alpha)-i \sin (\alpha)}\right) \\
& =\left(D(\alpha),\left(\begin{array}{ll}
D(-\alpha) & \\
& D(\alpha)
\end{array}\right)\right) \\
& =\tilde{\zeta}_{p}\left(\cos (\alpha)+\sin (\alpha) e_{1} e_{2}\right)
\end{aligned}
$$

by Remark 7.2

by Remark 7.2

by Lemma 13.12, Remark 13.13

by Remark 14.7 
and

$$
\begin{array}{rlr} 
& \tilde{\varepsilon}_{23}\left(\cos (\alpha)+\sin (\alpha) e_{1} e_{2}\right) \tilde{\varepsilon}_{14}\left(\cos (\alpha)+\sin (\alpha) e_{1} e_{2}\right) & \\
= & \left(\cos (\alpha)+\sin (\alpha) e_{2} e_{3}\right)\left(\cos (\alpha)+\sin (\alpha) e_{1} e_{4}\right) & \\
= & (\cos (\alpha))^{2}+j \cos (\alpha) \sin (\alpha)+\mathbb{I}\left((\sin (\alpha))^{2}-j \cos (\alpha) \sin (\alpha)\right) & \text { by Remark } 7.2 \\
= & \left(1,(\cos (\alpha)+j \sin (\alpha))^{2}\right) & \text { by Remark } 7.2 \\
= & & \text { by Lemma 13.12, Remark } 13.13 \\
= & & \text { by Remark } 14.7 \\
= & &
\end{array}
$$

The following observation is the analog of Lemma 13.11 .

Lemma 14.10. One has

$$
\widehat{\rho} \circ \tilde{\zeta}_{p}=\zeta_{p} \circ \rho_{2}, \quad \hat{\rho} \circ \tilde{\zeta}_{l} \circ \mathrm{sq}=\hat{\rho} \circ \mathrm{sq} \circ \tilde{\zeta}_{l}=\zeta_{l} \circ \rho_{2} .
$$

Moreover,

$$
\begin{aligned}
& (\widehat{\rho})^{-1}\left(\zeta_{p}\right)(\mathrm{SO}(2)) \cong \operatorname{Spin}(2) \quad \text { and } \\
& (\widehat{\rho})^{-1}\left(\zeta_{l}\right)(\mathrm{SO}(2)) \cong\{1,-1\} \times \operatorname{SO}(2) .
\end{aligned}
$$

Proof. For $\alpha \in \mathbb{R}$,

$$
\left(\widehat{\rho} \circ \tilde{\zeta}_{p}\right)(S(\alpha))=\widehat{\rho}\left(D(\alpha),\left(\begin{array}{ll}
D(-\alpha) & \\
& D(\alpha)
\end{array}\right)\right)=\left(\begin{array}{ll}
{ }^{1_{\mathrm{SO}(2)}} & \\
& D(2 \alpha)
\end{array}\right)=\zeta_{p}(D(2 \alpha))=\left(\zeta_{p} \circ \rho_{2}\right)(S(\alpha))
$$

and

$$
\left(\widehat{\rho} \circ \mathrm{sq} \circ \tilde{\zeta}_{l}\right)(S(\alpha))=\widehat{\rho}\left(1_{\mathrm{SO}(2)}, \tilde{D}(2 \alpha)\right)=\tilde{D}(2 \alpha)=\zeta_{l}(D(2 \alpha))=\left(\zeta_{l} \circ \rho_{2}\right)(S(\alpha)) .
$$

For the second claim observe that

$$
\begin{aligned}
&(\widehat{\rho})^{-1}\left(\zeta_{p}\right)(\operatorname{SO}(2))=\left\{\left(D(\alpha),\left({ }^{D(-\alpha)}{ }_{D(\alpha)}\right)\right) \mid \alpha \in \mathbb{R}\right\} \cong \operatorname{Spin}(2), \\
&(\widehat{\rho})^{-1}\left(\zeta_{l}\right)(\mathrm{SO}(2))=\{( \pm \mathrm{id}, \tilde{D}(\alpha)) \mid \alpha \in \mathbb{R}\} \cong\{1,-1\} \times \mathrm{SO}(2) .
\end{aligned}
$$

Consequence 14.11. One has

$$
\zeta_{p}=\varepsilon_{34} \quad \zeta_{l}=\varepsilon_{23} \cdot \varepsilon_{14}
$$

Proof. By Remarks 6.13 and 14.7 and Lemma 14.10 this is immediate from Lemma 14.9 ,

Proposition 14.12. Given $\tau \in \operatorname{Aut}\left(\mathrm{U}_{2}(\mathbb{C})\right)$, there is a unique $\tilde{\tau} \in \operatorname{Aut}\left(\mathrm{SO}(2) \times \mathrm{SU}_{2}(\mathbb{C})\right)$ such that

$$
\hat{\rho} \circ \tilde{\tau}=\tau \circ \widehat{\rho} .
$$

Proof. Let $\tau \in \operatorname{Aut}\left(\mathrm{U}_{2}(\mathbb{C})\right)$. Consider the characteristic subgroups

$$
H_{1}:=Z\left(\mathrm{U}_{2}(\mathbb{C})\right)=\left\{z \cdot I_{2} \mid z \in \mathrm{U}_{1}(\mathbb{C}) \cong \mathrm{SO}(2)\right\}, \quad H_{2}:=\left[\mathrm{U}_{2}(\mathbb{C}), \mathrm{U}_{2}(\mathbb{C})\right]=\mathrm{SU}_{2}(\mathbb{C}),
$$

of $\mathrm{U}_{2}(\mathbb{C})$. Note that $H_{1}$ and $H_{2}$ are also characteristic in $H:=H_{1} \times H_{2}$, as $H_{1}=Z(H)$ and $H_{2}=[H, H]$. Hence $\operatorname{Aut}(H)=\operatorname{Aut}\left(H_{1}\right) \times \operatorname{Aut}\left(H_{2}\right)$.

Let $\tau_{1}:=\tau_{\mid H_{1}} \in \operatorname{Aut}\left(H_{1}\right)$ and $\tau_{2}:=\tau_{\mid H_{2}} \in \operatorname{Aut}\left(H_{2}\right)$. Then $\tilde{\tau}:=\left(\tau_{1}, \tau_{2}\right) \in \operatorname{Aut}(H)$ satisfies

$$
(\widehat{\rho} \circ \tilde{\tau})(z, A)=\widehat{\rho}\left(\tau_{1}(z), \tau_{2}(A)\right)=\tau_{1}(z) \tau_{2}(A)=\tau(z) \tau(A)=\tau(z A)=(\tau \circ \widehat{\rho})(z, A)
$$

for all $(z, A) \in \mathrm{SO}(2) \times \mathrm{SU}_{2}(\mathbb{C})$. Let $\psi=\left(\psi_{1}, \psi_{2}\right) \in \operatorname{Aut}\left(\mathrm{SO}(2) \times \mathrm{SU}_{2}(\mathbb{C})\right)$ be such that $\hat{\rho} \circ \psi=\tau \circ \widehat{\rho}$. Given $z \in \mathrm{SO}(2)$ and $A \in \mathrm{SU}_{2}(\mathbb{C})$, one has

$$
\psi_{1}(z)=\psi_{1}(z) \psi_{2}\left(I_{2}\right)=(\hat{\rho} \circ \psi)\left(z, I_{2}\right)=(\tau \circ \widehat{\rho})\left(z, I_{2}\right)=(\widehat{\rho} \circ \tilde{\tau})\left(z, I_{2}\right)=\tau_{1}(z) \tau_{2}\left(I_{2}\right)=\tau_{1}(z)
$$

and

$$
\psi_{2}(A)=(\widehat{\rho} \circ \psi)\left(1_{\mathrm{SO}(2)}, A\right)=(\widehat{\rho} \circ \tilde{\tau})\left(1_{\mathrm{SO}(2)}, A\right)=\tau_{2}(A) .
$$

The final result of this section allows us to carry out Strategy 12.1 for edges of type $\mathrm{C}_{2}$ in Theorem 17.10 below. 
Proposition 14.13. There is an epimorphism $\tilde{\zeta}: \mathrm{SO}(2) \times \mathrm{SU}_{2}(\mathbb{C}) \rightarrow \operatorname{Spin}(3)$ such that

$$
\tilde{\zeta} \circ \tilde{\zeta}_{p}=\tilde{\varepsilon}_{12} \circ \operatorname{inv}, \quad \tilde{\zeta} \circ \tilde{\zeta}_{l}=\tilde{\varepsilon}_{23}
$$

Proof. The map

$$
\begin{aligned}
\psi: \operatorname{Spin}(3) & \rightarrow \mathrm{SU}_{2}(\mathbb{C}) \\
a+b e_{1} e_{2}+c e_{2} e_{3}+d e_{3} e_{1} & \mapsto R_{a+b i+c j+d k}=\left(\begin{array}{cccc}
a & b & c & d \\
-b & a & -d & c \\
-c & d & a & -b \\
-d & -c & b & a
\end{array}\right)
\end{aligned}
$$

is a group isomorphism by Remarks 6.5(b) and 13.13(b) (see also $\left.\mathrm{SBG}^{+} 95,11.26\right]$ ), with the convention that the matrix group $\mathrm{SU}_{2}(\mathbb{C})$ acts $\mathbb{R}$-linearly on $\mathbb{C}^{2} \cong \mathbb{H}$ with respect to the $\mathbb{R}$-basis $1, i=i 1, j, k=i j$ as in Definition 14.3 and Notation 14.4. Let

$$
\tilde{\zeta}: \mathrm{SO}(2) \times \mathrm{SU}_{2}(\mathbb{C}) \rightarrow \operatorname{Spin}(3):(z, A) \mapsto \psi^{-1}(A)
$$

For $\alpha \in \mathbb{R}$, one has

$$
\left(\tilde{\zeta} \circ \tilde{\zeta}_{p}\right)(S(\alpha))=\tilde{\zeta}\left(D(\alpha),\left({ }_{D(\alpha)}^{D(-\alpha)}\right)\right)=\cos (\alpha)-\sin (\alpha) e_{1} e_{2}=\left(\tilde{\varepsilon}_{12} \circ \operatorname{inv}\right)(S(\alpha))
$$

and

$$
\left(\tilde{\zeta} \circ \tilde{\zeta}_{l}\right)(S(\alpha))=\tilde{\zeta}\left(1_{\mathrm{SO}(2)}, \tilde{D}(\alpha)\right)=\zeta(\tilde{D}(\alpha))=\cos (\alpha)+\sin (\alpha) e_{2} e_{3}=\tilde{\varepsilon}_{23}(S(\alpha))
$$

Corollary 14.14. (a) There exist epimorphisms $\zeta_{1}, \zeta_{2}, \zeta_{3}: \mathrm{U}_{2}(\mathbb{C}) \rightarrow \mathrm{SO}(3)$ such that

$$
\begin{aligned}
\zeta_{1} \circ \zeta_{p}=\varepsilon_{12} \circ \text { inv }, & \zeta_{1} \circ \zeta_{l}=\varepsilon_{23}, \\
\zeta_{2} \circ \zeta_{p}=\varepsilon_{12}, & \zeta_{2} \circ \zeta_{l}=\varepsilon_{23}, \\
\zeta_{3} \circ \zeta_{p}=\varepsilon_{23}, & \zeta_{3} \circ \zeta_{l}=\varepsilon_{12} .
\end{aligned}
$$

(b) There exist epimorphisms $\tilde{\zeta}_{2}, \tilde{\zeta}_{3}: \mathrm{SO}(2) \times \mathrm{SU}_{2}(\mathbb{C}) \rightarrow \operatorname{Spin}(3)$ such that

$$
\begin{array}{ll}
\tilde{\zeta}_{2} \circ \tilde{\zeta}_{p}=\tilde{\varepsilon}_{12}, & \tilde{\zeta}_{2} \circ \tilde{\zeta}_{l}=\tilde{\varepsilon}_{23}, \\
\tilde{\zeta}_{3} \circ \tilde{\zeta}_{p}=\tilde{\varepsilon}_{23}, & \tilde{\zeta}_{3} \circ \tilde{\zeta}_{l}=\tilde{\varepsilon}_{12} .
\end{array}
$$

Proof. The kernel of the epimorphism $\mathrm{SO}(2) \times \mathrm{SU}_{2}(\mathbb{C}) \rightarrow \mathrm{U}_{2}(\mathbb{C}):(z, A) \mapsto z A$ is equal to $\langle(D(\pi), \tilde{D}(\pi))\rangle$. One has $\tilde{\zeta}(D(\pi), \tilde{D}(\pi))=\psi^{-1}\left(-I_{4}\right)=-1_{\operatorname{Spin}(3)}$, whence $\langle(D(\pi), \tilde{D}(\pi))\rangle \subseteq \operatorname{ker}\left(\rho_{3} \circ \tilde{\zeta}\right)$. Therefore, the claim concerning $\zeta_{1}$ follows from Proposition 14.13 and the homomorphism theorem of groups.

The claims about $\zeta_{2}, \zeta_{3}$ then follow from Lemma 9.7, resp. 9.4, A subsequent application of Proposition 8.4 yields the claims about $\tilde{\zeta}_{2}, \tilde{\zeta}_{3}$.

\section{NON-SPHERICAL DIAGRAMS OF RANK TWO}

In this section we prepare Strategy 12.1 for non-spherical diagrams of rank two. For an introduction to the concept of a Kac-Moody root datum we refer the reader to [Tit87, Introduction], Rém02, 7.1.1, p. 172], Mar13, Definition 5.1]. For the definition of simple connectedness see Rém02, 7.1.2]. Note that for a given generalized Cartan matrix, up to isomorphism, there exists a uniquely determined simply connected Kac-Moody root datum.

Definition 15.1. Let $r, s \in \mathbb{N}$ such that $r s \geq 4$ and consider the generalized Cartan matrix of rank two given by

$$
A:=(a(i, j))_{i \in\{1,2\}}=\left(\begin{array}{cc}
2 & -r \\
-s & 2
\end{array}\right)
$$

and a simply connected Kac-Moody root datum $\mathcal{D}=\left(\{1,2\}, A, \Lambda,\left(c_{i}\right)_{i \in\{1,2\}},\left(h_{i}\right)_{i \in\{1,2\}}\right)$.

Let $G:=G(A):=G(\mathcal{D})$ be the corresponding (simply connected) real Kac-Moody group of rank two, let $T_{0}$ be the fundamental torus of $G$ with respect to the fundamental roots $\alpha_{1}, \alpha_{2}$, and let

$$
K:=K^{r, s}:=K(A)
$$

be the subgroup consisting of the elements fixed by the Cartan-Chevalley involution with respect to $T_{0}$ of $G$ (its maximal compact subgroup). 
Remark 15.2. Let $G_{i} \cong \mathrm{SL}_{2}(\mathbb{R})$ be the corresponding fundamental subgroups of rank one and define

$$
K_{i}:=K_{i}^{r, s}:=G_{i} \cap K^{r, s} \cong \mathrm{SO}(2) \quad \text { and } \quad T:=T_{0} \cap K .
$$

By (KMG3) (see [Tit87, p. 545] or, e.g., Mar13, p. 84]), the torus $T_{0}$ is generated by $\mu^{h_{i}}$ for $i=1,2$ and $\mu \in \mathbb{R} \backslash\{0\}$ arbitrary. The action of the Cartan-Chevalley involution on the torus is given by $\mu^{h_{i}} \mapsto\left(\mu^{-1}\right)^{h_{i}}=\mu^{-h_{i}}$. Hence

$$
T=T_{0} \cap K=\left\{\mathrm{id}=1^{h_{1}}=1^{h_{2}}, t_{1}:=(-1)^{h_{1}}, t_{2}:=(-1)^{h_{2}}, t_{1} t_{2}=(-1)^{h_{1}+h_{2}}\right\} \cong \mathbb{Z} / 2 \mathbb{Z} \times \mathbb{Z} / 2 \mathbb{Z} .
$$

Lemma 15.3. $K$ is isomorphic to a free amalgamated product

$$
K_{1} T *_{T} K_{2} T
$$

Proof. The twin building of the Kac-Moody group $G$ is a twin tree (cf. [RT87, [RT94]). The chambers are the edges; the panels are the sets of edges sharing one vertex and, hence, correspond to vertices. The group $K$ acts edge-transitively and without inversions on each half of the twin tree of $G$ by the Iwasawa decomposition (see, e.g., DMGH09]). The Cartan-Chevalley involution $\omega$ interchanges the two halves of the twin tree, mapping edges to opposite edges. Hence the stabilizer in $K$ of the fundamental edge $c^{+}$ also stabilizes the opposite edge $c^{-}=\omega\left(c^{+}\right)$and, thus, the unique twin apartment spanned by them. It follows that the edge stabilizer is $T$. Since the panels correspond to the vertices of the tree, the stabilizers of the vertices of the fundamental edge $c^{+}$are equal to $K_{1} T$ and $K_{2} T$. The claim follows from [Ser03, I, $\S 5]$.

Remark 15.4. Since $K_{i} \unlhd K_{i} T$, this free amalgamated product is fully determined by the intersections $K_{i} \cap T$ and the action of $T$ on each $K_{i}$. Note that (KMG3) implies $T \cap K_{1}=\left\{1, t_{1}\right\}$ and $T \cap K_{2}=\left\{1, t_{2}\right\}$. The action of $T$ on each $K_{i}$ can be extracted from the action of $T_{0}$ on each $G_{i}$, which according to Tit87, (4), p. 549] (or also Mar13, (5.1), p. 86]) is given by

$$
t x_{i}(\lambda) t^{-1}=x_{i}\left(t\left(c_{i}\right) \lambda\right)
$$

for $t \in T_{0}, \lambda \in \mathbb{R}$ and root group functor $x_{i}$. According to [Tit87, Section 2, p. 544] (or also [Mar13, Definitions 5.1 and 5.5]) one computes for $i, j \in\{1,2\}$ that

$$
t_{i}\left(c_{j}\right)=(-1)^{h_{i}}\left(c_{j}\right)=(-1)^{h_{i}\left(c_{j}\right)}=(-1)^{a(i, j)} .
$$

We conclude that $t_{i}$ acts trivially on $K_{j}$, if and only if the entry $a(i, j)$ of the generalized Cartan matrix is even; conversely it acts non-trivially (and hence by inversion) if and only if $a(i, j)$ is odd.

In symbols, for

and $k_{j} \in K_{j}$ one has

$$
n(i, j):= \begin{cases}0, & \text { if } a(i, j) \text { is even } \\ 1, & \text { if } a(i, j) \text { is odd }\end{cases}
$$

$$
t_{i}^{-1} k_{j} t_{i}=k_{j}^{-2 n(i, j)} k_{j}
$$

Notation 15.5. Let

$$
\theta_{1}:=\theta_{1}^{r, s}: \mathrm{SO}(2) \rightarrow K_{1}^{r, s}, \quad \theta_{2}:=\theta_{2}^{r, s}: \mathrm{SO}(2) \rightarrow K_{2}^{r, s},
$$

be continuous isomorphisms.

Definition 15.6. Let $r, s \in \mathbb{N}$ such that $r s \geq 4$, let $A=\left(\begin{array}{cc}2 & -r \\ -s & 2\end{array}\right)$. Then set

$$
H^{r, s}:=\left\{\begin{array}{lll}
\mathrm{SO}(2) \times \mathrm{SO}(2) & \text { if } r \equiv s \equiv 0 & (\bmod 2) \\
\mathrm{SO}(3) & \text { if } r \equiv s \equiv 1 \quad(\bmod 2) \\
\mathrm{U}_{2}(\mathbb{C}) & \text { otherwise. }
\end{array}\right.
$$

Also set

$$
\delta_{1}:=\left\{\begin{array}{lll}
\iota_{1} & \text { if } r \equiv s \equiv 0 \quad(\bmod 2), \\
\varepsilon_{12} & \text { if } r \equiv s \equiv 1 \quad(\bmod 2), \\
\zeta_{l} & \text { if } r \equiv 0, s \equiv 1 \quad(\bmod 2), \\
\zeta_{p} & \text { if } r \equiv 1, s \equiv 0 \quad(\bmod 2),
\end{array} \delta_{2}:=\left\{\begin{array}{llll}
\iota_{2} & \text { if } r \equiv s \equiv 0 \quad(\bmod 2) & (\text { see } 8.1) \\
\varepsilon_{23} & \text { if } r \equiv s \equiv 1 \quad(\bmod 2) & (\text { see 5.2) } \\
\zeta_{p} & \text { if } r \equiv 0, s \equiv 1 \quad(\bmod 2) & (\text { see } 14.3) \\
\zeta_{l} & \text { if } r \equiv 1, s \equiv 0 & (\bmod 2)
\end{array}\right.\right.
$$

Proposition 15.7. Let $r, s \in \mathbb{N}$ such that $r s \geq 4$, let $A=\left(\begin{array}{cc}2 & -r \\ -s & 2\end{array}\right)$, let $G(A)$ be the corresponding simply connected real Kac-Moody group, and let $K^{r, s}$ be its maximal compact subgroup. Then there exists a group epimorphism $\theta: K^{r, s} \rightarrow H^{r, s}$ such that

$$
\theta \circ \theta_{1}=\delta_{1} \quad \text { and } \quad \theta \circ \theta_{2}=\delta_{2} .
$$


Proof. By Lemma 15.3 the group $K$ is isomorphic to $K_{1} T *_{T} K_{2} T$ with $T=\left\{1, t_{1}, t_{2}, t_{1} t_{2}\right\} \cong \mathbb{Z} / 2 \mathbb{Z} \times \mathbb{Z} / 2 \mathbb{Z}$ and $T_{0} \cap K_{1}=\left\{1, t_{1}\right\}, T_{0} \cap K_{2}=\left\{1, t_{2}\right\}$; in particular, $K$ is generated by $K_{1} \cong \mathrm{SO}(2)$ and $K_{2} \cong \mathrm{SO}(2)$. As $K$ is a free amalgamated product it therefore suffices to define $\theta$ on each of the $K_{i}$ and to verify that the actions of the $t_{i}$ on the $K_{j}$ are compatible with the actions of the images of the $t_{i}$ on the images of the $K_{j}$. Define $\theta$ via

$$
\theta_{\mid K_{1}}: K_{1} \rightarrow \delta_{1}(\mathrm{SO}(2)): x \mapsto\left(\delta_{1} \circ \theta_{1}^{-1}\right)(x) \quad \text { and } \quad \theta_{\mid K_{2}}: K_{2} \rightarrow \delta_{2}(\operatorname{SO}(2)): x \mapsto\left(\delta_{2} \circ \theta_{2}^{-1}\right)(x) .
$$

Then this is compatible with the action of $T$. Indeed, using Remark 15.4, one observes:

(a) Since $r \equiv s \equiv 0(\bmod 2)$, the elements $t_{i}$ centralize the groups $K_{j}$ which is compatible with the fact that $\mathrm{SO}(2) \times \mathrm{SO}(2)$ is an abelian group.

(b) Since $r \equiv s \equiv 1(\bmod 2)$, the element $t_{1}$ inverts the group $K_{2}$ and the element $t_{2}$ inverts the group $K_{1}$ which is compatible with the situation in $\mathrm{SO}(3)$ by Lemma 9.7 .

(c) Since $r \equiv 0(\bmod 2), s \equiv 1(\bmod 2)$, the element $t_{1}$ centralizes $K_{2}$ and the element $t_{2}$ inverts the group $K_{1}$. This is compatible with the following computations (cf. Definition 14.3):

$$
\begin{aligned}
& \forall g \in K_{2}: \theta\left(t_{1} g t_{1}\right)=\left(\begin{array}{cccc}
-1 & & 0 & \\
& -1 & & 0 \\
0 & 0 & -1 & \\
& 0 & & -1
\end{array}\right)\left(\begin{array}{cccc}
1 & & & \\
& 1 & & y \\
& x & y \\
& -y & x
\end{array}\right)\left(\begin{array}{cccc}
-1 & & 0 & \\
0 & -1 & & 0 \\
& 0 & & -1
\end{array}\right)=\left(\begin{array}{cccc}
1 & & & \\
& 1 & x & y \\
& & -y & x
\end{array}\right)=\theta(g),
\end{aligned}
$$

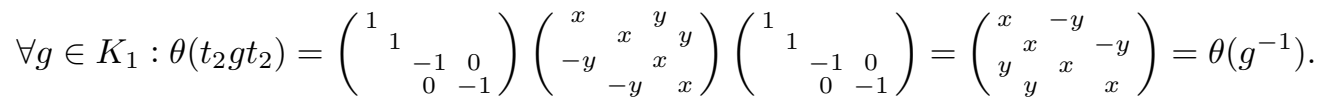

(d) This is dual to (c).

Definition 15.8. Let $r, s \in \mathbb{N}$ such that $r s \geq 4$, let $A=\left(\begin{array}{cc}2 & -r \\ -s & 2\end{array}\right)$. Then set

$$
\widetilde{H}^{r, s}:=\left\{\begin{array}{lll}
\operatorname{Spin}(2) \times \operatorname{Spin}(2) & \text { if } r \equiv s \equiv 0 & (\bmod 2), \\
\operatorname{Spin}(3) & \text { if } r \equiv s \equiv 1 \quad(\bmod 2), \\
\operatorname{SO}(2) \times \mathrm{SU}_{2}(\mathbb{C}) & \text { otherwise }
\end{array}\right.
$$

Furthermore, set

$$
\tilde{\delta}_{1}:=\left\{\begin{array}{ll}
\tilde{\iota}_{1} & \text { if } r \equiv s \equiv 0 \quad(\bmod 2), \\
\tilde{\varepsilon}_{12} & \text { if } r \equiv s \equiv 1 \quad(\bmod 2), \\
\tilde{\zeta}_{l} & \text { if } r \equiv 0, s \equiv 1 \quad(\bmod 2), \\
\tilde{\zeta}_{p} & \text { if } r \equiv 1, s \equiv 0 \quad(\bmod 2),
\end{array} \quad \tilde{\delta}_{2}:=\left\{\begin{array}{llll}
\tilde{\iota}_{2} & \text { if } r \equiv s \equiv 0 \quad(\bmod 2) & (\text { see } 8.1) \\
\tilde{\varepsilon}_{23} & \text { if } r \equiv s \equiv 1 \quad(\bmod 2) & (\text { see 6.10) } \\
\tilde{\zeta}_{p} & \text { if } r \equiv 0, s \equiv 1 \quad(\bmod 2) & (\text { see 14.8) } \\
\tilde{\zeta}_{l} & \text { if } r \equiv 1, s \equiv 0 & (\bmod 2)
\end{array}\right.\right.
$$

The central extension

$$
\bar{\rho}: \widetilde{H}^{r, s} \rightarrow H^{r, s}
$$

satisfies

$$
\bar{\rho}=\left\{\begin{array}{llll}
\rho_{2} \times \rho_{2} & \text { if } r \equiv s \equiv 0 & (\bmod 2) & (\text { see } 8.1) \\
\rho_{3} & \text { if } r \equiv s \equiv 1 & (\bmod 2) & (\text { see 6.8) } \\
\widehat{\rho} & \text { otherwise } & & (\text { see 14.8) }
\end{array}\right.
$$

Let $K^{r, s}=K(A)=K_{1} T *_{T} K_{2} T$ as in Lemma 15.3 and let $t_{1} \in K_{1} \cap T, t_{2} \in K_{2} \cap T$ as in Remark 15.4,

Define

$$
\begin{aligned}
& u_{i}:=\theta\left(t_{i}\right) \quad(\text { see 15.7), } \\
& U:=\left\langle u_{1}, u_{2}\right\rangle \cong \mathbb{Z} / 2 \mathbb{Z} \times \mathbb{Z} / 2 \mathbb{Z} .
\end{aligned}
$$

Furthermore, define

$$
\begin{aligned}
& \widetilde{U}:=\bar{\rho}^{-1}(U), \\
& \widetilde{K}_{i}:=\widetilde{K}_{i}^{r, s}:=\bar{\rho}^{-1}\left(\theta\left(K_{i}\right)\right),
\end{aligned}
$$

and the spin extension

$$
\widetilde{K}:=\widetilde{K}^{r, s}:=\widetilde{K}(A):=\widetilde{K}_{1} \widetilde{U} *_{\widetilde{U}} \widetilde{K}_{2} \widetilde{U} \quad \text { of } \quad K=K^{r, s}=K(A),
$$

let

$$
\hat{\hat{\rho}}: \widetilde{K}_{1} \widetilde{U} *_{\widetilde{U}} \widetilde{K}_{2} \widetilde{U} \rightarrow K_{1} T *_{T} K_{2} T
$$

be the epimorphism induced by $\bar{\rho}_{\mid \widetilde{K}_{1}}, \bar{\rho}_{\mid \widetilde{K}_{2}}$ and let

$$
\tilde{\theta}_{1}: \operatorname{Spin}(2) \rightarrow \widetilde{K}_{1} \quad \text { and } \quad \tilde{\theta}_{2}: \operatorname{Spin}(2) \rightarrow \widetilde{K}_{2}
$$


be continuous monomorphisms such that the following diagrams commute for $i=1,2$ :

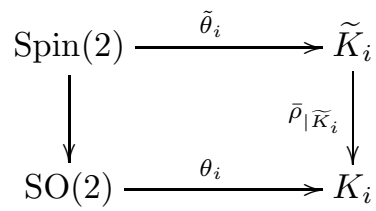

Remark 15.9. One has $\widetilde{K}_{1} \cong \operatorname{Spin}(2)$ unless $r \equiv 0, s \equiv 1(\bmod 2)$ and $\widetilde{K}_{2} \cong \operatorname{Spin}(2)$ unless $r \equiv 1, s \equiv 0$ $(\bmod 2)$, in which case the respective group is isomorphic to $\{1,-1\} \times \operatorname{SO}(2)$ (cf. Lemma 14.10). Hence $\tilde{\theta}_{1}$ actually is a (continuous) isomorphism unless $r \equiv 0, s \equiv 1(\bmod 2)$, in which case it is a (continuous) isomorphism onto the unique connected subgroup of index two of $\widetilde{K}_{1}$.

For $i=1$ the map on the left hand side of the above commutative diagram is

$$
\begin{aligned}
& \operatorname{Spin}(2) \rightarrow \mathrm{SO}(2) \quad: \quad S(\alpha) \mapsto D(\alpha) \quad \text { if } r \equiv 0, s \equiv 1 \quad(\bmod 2) \quad \text { (see 8.1) } \\
& \operatorname{Spin}(2) \rightarrow \mathrm{SO}(2) \quad: \quad x \mapsto \rho_{2}(x) \quad \text { otherwise. }
\end{aligned}
$$

The dual statement holds for $\tilde{\theta}_{2}$.

In particular, for $i=2$ the map on the left hand side of the above commutative diagram is

$$
\begin{aligned}
& \operatorname{Spin}(2) \rightarrow \operatorname{SO}(2) \quad: \quad S(\alpha) \mapsto D(\alpha) \quad \text { if } r \equiv 1, s \equiv 0 \quad(\bmod 2) \quad \text { (see 8.1) } \\
& \operatorname{Spin}(2) \rightarrow \mathrm{SO}(2) \quad: \quad x \mapsto \rho_{2}(x) \quad \text { otherwise. }
\end{aligned}
$$

Define

$$
\begin{aligned}
& \tilde{t}_{1}:= \begin{cases}\tilde{\theta}_{1}(S(\pi)), & \text { if } r \equiv 0, s \equiv 1 \quad(\bmod 2) \\
\tilde{\theta}_{1}\left(S\left(\frac{\pi}{2}\right)\right), & \text { otherwise }\end{cases} \\
& \tilde{t}_{2}:= \begin{cases}\tilde{\theta}_{2}(S(\pi)), & \text { if } r \equiv 1, s \equiv 0 \quad(\bmod 2) \\
\tilde{\theta}_{2}\left(S\left(\frac{\pi}{2}\right)\right), & \text { otherwise }\end{cases}
\end{aligned}
$$

The following is true by construction:

Proposition 15.10. Let $r, s \in \mathbb{N}$ such that $r s \geq 4$, let $A=\left(\begin{array}{cc}2 & -r \\ -s & 2\end{array}\right)$, let $G(A)$ be the corresponding simply connected real Kac-Moody group, and let $K^{r, s}$ be its maximal compact subgroup, and let $\widetilde{K}^{r, s}$ be its spin extension. Then there exists a group epimorphism $\tilde{\theta}: \widetilde{K}^{r, s} \rightarrow \widetilde{H}^{r, s}$ such that

$$
\tilde{\theta} \circ \tilde{\theta}_{1}=\tilde{\delta}_{1} \quad \text { and } \quad \tilde{\theta} \circ \tilde{\theta}_{2}=\tilde{\delta}_{2} .
$$

Moreover, the following diagram commutes:

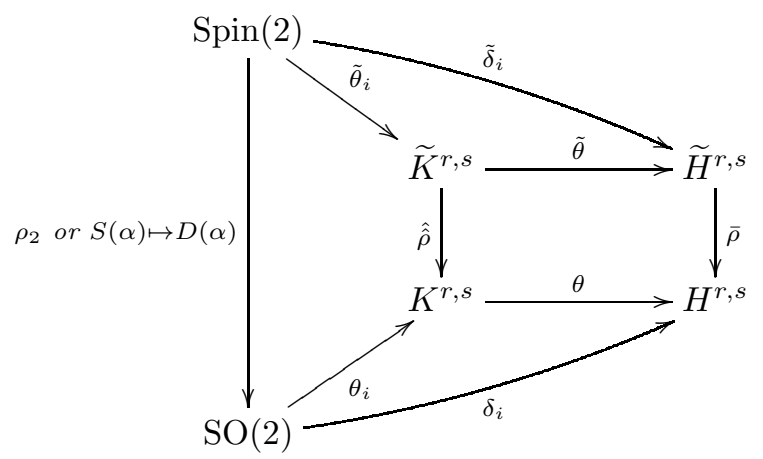

where the epimorphism on the left hand side is one of $\rho_{2}$ or $S(\alpha) \mapsto D(\alpha)$ as described in Remark 15.9.

Furthermore, for $\{i, j\}=\{1,2\}$ and $\tilde{k}_{j} \in \widetilde{K}_{j}$ one has

$$
\tilde{t}_{i}^{-1} \tilde{k}_{j} \tilde{t}_{i}=\tilde{k}_{j}^{-2 n(i, j)} \tilde{k}_{j} .
$$

Identity (2) follows from identity (1) in Remark 15.4

Remark 15.11. The Cartan matrix of type $\mathrm{C}_{2}$ over $\{1,2\}$ with short root $\alpha_{1}$ and long root $\alpha_{2}$ (i.e., $2 \rightarrow 1$; see Remark 4.3) is

$$
\left(\begin{array}{cc}
2 & -2 \\
-1 & 2
\end{array}\right)
$$


cf. [Car89, p. 44]. The group $H^{r, s}$ from Proposition 15.10 is of type $\mathrm{C}_{2}$ with short root $\alpha_{1}$ and long root $\alpha_{2}$ if and only if $r$ is even and $s$ is odd. We conclude that for $i=1$ the map on the left hand side of the commutative diagram is

$$
\operatorname{Spin}(2) \rightarrow \mathrm{SO}(2): S(\alpha) \mapsto D(\alpha)
$$

which is in accordance with Notation 14.8 and Lemma 14.10. In other words, in this example the rank one group corresponding to the long root is doubly covered by its spin cover and the rank one group corresponding to the short root is singly covered by its spin cover.

The direction introduced for edges labelled $\infty$ in Notation 4.4 was chosen to fit this observation: the arrow points away from the doubly covered vertex of the diagram towards the singly covered vertex of the diagram.

We point out that Bou02, Plate III, p. 269] incorrectly gives the transpose of the above matrix as the Cartan matrix of type $\mathrm{C}_{2}$ with short root $\alpha_{1}$ and long root $\alpha_{2}$.

Remark 15.12. If both $r, s$ are odd, then in $\widetilde{K}=\widetilde{K}_{1} \widetilde{U} *_{\widetilde{U}} \widetilde{K}_{2} \widetilde{U}$ one has

$$
\widetilde{U} \cong Q_{8}
$$

In other words, the element $S(\pi)$ of the group $\widetilde{K}_{1} \cong \operatorname{Spin}(2)$ is identical to the element $S(\pi)$ of the group $\widetilde{K}_{2} \cong \operatorname{Spin}(2)$, like for the groups $\tilde{\varepsilon}_{12}(\operatorname{Spin}(2))$ and $\tilde{\varepsilon}_{23}(\operatorname{Spin}(2))$ in $\operatorname{Spin}(3)$. The same is true for the groups $\tilde{\eta}_{p}(\operatorname{Spin}(2))$ and $\tilde{\eta}_{l}(\operatorname{Spin}(2))$ in $\operatorname{Spin}(4)$ by Proposition 13.15,

Remark 15.13. If both $r$ and $s$ are even, then in $\widetilde{K}=\widetilde{K}_{1} \widetilde{U} \widetilde{U}_{\widetilde{K}} \widetilde{K}_{2} \widetilde{U}$ one has

$$
\widetilde{U} \cong \mathbb{Z} / 4 \mathbb{Z} \times \mathbb{Z} / 4 \mathbb{Z}
$$

if one of $r, s$ is even and the other odd, then one has

$$
\widetilde{U} \cong \mathbb{Z} / 4 \mathbb{Z} \times \mathbb{Z} / 2 \mathbb{Z}
$$

Lemma 15.14. Each automorphism $\alpha$ of $K=K_{1} U *_{U} K_{2} U$ and each automorphism $\widetilde{\alpha}$ of $\widetilde{K}=\widetilde{K}_{1} \widetilde{U} *_{\widetilde{U}}$ $\widetilde{K}_{2} \widetilde{U}$ induces an automorphism of the Bruhat-Tits tree of $G$. The set $\left\{\alpha\left(K_{1} U\right), \alpha\left(K_{2} U\right)\right\}$ is $K$-conjugate to $\left\{K_{1} U, K_{2} U\right\}$, the set $\left\{\widetilde{\alpha}\left(\widetilde{K}_{1} \widetilde{U}\right), \widetilde{\alpha}\left(\widetilde{K}_{2} \widetilde{U}\right)\right\}$ is $\widetilde{K}$-conjugate to $\left\{\widetilde{K}_{1} \widetilde{U}, \widetilde{K}_{2} \widetilde{U}\right\}$.

Proof. $K_{1} U$ and $K_{2} U$ are indecomposable as amalgamated products and do not admit $\mathbb{Z}$ as a quotient. Moreover, $U$ is finite. Therefore by [KS70, Theorem 6], each $\alpha\left(K_{i} U\right)$ is conjugate to $K_{1} U$ or to $K_{2} U$. Hence $\alpha\left(K_{1} U\right), \alpha\left(K_{2} U\right)$ each stabilize a vertex of the Bruhat-Tits tree $X$ of $G$. Since $\alpha\left(K_{1} U\right) \cap \alpha\left(K_{2} U\right)=$ $\alpha(U)$, these two vertices are adjacent and $\alpha$ acts on $X$. Since $K$ acts edge-transitively on $X$ the set $\left\{\alpha\left(K_{1} U\right), \alpha\left(K_{2} U\right)\right\}$ is conjugate to $\left\{K_{1} U, K_{2} U\right\}$. The same argument works for $\widetilde{\alpha}$ and $\widetilde{K}_{i} \widetilde{U}$.

Proposition 15.15. For each automorphism $\alpha$ of $K$ there exists a unique automorphism $\widetilde{\alpha}$ of $\widetilde{K}$ such that

$$
\hat{\hat{\rho}} \circ \widetilde{\alpha}=\alpha \circ \hat{\hat{\rho}}
$$

Proof. Since $K$ modulo its centre is isomorphic to $\widetilde{K}$ modulo its centre, the claim holds for inner automorphisms. It therefore suffices to study the outer automorphisms groups $\operatorname{Out}(\cdot)=\operatorname{Aut}(\cdot) / \operatorname{Inn}(\cdot)$. By Lemma 15.14 it therefore suffices to investigate automorphisms that preserve the sets $K_{1} U \cup K_{2} U$, resp. $\widetilde{K}_{1} \widetilde{U} \cup \widetilde{K}_{2} \widetilde{U}$. The claim follows from Proposition 8.2

\section{Part IV. Arbitrary diagrams}

\section{Admissible COLOURings}

In this section we extend the classification results for $\mathrm{SO}(2)$ - and $\mathrm{Spin}(2)$-amalgams from Sections 9 and 10 to arbitrary diagrams.

Remark 16.1. Throughout this section, let $A=(a(i, j))_{i, j \in I}$ be a generalized Cartan matrix over the index set $I$ and let $\Pi$ be the augmented Dynkin diagram with vertex set $V$ induced by $A$ (see Notation 4.4) with respect to a labelling $\sigma: I \rightarrow V$.

We have seen in Proposition 15.10 that - given two vertices $i^{\sigma}, j^{\sigma}$ of $\Pi$ - some subtleties related to single versus double covers of the circle group arise in the theory of spin covers of rank two depending on the parities of $a(i, j)$ and $a(j, i)$. To this end we develop a theory of admissible colourings that will help us distinguish the respective vertices of the diagram from one another. 
Definition 16.2. For $i \neq j \in I$, define

$$
n(i, j):= \begin{cases}0, & \text { if } a(i, j) \text { is even } \\ 1, & \text { if } a(i, j) \text { is odd }\end{cases}
$$

Let $\Pi^{\text {adm }}$ be the graph on the vertex set $V$ with edge set

$$
\left\{\{i, j\}^{\sigma} \in V \times V \mid i \neq j \in I, n(i, j)=n(j, i)=1\right\} .
$$

An admissible colouring of $\Pi$ is a map $\kappa: V \rightarrow\{1,2\}$ such that

(a) $\kappa\left(i^{\sigma}\right)=1$ whenever there exists $j \in I \backslash\{i\}$ with $n(i, j)=0$ and $n(j, i)=1$,

(b) the restriction of $\kappa$ to any connected component of the graph $\Pi^{\text {adm }}$ is a constant map.

Let $c(\Pi, \kappa)$ be the number of connected components of $\Pi^{\text {adm }}$ on which $\kappa$ takes the value 2 .

An admissible colouring $\kappa$ is elementary if $c(\Pi, \kappa)=1$, i.e., if there exists a unique connected component of the graph $\Pi^{\text {adm }}$ on which $\kappa$ takes the value 2 . The admissible colouring $\kappa \equiv 1$ (i.e., the one with $c(\Pi, \kappa)=0)$ is called the trivial colouring. An admissible colouring $\kappa$ is proper if every connected component of $\Pi$ contains a vertex $v$ with $\kappa(v)=2$.

Remark 16.3. An elementary admissible colouring is given by assigning the value 2 to exactly one connected component of $\Pi^{\text {adm }}$, while all other connected components take value 1 . Therefore, in order to construct all elementary admissible colourings, it suffices to decide which connected components may be assigned the value 2 .

Let $k^{\sigma}$ be a vertex of $\Pi$. The only obstruction to being able to assign the value 2 to $k^{\sigma}$ is being contained in the connected component of $\Pi^{\text {adm }}$ of a vertex that necessarily has to be assigned value 1 . That is, there exists a vertex $i^{\sigma}$ in the same connected component of $\Pi^{\text {adm }}$ as $k^{\sigma}$ such that there is a vertex $j^{\sigma}$ with $n(i, j)=0$ and $n(j, i)=1$.

Lemma 16.4. Consider the partial order $\preceq$ on the set of admissible colouring of $\Pi$ where $\kappa \preceq \kappa^{\prime}$ if $\kappa(v) \leq \kappa^{\prime}(v)$ for all $v \in V$. Then there is a unique maximal admissible colouring $\kappa_{\max }$ of $\Pi$ with respect to $\preceq$.

Proof. Suppose $X$ is a set of admissible colourings of $\Pi$. Then one readily checks that $\kappa: V \rightarrow\{1,2\}$ : $v \mapsto \max \left\{\kappa^{\prime}(v) \mid \kappa^{\prime} \in X\right\}$ is again an admissible colouring, satisfying $\kappa^{\prime} \preceq \kappa$ for all $\kappa^{\prime} \in X$. Since $V$ is finite, so is its set of admissible colourings, i.e., there exists a maximal element.

Remark 16.5. Given two admissible colourings $\kappa_{1}, \kappa_{2}$ of $\Pi$, define the sum of $\kappa_{1}$ and $\kappa_{2}$ as

$$
\kappa_{1}+\kappa_{2}: V \rightarrow\{1,2\}: v \mapsto \max \left\{\kappa_{i}(v) \mid i=1,2\right\} .
$$

By the preceding discussion, this is again an admissible colouring. Each non-trivial admissible colouring of a Dynkin diagram is the sum of (finitely many pairwise distinct) elementary colourings. The maximal admissible colouring $\kappa_{\max }$ is the sum of all elementary colourings.

Notation 16.6. Throughout this section, let $\kappa: V \rightarrow\{1,2\}$ be an admissible colouring of $\Pi$. For $i \neq j \in I$, set

$$
v_{i j}:=v: \Leftrightarrow\{i, j\}^{\sigma} \in E_{v}(\Pi), \quad \kappa_{i j}:=\frac{\kappa\left(i^{\sigma}\right)+\kappa\left(j^{\sigma}\right)}{2} \in\{1,1.5,2\} .
$$

Note that $v_{i j}$ is well-defined since the sets $E_{v}(\Pi)$ for $v \in\{0,1,2,3, \infty\}$ form a partition of $\left(\begin{array}{c}V \\ 2\end{array}\right)$. Moreover, note that $\kappa_{i j}=1.5$ implies $v_{i j} \in\{0,2, \infty\}$ by Definition 16.2(b). Furthermore, $\kappa_{i j}=2$ implies $v_{i j} \in$ $\{0,1,3, \infty\}$ by Definition 16.2(a).

Remark 16.7. The labelling $\sigma$ and the colouring $\kappa$ allow one to extend the direction relation on the augmented Dynkin diagram $\Pi$ to a direction relation between all pairs of vertices $u \neq v \in V$ (cf. Remark 15.11):

- If $\{u, v\}$ is a directed edge, retain the direction $u \rightarrow v$.

- If $\kappa(u) \neq \kappa(v)$, then set $u \rightarrow v$ whenever $\kappa(u)>\kappa(v)$.

- For the remaining cases, use the labelling to introduce a direction as follows:

$$
\forall\{i, j\}^{\sigma} \text { non-directed with } \kappa\left(i^{\sigma}\right)=\kappa\left(j^{\sigma}\right): \quad i^{\sigma} \rightarrow j^{\sigma}: \Leftrightarrow i>j .
$$

This direction relation is called the orientation of $\Pi$ induced by the labelling $\sigma$ and the colouring $\kappa$.

In the following we will quite freely use the notation introduced in the sections in which we studied the rank two situation, such as Sections 6, 8, 13, 14, 15. 
Notation 16.8. For $i \neq j \in I$, let $r:=a(i, j)$ and $s:=a(j, i)$. Define

$$
G^{r, s}:= \begin{cases}\mathrm{SO}(2) \times \mathrm{SO}(2), & \text { if } r s=0, \\ \mathrm{SO}(3), & \text { if } r s=1, \\ \mathrm{U}_{2}(\mathbb{C}), & \text { if } r s=2, \\ \mathrm{SO}(4), & \text { if } r s=3, \\ K^{r, s}=K_{1} T *_{T} K_{2} T & \text { if } r s \geq 4,\end{cases}
$$

where in the final case - as discussed in Remark 15.4 - the action of $T$ on $K_{i}$ depends on the parities of the entries of the generalized Cartan matrix. Furthermore, define homomorphisms from $\mathrm{SO}(2)$ into $G^{r, s}$ as follows:

$$
\varepsilon_{1}^{r, s}:=\left\{\begin{array}{ll}
\iota_{1}, & \text { if } r s=0, \\
\varepsilon_{12}, & \text { if } r s=1, \\
\zeta_{p}, & \text { if } r s=2, \\
\eta_{p}, & \text { if } r s=3, \\
\theta_{1}^{r, s}, & \text { if } r s \geq 4,
\end{array} \quad \text { and } \quad \varepsilon_{2}^{r, s}:= \begin{cases}\iota_{2}, & \text { if } r s=0, \\
\varepsilon_{23}, & \text { if } r s=1, \\
\zeta_{l}, & \text { if } r s=2, \\
\eta_{l}, & \text { if } r s=3, \\
\theta_{2}^{r, s}, & \text { if } r s \geq 4 .\end{cases}\right.
$$

Next, define various covering groups of these:

$$
\widetilde{G}^{r, s, k}:= \begin{cases}G^{r, s}, & \text { if } k=1, \\ \operatorname{Spin}(2) \times \operatorname{Spin}(2), & \text { if } r s=0 \text { and } k>1, \\ \operatorname{Spin}(3), & \text { if } r s=1 \text { and } k=2, \\ \operatorname{SO}(2) \times \mathrm{SU}_{2}(\mathbb{C}), & \text { if } r s=2 \text { and } k=1.5, \\ \operatorname{Spin}(4), & \text { if } r s=3 \text { and } k=2, \\ \widetilde{K}^{r, s}=\widetilde{K}_{1} \widetilde{U} \widetilde{U}_{1} \widetilde{U}_{1} & \text { if } r s \geq 4 \text { and } k>1 .\end{cases}
$$

Recall from Notation 8.1 the isomorphism

$$
\psi: \mathrm{SO}(2) \rightarrow \operatorname{Spin}(2): D(\alpha) \mapsto S(\alpha) .
$$

Using this, define the following homomorphisms from $\operatorname{Spin}(2)$ into $\widetilde{G}^{(r, s, k)}$ :

$$
\tilde{\varepsilon}_{1}^{(r, s, k)}:=\left\{\begin{array}{ll}
\varepsilon_{1}^{r, s} \circ \psi^{-1} & \text { if } k=1 \\
\tilde{\iota}_{1}, & \text { if } r s=0 \text { and } k>1, \\
\tilde{\varepsilon}_{12}, & \text { if } r s=1 \text { and } k=2, \\
\tilde{\zeta}_{p}, & \text { if } r s=2 \text { and } k=1.5, \\
\tilde{\eta}_{p}, & \text { if } r s=3 \text { and } k=2, \\
\tilde{\theta}_{1}^{r, s}, & \text { if } r s \geq 4 \text { and } k>1,
\end{array} \quad \text { and } \quad \tilde{\varepsilon}_{2}^{r, s, k}:= \begin{cases}\varepsilon_{2}^{r, s} \circ \psi^{-1} & \text { if } k=1 \\
\tilde{\iota}_{2}, & \text { if } r s=0 \text { and } k>1, \\
\tilde{\varepsilon}_{23}, & \text { if } r s=1 \text { and } k=2, \\
\tilde{\zeta}_{l}, & \text { if } r s=2 \text { and } k=1.5, \\
\tilde{\eta}_{l}, & \text { if } r s=3 \text { and } k=2, \\
\tilde{\theta}_{2}^{r, s}, & \text { if } r s \geq 4 \text { and } k>1 .\end{cases}\right.
$$

Finally, define epimorphisms

$$
\rho^{r, s, k}: \widetilde{G}^{r, s, k} \rightarrow G^{r, s}
$$

via

$$
\rho^{r, s, 1}:=\mathrm{id}, \quad \rho^{r, s, 1.5}:=\left\{\begin{array}{ll}
\rho_{2} \times \psi^{-1}, & \text { if } r s=0 \text { and } i^{\sigma} \rightarrow j^{\sigma}, \\
\psi^{-1} \times \rho_{2}, & \text { if } r s=0 \text { and } i^{\sigma} \leftarrow j^{\sigma}, \\
\widehat{\rho}, & \text { if } r s=2, \\
\hat{\hat{\rho}}, & \text { if } r s \geq 4,
\end{array} \quad \rho^{r, s, 2} \quad:= \begin{cases}\rho_{2} \times \rho_{2}, & \text { if } r s=0, \\
\rho_{3}, & \text { if } r s=1, \\
\rho_{4}, & \text { if } r s=3, \\
\hat{\hat{\rho}}, & \text { if } r s \geq 4 .\end{cases}\right.
$$

Definition 16.9. (a) An $\mathrm{SO}(2)$-amalgam with respect to $\Pi$ and $\sigma$ is an amalgam

$$
\mathcal{A}=\left\{G_{i j}, \phi_{i j}^{i}, \mid i \neq j \in I\right\}
$$

such that for all $i \neq j \in I$, we have

$$
G_{i j}=G^{a(i, j), a(j, i)} \quad \text { and } \quad \phi_{i j}^{i}(\mathrm{SO}(2))= \begin{cases}\varepsilon_{1}^{a(i, j), a(j, i)}(\mathrm{SO}(2)), & \text { if } i^{\sigma} \rightarrow j^{\sigma}, \\ \varepsilon_{2}^{a(i, j), a(j, i)}(\mathrm{SO}(2)), & \text { if } i^{\sigma} \leftarrow j^{\sigma} .\end{cases}
$$

(b) The standard SO(2)-amalgam with respect to $\Pi$ and $\boldsymbol{\sigma}$ is the (continuous) $\mathrm{SO}(2)$-amalgam

$$
\mathcal{A}(\Pi, \sigma, \operatorname{SO}(2)):=\underset{42}{\left\{G_{i j}, \phi_{i j}^{i}, \mid i \neq j \in I\right\}}
$$


with respect to $\Pi$ and $\sigma$ such that for all $i \neq j \in I$, we have

$$
G_{i j}=G^{a(i, j), a(j, i))} \quad \text { and } \quad \phi_{i j}^{i}= \begin{cases}\varepsilon_{1}^{a(i, j), a(j, i)}, & \text { if } i^{\sigma} \rightarrow j^{\sigma}, \\ \varepsilon_{2}^{a(i, j), a(j, i)}, & \text { if } i^{\sigma} \leftarrow j^{\sigma} .\end{cases}
$$

(c) A Spin(2)-amalgam with respect to $\Pi, \sigma$ and $\kappa$ is an amalgam

$$
\mathcal{A}=\left\{G_{i j}, \phi_{i j}^{i}, \mid i \neq j \in I\right\}
$$

such that for all $i \neq j \in I$, we have

$$
G_{i j}=\widetilde{G}^{a(i, j), a(j, i), \kappa_{i j}} \quad \text { and } \quad \phi_{i j}^{i}(\operatorname{Spin}(2))= \begin{cases}\tilde{\varepsilon}_{1}^{a(i, j), a(j, i), \kappa_{i j}}(\operatorname{Spin}(2)), & \text { if } i^{\sigma} \rightarrow j^{\sigma}, \\ \tilde{\varepsilon}_{2}^{a(i, j), a(j, i), \kappa_{i j}}(\operatorname{Spin}(2)), & \text { if } i^{\sigma} \leftarrow j^{\sigma} .\end{cases}
$$

(d) The standard $\operatorname{Spin}(2)$-amalgam with respect to $\Pi, \sigma$ and $\kappa$ is the (continuous) $\operatorname{Spin}(2)$ amalgam

$$
\mathcal{A}(\Pi, \sigma, \kappa, \operatorname{Spin}(2)):=\left\{G_{i j}, \phi_{i j}^{i}, \mid i \neq j \in I\right\}
$$

with respect to $\Pi$ and $\sigma$ such that for all $i \neq j \in I$, we have

$$
G_{i j}=\widetilde{G}^{a(i, j), a(j, i), \kappa_{i j}} \quad \text { and } \quad \phi_{i j}^{i}= \begin{cases}\tilde{\varepsilon}_{1}^{a(i, j), a(j, i), \kappa_{i j}}, & \text { if } i^{\sigma} \rightarrow j^{\sigma}, \\ \tilde{\varepsilon}_{2}^{a(i, j), a(j, i), \kappa_{i j},}, & \text { if } i^{\sigma} \leftarrow j^{\sigma} .\end{cases}
$$

(e) Let $\mathcal{A}=\left\{G_{i j}, \phi_{i j}^{i} \mid i \neq j \in I\right\}$ be an $\mathrm{SO}(2)$-amalgam with respect to $\Pi$ and $\sigma$. Given $i \neq j \in I$, there is $\tau_{i j}^{i} \in \operatorname{Aut}(\mathrm{SO}(2))$ such that

$$
\phi_{i j}^{i}= \begin{cases}\varepsilon_{1}^{a(i, j), a(j, i), \kappa_{i j}} \circ \tau_{i j}^{i}, & \text { if } i^{\sigma} \rightarrow j^{\sigma}, \\ \varepsilon_{2}^{a(i, j), a(j, i), \kappa_{i j}} \circ \tau_{i j}^{i}, & \text { if } i^{\sigma} \leftarrow j^{\sigma} .\end{cases}
$$

Define $\tilde{\tau}_{i j}^{i} \in \operatorname{Aut}(\operatorname{Spin}(2))$ as in Lemma 8.2 and set

$$
\widetilde{G}_{i j}:=\widetilde{G}^{a(i, j), a(j, i), \kappa_{i j}} \quad \text { and } \quad \tilde{\phi}_{i j}^{i}:= \begin{cases}\tilde{\varepsilon}_{1}^{a(i, j), a(j, i), \kappa_{i j}} \circ \tilde{\tau}_{i j}^{i}, & \text { if } i^{\sigma} \rightarrow j^{\sigma}, \\ \tilde{\varepsilon}_{2}^{a(i, j), a(j, i), \kappa_{i j}} \circ \tilde{\tau}_{i j}^{i}, & \text { if } i^{\sigma} \leftarrow j^{\sigma} .\end{cases}
$$

Then $\widetilde{\mathcal{A}}:=\left\{\widetilde{G}_{i j}, \tilde{\phi}_{i j}^{i} \mid i \neq j \in I\right\}$ is the induced $\operatorname{Spin}(2)$-amalgam with respect to $\Pi, \sigma$ and $\kappa$.

Remark 16.10. In analogy to Remark10.5 the construction in Definition 16.9(e) is symmetric and can be applied backwards: Starting with a $\operatorname{Spin}(2)$-amalgam $\hat{\mathcal{A}}$, one can construct an $\mathrm{SO}(2)$-amalgam $\mathcal{A}$ such that $\hat{\mathcal{A}}=\widetilde{\mathcal{A}}$. As before, denote the corresponding epimorphism of amalgams by

$$
\pi_{\Pi, \sigma, \kappa}=\left\{\mathrm{id}, \rho^{i}, \rho_{i j}\right\}: \mathcal{A}(\Pi, \sigma, \kappa, \operatorname{Spin}(2)) \rightarrow \mathcal{A}(\Pi, \sigma, \mathrm{SO}(2)),
$$

where $\rho_{i j}=\rho^{a(i, j), a(j, i), \kappa_{i j}}$ and $\rho^{i}=\psi^{-1}$ for all $i \in I$ with $\kappa\left(i^{\sigma}\right)=1$ and $\rho^{i}=\rho_{2}$ for all $i \in I$ with $\kappa\left(i^{\sigma}\right)=2$.

Proposition 16.11. Let $\mathcal{A}_{1}$ and $\mathcal{A}_{2}$ be $\mathrm{SO}(2)$-amalgams with respect to $\Pi$ and $\sigma$, and let $\alpha=\left\{\pi, \alpha_{i j} \mid\right.$ $i \neq j \in I\}: \mathcal{A}_{1} \rightarrow \mathcal{A}_{2}$ be a colouring-preserving isomorphism of amalgams, i.e., for all $i \in I$ one has $\kappa\left(i^{\sigma}\right)=\kappa\left(i^{\pi \sigma}\right)$. Then there is a unique isomorphism $\tilde{\alpha}=\left\{\pi, \tilde{\alpha}_{i j} \mid i \neq j \in I\right\}: \widetilde{\mathcal{A}}_{1} \rightarrow \widetilde{\mathcal{A}}_{2}$ such that for all $i \neq j \in I$, one has

$$
\rho_{\pi(i) \pi(j)} \circ \tilde{\alpha}_{i j}=\alpha_{i j} \circ \rho_{i j} .
$$

Proof. This result generalizes Proposition 10.6. Its proof relies on the references

(a) Lemma 10.4.

(b) Proposition 8.4 resp. Corollary 8.3.

We indicate how one may adapt the proof of 10.6 in order to deal with the general situation. The cases $\left(k_{i j}, v_{i j}\right)=(2,0)$ and $\left(k_{i j}, v_{i j}\right)=(2,1)$ are covered by Proposition 10.6. For $\left(k_{i j}, v_{i j}\right)=(2,3)$ the proof is virtually identical to the proof for $\left(k_{i j}, v_{i j}\right)=(2,1)$, except that one uses $\rho_{4}$ instead of $\rho_{3}$, and Lemma 13.11 instead of Lemma 10.4 Similarly, for $\left(k_{i j}, v_{i j}\right)=(2, \infty)$ we use $\hat{\hat{\rho}}$ instead of $\rho_{3}$, Proposition 15.10 instead of Lemma 10.4 and Proposition 15.15] instead of Proposition 8.4. For $k_{i j}=1$ there is nothing to show.

The case $\left(k_{i j}, v_{i j}\right)=(1.5,0)$ is very similar to $\left(k_{i j}, v_{i j}\right)=(2,0)$ (Case II of the proof of Proposition 10.6) and uses straightforward adaptions of Lemma 10.4 and Corollary 8.3

Finally, the cases $\left(k_{i j}, v_{i j}\right)=(1.5,2)$ and $\left(k_{i j}, v_{i j}\right)=(1.5, \infty)$ are again similar to the case $\left(k_{i j}, v_{i j}\right)=$ $(2,1)$ (Case I of the proof of Proposition 10.6): replace $\rho_{3}$ by $\widehat{\rho}$, resp. $\hat{\hat{\rho}}$, Proposition 8.4 by Proposition 14.12, resp. Proposition 15.15 and Lemma 10.4 by Proposition 14.13, resp. Proposition 15.10. 
Proposition 16.12. Let $\sigma_{1}, \sigma_{2}$ be two labellings of $\Pi$. Then the following hold:

(a) $\mathcal{A}\left(\Pi, \sigma_{1}, \mathrm{SO}(2)\right) \cong \mathcal{A}\left(\Pi, \sigma_{2}, \mathrm{SO}(2)\right)$.

(b) $\mathcal{A}\left(\Pi, \sigma_{1}, \kappa, \operatorname{Spin}(2)\right) \cong \mathcal{A}\left(\Pi, \sigma_{2}, \kappa, \operatorname{Spin}(2)\right)$.

Proof. Assertion (a) follows by generalizing the proof of Consequence 9.5, and setting $\alpha_{i j}:=\operatorname{id}_{G_{i j}}$ whenever $a(i, j)$ and $a(j, i)$ have different parities or $\kappa\left(i^{\sigma}\right) \neq \kappa\left(j^{\sigma}\right)$; otherwise, set $\alpha_{i j}:=\mathrm{id}_{G_{i j}}$, if $\pi$ preserves the order relation between $i$ and $j$, and define $\alpha_{i j}$ via $K_{1} \rightarrow K_{2}: D(\phi) \mapsto D(\phi)$ and $K_{2} \rightarrow$ $K_{1}: D(\phi) \mapsto D(\phi)$ in analogy to Lemma 9.4, if $\pi$ switches the order relation between $i$ and $j$. Observe that the permutation $\pi \in \operatorname{Sym}(I)$ with $\pi \sigma_{1}=\sigma_{2}$ preserves $\kappa$, as for $i_{1}, i_{2} \in I$ with $i_{1}^{\sigma_{1}}=i_{2}^{\sigma_{2}}$ certainly $\kappa\left(i_{1}^{\sigma_{1}}\right)=\kappa\left(i_{2}^{\sigma_{2}}\right)$. Therefore, since $\mathcal{A}\left(\Pi, \sigma_{i}, \operatorname{SO}(2)\right)$ induces $\mathcal{A}\left(\Pi, \sigma_{i}, \kappa, \operatorname{Spin}(2)\right)$ for $i=1,2$, assertion (b) is an immediate consequence of (a) and Proposition 16.11 .

We now generalize Definitions 9.6 and 10.8 ,

Definition 16.13. We write $\mathcal{A}(\Pi, \mathrm{SO}(2))$ to denote the isomorphism type of $\mathcal{A}(\Pi, \sigma, \operatorname{SO}(2))$ and $\mathcal{A}(\Pi, \kappa, \operatorname{Spin}(2))$ to denote the isomorphism type of $\mathcal{A}(\Pi, \sigma, \kappa, \operatorname{Spin}(2))$. By slight abuse of notation, we also denote any representative of the respective isomorphism types by these symbols. They are called the standard SO(2)-amalgam with respect to $\Pi$, resp. the standard Spin(2)-amalgam with respect to $\Pi$ and $\kappa$. Accordingly, we denote the epimorphism of amalgams from Remark 16.10 by

$$
\pi_{\Pi, \kappa}: \mathcal{A}(\Pi, \kappa, \operatorname{Spin}(2)) \rightarrow \mathcal{A}(\Pi, \mathrm{SO}(2)) .
$$

Theorems 9.8 and 10.9 generalize from simply laced diagram to arbitrary admissible diagrams.

Theorem 16.14. The following hold:

(a) Any continuous $\mathrm{SO}(2)$-amalgam $\mathcal{A}$ with respect to $\Pi$ and $\sigma$ is isomorphic to the standard amalgam $\mathcal{A}(\Pi, \mathrm{SO}(2))$.

(b) Any continuous $\operatorname{Spin}(2)$-amalgam $\widetilde{\mathcal{A}}$ with respect to $\Pi, \sigma$ and $\kappa$ is isomorphic to the standard amalgam $\mathcal{A}(\Pi, \kappa, \operatorname{Spin}(2))$.

Proof. (a) The following is an adaption of the proof of Consequence 9.8, which will imply the claim via Proposition 16.12. The only continuous automorphisms of the circle group $\mathrm{SO}(2)$ are id and the inversion inv. Since $\mathcal{A}$ continuous by hypothesis, for all $i \neq j \in I$, if $i^{\sigma} \rightarrow j^{\sigma}$, one has

$$
\phi_{i j}^{i} \in\left\{\varepsilon_{1}^{a(i, j), a(j, i)}, \varepsilon_{1}^{a(i, j), a(j, i)} \circ \operatorname{inv}\right\}, \quad \phi_{i j}^{j} \in\left\{\varepsilon_{2}^{a(i, j), a(j, i)}, \varepsilon_{2}^{a(i, j), a(j, i)} \circ \operatorname{inv}\right\} .
$$

For $m=1,2,3$ respectively, set $\gamma_{B}, \gamma_{C}$ as in Lemma 9.7, 14.5 or 13.8 respectively. If $m=0$, then set $\gamma_{B}=\mathrm{inv} \times$ id and $\gamma_{C}=\mathrm{id} \times$ inv. If $m=\infty$, then set $\gamma_{B}$ to be the automorphism induced by the inversion on $K_{1}$ and the identity on $K_{2}$ and $\gamma_{C}$ to be the automorphism induced by the identity on $K_{1}$ and the inversion on $K_{2}$. Using this, for $i^{\sigma} \rightarrow j^{\sigma}$ let

$$
\alpha_{i j}:=\left\{\begin{array}{lll}
\operatorname{id}_{G_{i j}}, & \text { if } \phi_{i j}^{i}=\varepsilon_{1}^{a(i, j), a(j, i)}, & \phi_{i j}^{j}=\varepsilon_{2}^{a(i, j), a(j, i)}, \\
\gamma_{B}, & \text { if } \phi_{i j}^{i}=\varepsilon_{1}^{a(i, j), a(j, i)} \circ \text { inv }, \phi_{i j}^{j}=\varepsilon_{2}^{a(i, j), a(j, i)}, \\
\gamma_{C}, & \text { if } \phi_{i j}^{i}=\varepsilon_{1}^{a(i, j), a(j, i)}, \quad \phi_{i j}^{j}=\varepsilon_{2}^{a(i, j), a(j, i)} \circ \text { inv }, \\
\gamma_{B} \circ \gamma_{C}, & \text { if } \phi_{i j}^{i}=\varepsilon_{1}^{a(i, j), a(j, i)} \circ \text { inv }, \phi_{i j}^{j}=\varepsilon_{2}^{a(i, j), a(j, i)} \circ \text { inv. }
\end{array}\right.
$$

Then the system $\alpha:=\left\{\pi, \alpha_{i j} \mid i \neq j \in I\right\}: \mathcal{A} \rightarrow \mathcal{A}(\Pi, \sigma, \mathrm{SO}(2))$ is an isomorphism of amalgams.

(b) Let $\mathcal{A}$ be the continuous $\mathrm{SO}(2)$-amalgam that induces $\widetilde{\mathcal{A}}$ (cf. Remark16.10). Assertion (a) implies $\mathcal{A} \cong \mathcal{A}(\Pi, \mathrm{SO}(2))$. Proposition 16.11 yields the claim, since $\mathcal{A}(\Pi, \mathrm{SO}(2))$ induces $\mathcal{A}(\Pi, \kappa, \operatorname{Spin}(2))$.

We now generalize Theorem 11.2

Theorem 16.15. Let $G(\Pi)$ be the simply connected split real Kac-Moody group associated to $\Pi$, and let $K(\Pi)$ be its maximal compact subgroup, i.e., the subgroup fixed by the Cartan-Chevalley involution. Then there exists a faithful universal enveloping morphism

$$
\tau_{K(\Pi)}: \mathcal{A}(\Pi, \mathrm{SO}(2)) \rightarrow K(\Pi) .
$$

Proof. The same proof as for Theorem 11.2 applies; the uniqueness of amalgams then follows from Theorem 16.14 (instead of Theorem 9.8).

In view of Theorem 16.15, we generalize Definition 11.5 as follows: 
Definition 16.16. The spin group $\operatorname{Spin}(\Pi, \kappa)$ with respect to $\Pi$ and $\kappa$ is the canonical universal enveloping group of the (continuous) amalgam $\mathcal{A}(\Pi, \kappa, \operatorname{Spin}(2))=\left\{\widetilde{K}_{i j}, \tilde{\phi}_{i j}^{i} \mid i \neq j \in I\right\}$ with the canonical universal enveloping morphism

$$
\tau_{\operatorname{Spin}(\Pi), \kappa}: \mathcal{A}(\Pi, \kappa, \operatorname{Spin}(2)) \rightarrow \operatorname{Spin}(\Pi, \kappa) .
$$

The maximal spin group $\operatorname{Spin}(\Pi)$ with respect to $\Pi$ is $\operatorname{Spin}\left(\Pi, \kappa_{\max }\right)$ (cf. Lemma 16.4).

Observation 16.17. Whenever $\kappa \preceq \kappa^{\prime}$ are admissible colourings of $\Pi$, by construction there exists a canonical central extension

$$
\operatorname{Spin}\left(\Pi, \kappa^{\prime}\right) \rightarrow \operatorname{Spin}(\Pi, \kappa) .
$$

Lemma 16.18. $K(\Pi)$ is an enveloping group of the amalgam $\mathcal{A}(\Pi, \kappa, \operatorname{Spin}(2))$. There exists a canonical central extension $\rho_{\Pi, \kappa}: \operatorname{Spin}(\Pi, \kappa) \rightarrow K(\Pi)$ that makes the following diagram commute:

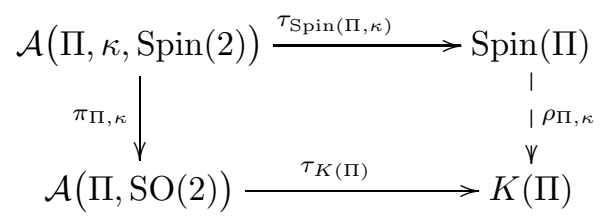

Proof. Essentially the same proof as for Lemma [1.6] works, after appropriately substituting definitions and results from the present Section 16 .

\section{SPIN COVERS OF ARBITRARY TYPE}

In this section we prove the following theorem concerning the central extension $\rho_{\Pi, \kappa}: \operatorname{Spin}(\Pi, \kappa) \rightarrow$ $K(\Pi)$ from Lemma 16.18 .

Theorem 17.1. Given a diagram $\Pi$ and an admissible colouring $\kappa$, the universal enveloping group $\operatorname{Spin}(\Pi, \kappa)$ of $\mathcal{A}(\Pi, \kappa, \operatorname{Spin}(2))$ is a $2^{c(\Pi, \kappa)}$-fold central extension of the universal enveloping group $K(\Pi)$ of $\mathcal{A}(\Pi, \mathrm{SO}(2))$.

Recall from Definition 16.2 that the number $c(\Pi, \kappa)$ counts the connected components of $\Pi^{\text {adm }}$ on which $\kappa$ takes the value 2 .

Remark 17.2. Let $\kappa$ be an admissible colouring of a simply laced diagram $\Pi$. One has $\Pi=\Pi^{\text {adm }}$ and $\kappa$ is constant on each connected component of $\Pi$. The number $c(\Pi, \kappa)$ counts the components on which it has value 2. A reduction to the irreducible case as in the proof of Theorem 11.17 therefore immediately implies Theorem 17.1 for simply laced diagrams.

Our strategy of proof in the general case is based on a reduction to the simply-laced case. We start our investigation with carrying out Strategy 12.2 for doubly laced diagrams.

Definition 17.3. Let $n \in \mathbb{N}$, let $I=\{1, \ldots, n\}$ and let $\Pi$ be an irreducible doubly laced Dynkin diagram that is not simply laced with generalized Cartan matrix $A=(a(i, j))_{i, j}$, labelling $\sigma: I \rightarrow V(\Pi)$ and admissible colouring $\kappa: V \rightarrow\{1,2\}$ such that for each pair of vertices $i^{\sigma}, j^{\sigma}$ with $i^{\sigma} \rightarrow j^{\sigma}$ that form a diagram of type $\mathrm{C}_{2}$ one has $\kappa\left(i^{\sigma}\right)=2$.

Then the unfolded Dynkin diagram is the Dynkin diagram $\Pi^{\text {un }}$ with labelling

$$
\sigma^{\mathrm{un}}: I^{\mathrm{un}}:=\left\{ \pm i \mid i \in I, \kappa\left(i^{\sigma}\right)=1\right\} \cup\left\{i \mid i \in I, \kappa\left(i^{\sigma}\right)=2\right\} \rightarrow V\left(\Pi^{\mathrm{un}}\right)
$$

and edges defined via the generalized Cartan matrix $A^{\text {un }}=\left(a^{\text {un }}(i, j)\right)_{i, j}$ given by

$$
a^{\text {un }}(i, j)= \begin{cases}0, & \text { if } \kappa\left(|i|^{\sigma}\right) \neq \kappa\left(|j|^{\sigma}\right) \text { and } a(|i|,|j|)=0, \\ -1, & \text { if } \kappa\left(|i|^{\sigma}\right) \neq \kappa\left(|j|^{\sigma}\right) \text { and } a(|i|,|j|) \neq 0, \\ a(|i|,|j|), & \text { if } \kappa\left(|i|^{\sigma}\right)=\kappa\left(|j|^{\sigma}\right) \text { and } i j>0, \\ 0, & \text { if } \kappa\left(|i|^{\sigma}\right)=\kappa\left(|j|^{\sigma}\right) \text { and } i j<0 .\end{cases}
$$

Note that the unfolded Dynkin diagram $\Pi^{\text {un }}$ is simply laced. Define the admissible colouring $\kappa^{\text {un }}: \equiv 2$.

For a reducible Dynkin diagram $\Pi$ with admissible colouring $\kappa: V \rightarrow\{1,2\}$ such that for each pair of vertices $i^{\sigma}, j^{\sigma}$ with $i^{\sigma} \rightarrow j^{\sigma}$ that form a diagram of type $\mathrm{C}_{2}$ one has $\kappa\left(i^{\sigma}\right)=2$, define the unfolded Dynkin diagram $\Pi^{\mathrm{un}}$ and its admissible colouring $\kappa^{\mathrm{un}}$ componentwise: replace each connected component that is not simply laced by its unfolded Dynkin diagram and colouring with constant value 2 as defined above and retain each simply laced component and its colouring. 
Remark 17.4. Note that for an irreducible doubly laced Dynkin diagram $\Pi$ that is not simply laced with labelling $\sigma: I \rightarrow V(\Pi)$ there is at most one admissible colouring $\kappa: V \rightarrow\{1,2\}$ such that for each pair of vertices $i^{\sigma}, j^{\sigma}$ with $i^{\sigma} \rightarrow j^{\sigma}$ that form a diagram of type $\mathrm{C}_{2}$ one has $\kappa\left(i^{\sigma}\right)=2$ and, if it exists, actually is equal to the maximal admissible colouring $\kappa_{\text {max }}$ from Lemma 16.4.

This is not necessarily true for reducible such Dynkin diagrams as any simply laced connected components allows the two colourings constant one and constant two.

Proposition 17.5. Let $\Pi$ be a doubly laced Dynkin diagram with labelling $\sigma: I \rightarrow V$ and admissible colouring $\kappa: V \rightarrow\{1,2\}$ such that for each pair of vertices $i^{\sigma}, j^{\sigma}$ with $i^{\sigma} \rightarrow j^{\sigma}$ that form a diagram of type $\mathrm{C}_{2}$ one has $\kappa\left(i^{\sigma}\right)=2$, let $\Pi^{\mathrm{un}}$ be the unfolded Dynkin diagram, let $G(\Pi)$ and $G\left(\Pi^{\mathrm{un}}\right)$ be the corresponding simply connected split real Kac-Moody groups, let $K(\Pi)$ and $K\left(\Pi^{\mathrm{un}}\right)$ be their maximal compact subgroups, let

$$
\tau_{K(\Pi)}: \mathcal{A}(\Pi, \mathrm{SO}(2)) \rightarrow K(\Pi)
$$

and

$$
\tau_{K\left(\Pi^{\mathrm{un}}\right)}: \mathcal{A}\left(\Pi^{\mathrm{un}}, \mathrm{SO}(2)\right) \rightarrow K\left(\Pi^{\mathrm{un}}\right)
$$

be the respective (faithful) universal enveloping morphisms (cf. Theorem 16.15), for each $i \in I$ let

$$
K_{i}:=\left(\tau_{K(\Pi)} \circ \phi_{i j}^{i}\right)(\mathrm{SO}(2)) \leq K(\Pi),
$$

and for each $i \in I^{\text {un }}$ let

$$
K_{i}^{\mathrm{un}}:=\left(\tau_{K\left(\Pi^{\mathrm{un}}\right)} \circ\left(\phi_{i j}^{i}\right)^{\mathrm{un}}\right)(\mathrm{SO}(2)) \leq K\left(\Pi^{\mathrm{un}}\right),
$$

where $\left(\phi_{i j}^{i}\right)^{\mathrm{un}}$ denote the connecting homomorphisms of the amalgam $\mathcal{A}\left(\Pi^{\mathrm{un}}, \mathrm{SO}(2)\right)$.

Then the assignment

$$
\begin{aligned}
& \forall i \in I \text { with } \kappa\left(i^{\sigma}\right)=2: \quad K_{i} \rightarrow K_{i}^{\text {un }} \\
& g \mapsto\left(\tau_{K\left(\Pi^{\text {un }}\right)} \circ\left(\phi_{i j}^{i}\right)^{\text {un }}\right) \circ\left(\tau_{K(\Pi)} \circ \phi_{i j}^{i}\right)^{-1}(g) \\
& \forall i \in I \text { with } \kappa\left(i^{\sigma}\right)=1: \quad K_{i} \rightarrow K_{i}^{\mathrm{un}} \times K_{-i}^{\mathrm{un}} \\
& g \mapsto\left(\left(\tau_{K\left(\Pi^{\mathrm{un}}\right)} \circ\left(\phi_{i j}^{i}\right)^{\mathrm{un}}\right) \times\left(\tau_{K\left(\Pi^{\mathrm{un}}\right)} \circ\left(\phi_{-i j}^{-i}\right)^{\mathrm{un}}\right)\right) \circ\left(\tau_{K(\Pi)} \circ \phi_{i j}^{i}\right)^{-1}(g)
\end{aligned}
$$

induces a monomorphism

$$
\Omega_{K(\Pi)}: K(\Pi) \rightarrow K\left(\Pi^{\mathrm{un}}\right)
$$

Proof. The existence of the homomorphism $\Omega_{K(\Pi)}: K(\Pi) \rightarrow K\left(\Pi^{\mathrm{un}}\right)$ is straightforward using Consequence 14.11 and the universal property of $\tau_{K(\Pi)}: \mathcal{A}(\Pi, \mathrm{SO}(2)) \rightarrow K(\Pi)$. Injectivity follows from the faithfulness of the universal enveloping morphism $\tau_{K\left(\Pi^{\mathrm{un}}\right)}: \mathcal{A}\left(\Pi^{\mathrm{un}}, \mathrm{SO}(2)\right) \rightarrow K\left(\Pi^{\mathrm{un}}\right)$.

Corollary 17.6. Retain the notation and hypotheses from Proposition 17.5. Moreover, let

$$
\tau_{\operatorname{Spin}(\Pi, \kappa)}: \mathcal{A}(\Pi, \kappa, \operatorname{Spin}(2)) \rightarrow \operatorname{Spin}(\Pi, \kappa)
$$

and

$$
\tau_{\mathrm{Spin}\left(\Pi^{\mathrm{un}}, \kappa^{\mathrm{un}}\right)}: \mathcal{A}\left(\Pi^{\mathrm{un}}, \kappa^{\mathrm{un}}, \operatorname{Spin}(2)\right) \rightarrow \operatorname{Spin}\left(\Pi^{\mathrm{un}}, \kappa^{\mathrm{un}}\right)
$$

be the respective universal enveloping morphisms (cf. Definitions 11.5 and 16.16), for each $i \in I$ let

$$
\widetilde{K}_{i}:=\left(\tau_{\operatorname{Spin}(\Pi, \kappa)} \circ \widetilde{\phi}_{i j}^{i}\right)(\operatorname{Spin}(2)) \leq \operatorname{Spin}(\Pi, \kappa),
$$

and for each $i \in I^{\text {un }}$ let

$$
\widetilde{K}_{i}^{\mathrm{un}}:=\left(\tau_{\operatorname{Spin}\left(\Pi^{\mathrm{un}}, \kappa^{\mathrm{un}}\right)} \circ\left(\widetilde{\phi}_{i j}^{i}\right)^{\mathrm{un}}\right)(\operatorname{Spin}(2)) \leq \operatorname{Spin}\left(\Pi^{\mathrm{un}}, \kappa^{\mathrm{un}}\right),
$$

where $\left(\widetilde{\phi}_{i j}^{i}\right)^{\mathrm{un}}$ denote the connecting homomorphisms of the amalgam $\mathcal{A}\left(\Pi^{\mathrm{un}}, \kappa^{\mathrm{un}}, \operatorname{Spin}(2)\right)$.

Then the assignment

$\forall i \in I$ with $\kappa\left(i^{\sigma}\right)=2: \quad \widetilde{K}_{i} \rightarrow \widetilde{K}_{i}^{\text {un }}$

$$
g \mapsto\left(\tau_{\operatorname{Spin}\left(\Pi^{\mathrm{un}}, \kappa^{\mathrm{un}}\right)} \circ\left(\widetilde{\phi}_{i j}^{i}\right)^{\mathrm{un}}\right) \circ\left(\tau_{\operatorname{Spin}(\Pi, \kappa)} \circ \widetilde{\phi}_{i j}^{i}\right)^{-1}(g)
$$

$\forall i \in I$ with $\kappa\left(i^{\sigma}\right)=1: \quad \widetilde{K}_{i} \rightarrow \widetilde{K}_{i}^{\text {un }} \cdot \widetilde{K}_{-i}^{\text {un }}$

$$
g \mapsto\left(\left(\tau_{\operatorname{Spin}\left(\Pi^{\mathrm{un}}, \kappa^{\mathrm{un}}\right)} \circ\left(\widetilde{\phi}_{i j}^{i}\right)^{\mathrm{un}}\right) \cdot\left(\tau_{\operatorname{Spin}\left(\Pi_{\mathrm{un}}, \kappa^{\mathrm{un}}\right)} \circ\left(\widetilde{\phi}_{-i j}^{-i}\right)^{\mathrm{un}}\right)\right) \circ\left(\tau_{\operatorname{Spin}(\Pi, \kappa)} \circ \widetilde{\phi}_{i j}^{i}\right)^{-1}(g)
$$


induces a homomorphism $\Omega_{\operatorname{Spin}(\Pi, \kappa)}: \operatorname{Spin}(\Pi, \kappa) \rightarrow \operatorname{Spin}\left(\Pi^{\mathrm{un}}, \kappa^{\mathrm{un}}\right)$ that makes the following diagram commute:

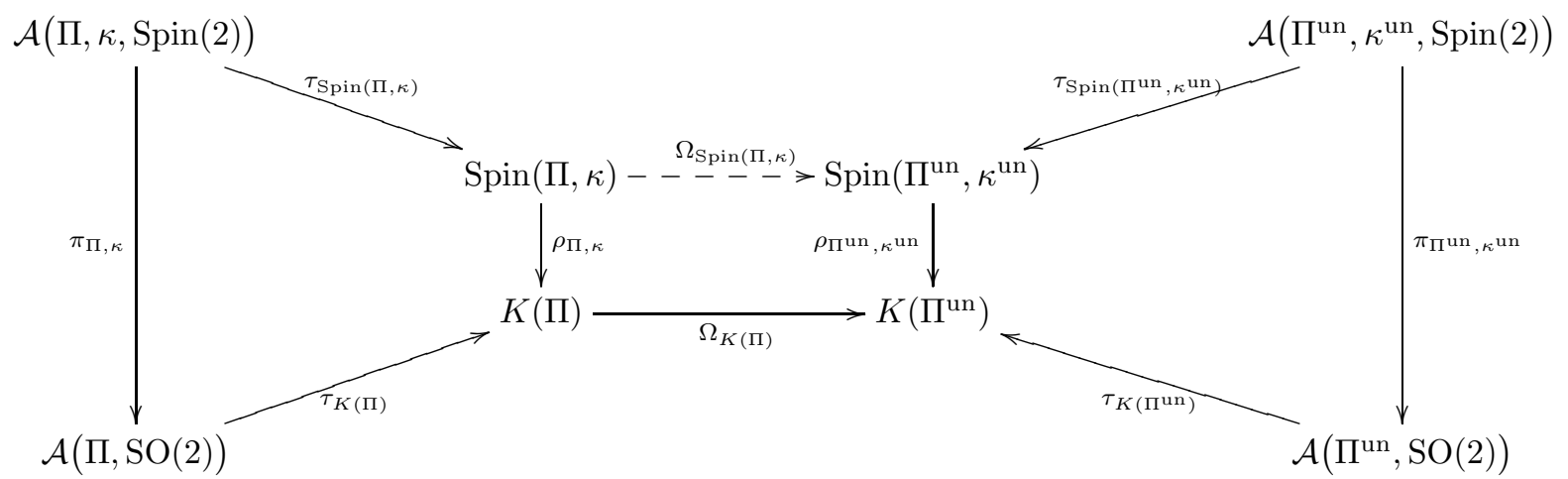

In particular, if the admissible colouring $\kappa$ is non-trivial, then

$$
\rho_{\Pi, \kappa}: \operatorname{Spin}(\Pi, \kappa) \rightarrow K(\Pi)
$$

is a non-trivial central extension.

Proof. The existence of the homomorphism $\Omega_{\operatorname{Spin}(\Pi, \kappa)}: \operatorname{Spin}(\Pi, \kappa) \rightarrow \operatorname{Spin}\left(\Pi^{\mathrm{un}}, \kappa^{\mathrm{un}}\right)$ is straightforward using Lemma 14.9 and the universal property of $\tau_{\operatorname{Spin}(\Pi), \kappa}: \mathcal{A}(\Pi, \kappa, \operatorname{Spin}(2)) \rightarrow \operatorname{Spin}(\Pi, \kappa)$.

Let $i \in I$ with $\kappa\left(i^{\sigma}\right)=2$ and define

$$
\begin{aligned}
z_{i} & :=\left(\tau_{\operatorname{Spin}(\Pi, \kappa)} \circ \widetilde{\phi}_{i j}^{i}\right)(S(\pi)) \in \widetilde{K}_{i}, \\
z_{i}^{\mathrm{un}} & :=\left(\tau_{\operatorname{Spin}\left(\Pi^{\mathrm{un}}, \kappa^{\mathrm{un}}\right)} \circ\left(\widetilde{\phi}_{i j}^{i}\right)^{\mathrm{un}}\right)(S(\pi)) \in \widetilde{K}_{i}^{\mathrm{un}} .
\end{aligned}
$$

Then $\Omega_{\operatorname{Spin}(\Pi, \kappa)}\left(z_{i}\right)=z_{i}^{\text {un }}=-1_{\operatorname{Spin}\left(\Pi^{\text {un }}, \kappa^{\text {un }}\right), \mathcal{K}(i)}$ (cf. Definition 11.8). Since the central extension $\rho_{\Pi^{\text {un }}}$ : $\operatorname{Spin}\left(\Pi^{\mathrm{un}}, \kappa^{\mathrm{un}}\right) \rightarrow K\left(\Pi^{\mathrm{un}}\right)$ is non-trivial by the simply-laced version of Theorem 17.1 (see also Corollary 11.16), so is the central extension $\rho_{\Pi, \kappa}: \operatorname{Spin}(\Pi, \kappa) \rightarrow K(\Pi)$.

Next we carry out Strategy 12.1 in order to reduce arbitrary diagrams to doubly laced ones.

Definition 17.7. For an augmented Dynkin diagram $\Pi$ with labelling $\sigma: I \rightarrow V$ and an admissible colouring $\kappa$, let $\Pi^{\mathrm{dl} \kappa}$ be the doubly laced diagram with identical orientation (cf. Remark 16.7) obtained by

- removing all edges $\{i, j\}^{\sigma} \in E_{\infty}(\Pi)$ with $a(i, j), a(j, i)$ even,

- replacing all edges $\{i, j\}^{\sigma} \in E_{\infty}(\Pi)$ with $a(i, j)$ odd, $a(j, i)$ even and $\kappa\left(i^{\sigma}\right)=2$ by double edges,

- retaining all edges $\{i, j\}^{\sigma} \in E_{2}(\Pi)$ with $a(i, j)$ odd, $a(j, i)$ even and $\kappa\left(i^{\sigma}\right)=2$,

- replacing all other edges in $\Pi$ by simple edges.

Remark 17.8. The diagram $\Pi^{\mathrm{d} 1} \kappa$ is a doubly laced with admissible colouring $\kappa$ such that for each pair of vertices $i^{\sigma}, j^{\sigma}$ with $i^{\sigma} \rightarrow j^{\sigma}$ that form a diagram of type $\mathrm{C}_{2}$ one has $\kappa\left(i^{\sigma}\right)=2$.

Note that $c(\Pi, \kappa)=c\left(\Pi^{\mathrm{dl} \kappa}, \kappa\right)$.

The orientation of $\Pi^{\mathrm{dl} \kappa}$ induced by its labelling and colouring in general differs from the one that $\Pi^{\mathrm{dl} \kappa}$ inherits from the orientation of $\Pi$ induced by its labelling and colouring. Note that, of course, it is possible to change the labellings of $\Pi$ and $\Pi^{\mathrm{d} l \kappa}$ so that the two orientations coincide.

The correspondence of the indices $i, j$ of the epimorphisms $\alpha_{i j}$ and $\tilde{\alpha}_{i j}$ in the following proposition to the indices $p$ and $l$ in Definition 16.9 depends on the latter orientation of $\Pi^{\mathrm{d} l \kappa}$ not the former.

Proposition 17.9. Given a diagram $\Pi$ and an admissible colouring $\kappa$, there exist epimorphisms of amalgams $\tilde{\alpha}=\left(\mathrm{id}, \tilde{\alpha}_{i j}\right): \mathcal{A}(\Pi, \kappa, \operatorname{Spin}(2)) \rightarrow \mathcal{A}\left(\Pi^{\mathrm{dl} \kappa}, \kappa, \operatorname{Spin}(2)\right)$ and $\alpha=\left(\mathrm{id}, \alpha_{i j}\right): \mathcal{A}(\Pi, \mathrm{SO}(2)) \rightarrow$ $\mathcal{A}\left(\Pi^{\mathrm{dl} \kappa}, \mathrm{SO}(2)\right)$ making the following diagram commute:

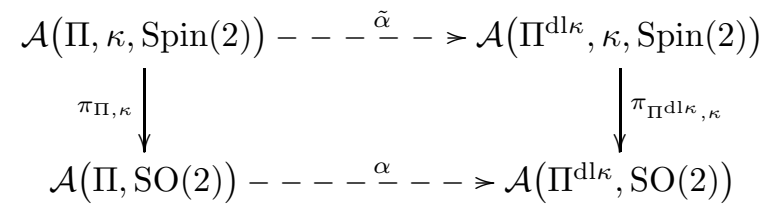


Proof. There exist the following group epimorphisms that provide the epimorphism $\alpha$ :

$$
\begin{aligned}
\mathrm{U}_{2}(\mathbb{C}) & \rightarrow \mathrm{SO}(3) \\
\mathrm{SO}(4) & \rightarrow \mathrm{SO}(3) \\
K\left(A_{1}\right) & \rightarrow \mathrm{SO}(2) \times \mathrm{SO}(2) \\
K\left(A_{2}\right) & \rightarrow \mathrm{SO}(3) \\
K\left(A_{3}\right) & \rightarrow \mathrm{U}_{2}(\mathbb{C})
\end{aligned}
$$

$$
\begin{array}{r}
\text { (see Corollary 14.14) } \\
\text { (see Lemma 13.14) } \\
\text { (see Proposition 15.7) } \\
\text { (see Proposition 15.7) } \\
\text { (see Proposition 15.7) }
\end{array}
$$

where $A_{1}=\left(\begin{array}{cc}2 & -r_{1} \\ -s_{1} & 2\end{array}\right)$ with $r_{1}, s_{1}$ even, $A_{2}=\left(\begin{array}{cc}2 & -r_{2} \\ -s_{2} & 2\end{array}\right)$ with $r_{2}, s_{2}$ odd, $A_{3}=\left(\begin{array}{cc}2 & -r_{3} \\ -s_{3} & 2\end{array}\right)$ with one of $r_{3}, s_{3}$ even, the other odd. The respective lifts to the spin covers exist by Corollary 14.14, Proposition 13.15, Corollary 15.10, yielding the epimorphism $\tilde{\alpha}$. By 13.14, 13.15] and 14.14 and 15.7, 15.10 the above diagram commutes.

Proposition 17.10. Given a diagram $\Pi$ and an admissible colouring $\kappa$, there exist (uniquely determined) epimorphisms

$$
\Xi_{\mathrm{Spin}(\Pi, \kappa)}: \operatorname{Spin}(\Pi, \kappa) \rightarrow \operatorname{Spin}\left(\Pi^{\mathrm{dl} \kappa}, \kappa\right) \quad \text { and } \quad \Xi_{K(\Pi)}: K(\Pi) \rightarrow K\left(\Pi^{\mathrm{dl} \kappa}\right)
$$

resulting in the following commutative diagram:

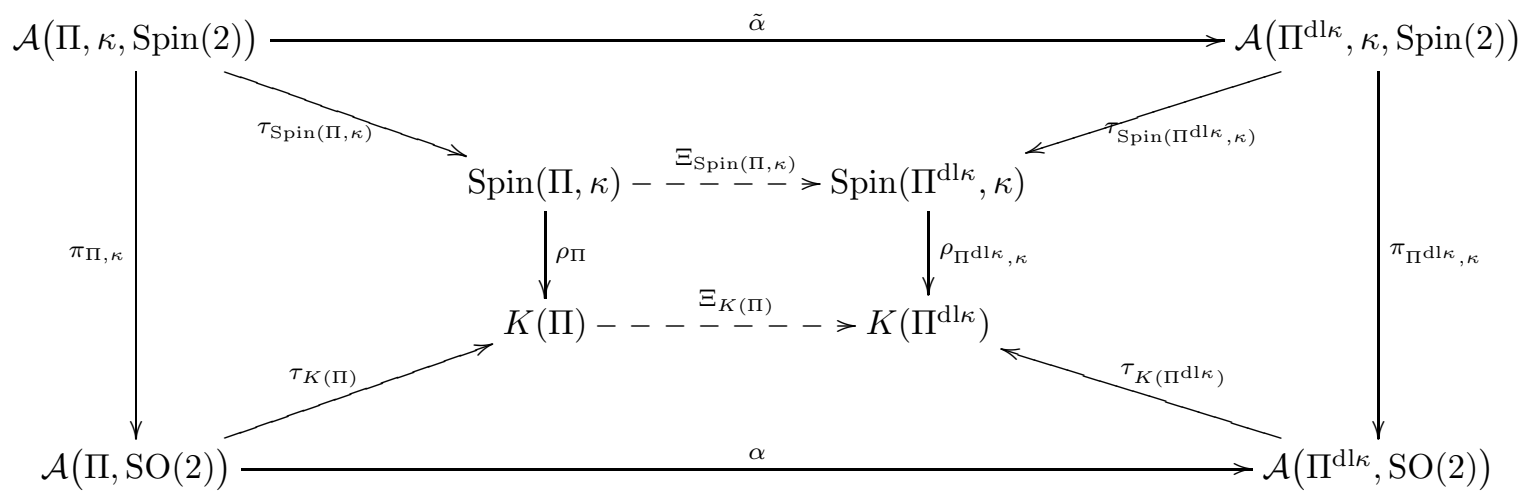

Proof. The left-hand and the right-hand commutative squares exist by Lemma 16.18, The outer commutative square exists by Proposition 17.9. The composition

$$
\tau_{\operatorname{Spin}\left(\Pi^{\mathrm{d} l \kappa}, \kappa\right)} \circ \tilde{\alpha}: \mathcal{A}(\Pi, \kappa, \operatorname{Spin}(2)) \rightarrow \operatorname{Spin}\left(\Pi^{\mathrm{dl} \kappa}, \kappa\right)
$$

is an enveloping morphism, whence the universal property of $\operatorname{Spin}(\Pi, \kappa)$ yields the uniquely determined epimorphism $\Xi_{\operatorname{Spin}(\Pi), \kappa}: \operatorname{Spin}(\Pi, \kappa) \rightarrow \operatorname{Spin}\left(\Pi^{\mathrm{dl} \kappa}, \kappa\right)$, which makes the upper square commute. Similarly, one obtains the uniquely determined epimorphism $\Xi_{K(\Pi)}: K(\Pi) \rightarrow K\left(\Pi^{\mathrm{dl} \kappa}\right)$, that makes the lower square commute. Also

$$
\tau_{K\left(\Pi^{\mathrm{dl} \kappa}\right)} \circ \alpha \circ \pi_{\Pi, \kappa}=\tau_{K\left(\Pi^{\mathrm{dl} \kappa}\right), \kappa} \circ \pi_{\Pi^{\mathrm{dl} \kappa}, \kappa} \circ \tilde{\alpha}: \mathcal{A}(\Pi, \kappa, \operatorname{Spin}(2)) \rightarrow K\left(\Pi^{\mathrm{dl} \kappa}\right)
$$

is an enveloping morphism. By the universal property of $\tau_{\operatorname{Spin}(\Pi, \kappa)}: \mathcal{A}(\Pi, \kappa, \operatorname{Spin}(2)) \rightarrow \operatorname{Spin}(\Pi, \kappa)$ in connection with the outer commuting squares one has $\rho_{\Pi^{\mathrm{dl} \kappa}, \kappa} \circ \Xi_{\operatorname{Spin}(\Pi), \kappa}=\Xi_{K(\Pi)} \circ \rho_{\Pi, \kappa}$. That is, also the inner square commutes.

Proof of Theorem 17.1. Lemma 16.18 provides a canonical central extension $\rho_{\Pi, \kappa}: \operatorname{Spin}(\Pi, \kappa) \rightarrow K(\Pi)$. By Proposition 17.10, there exist an epimorphism $\Xi_{\operatorname{Spin}(\Pi, \kappa)}: \operatorname{Spin}(\Pi, \kappa) \rightarrow \operatorname{Spin}\left(\Pi^{\mathrm{d} l \kappa}, \kappa\right)$ resulting in the commutative diagram:

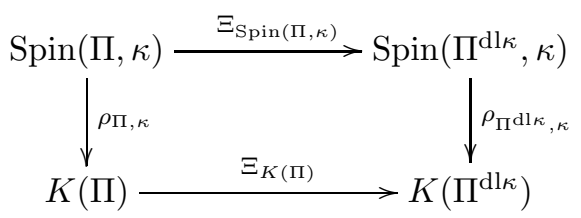

Observe first that the claim is obvious if $\kappa$ is trivial (i.e., $c(\Pi, \kappa)=0$ ). Moreover, it is also true if $\kappa$ is elementary (i.e., $c(\Pi, \kappa)=1$ ). Indeed, since $\operatorname{ker}\left(\rho_{\Pi^{\mathrm{dl} \kappa}, \kappa}\right) \neq\{1\}$ by Corollary 17.6, necessarily also $\operatorname{ker}\left(\rho_{\Pi, \kappa}\right) \neq\{1\}$ by the homomorphism theorem of groups. By Remark 15.12 each connected component of $\Pi^{\text {adm }}$ contributes at most a factor 2 to the order of $\operatorname{ker}\left(\rho_{\Pi, \kappa}\right)$. Since only those connected components that admit a vertex $i^{\sigma}$ with $\kappa\left(i^{\sigma}\right)=2$ actually can contribute, one has $\left|\operatorname{ker}\left(\rho_{\Pi, \kappa}\right)\right| \leq 2^{c(\Pi, \kappa)}$. The claim follows for elementary colourings. 
Since $c(\Pi, \kappa)=c\left(\Pi^{\mathrm{dl} \kappa}, \kappa\right)$, once more the homomorphism theorem of groups implies that is suffices to show the claim for the central extension

$$
\rho_{\Pi^{\mathrm{d} l \kappa}, \kappa}: \operatorname{Spin}\left(\Pi^{\mathrm{dl} \kappa}, \kappa\right) \rightarrow K\left(\Pi^{\mathrm{dl} \kappa}\right)
$$

Since the upper bound that has just been established also holds for $\left|\operatorname{ker}\left(\rho_{\Pi^{\mathrm{dl} \kappa}, \kappa}\right)\right|$, it suffices to prove $2^{c\left(\Pi^{\mathrm{dl} \kappa}, \kappa\right)} \leq\left|\operatorname{ker}\left(\rho_{\Pi^{\mathrm{dl} \kappa}, \kappa}\right)\right|$. To this end decompose $\kappa=\kappa_{1}+\cdots+\kappa_{c\left(\Pi^{\mathrm{d} 1 \kappa}, \kappa\right)}$ into a sum of pairwise distinct elementary admissible colourings of $\Pi^{\mathrm{dl} \kappa}$. Let

$$
\left\{i_{t} \in I \mid 1 \leq t \leq c\left(\Pi^{\mathrm{dl} \kappa}, \kappa\right), \kappa_{t}\left(i_{t}\right)=2\right\}
$$

be a set of representatives of the connected components of $\left(\Pi^{\mathrm{dl} \kappa}\right)^{\text {adm }}$ on which $\kappa$ takes value 2 . By Observation 16.17. for each $1 \leq t \leq c\left(\Pi^{\mathrm{dl} \kappa}, \kappa\right)$ there exists a central extension

$$
\pi_{t}: \operatorname{Spin}\left(\Pi^{\mathrm{dl} \kappa}, \kappa\right) \rightarrow \operatorname{Spin}\left(\Pi^{\mathrm{dl} \kappa}, \kappa_{t}\right) .
$$

Since for each $1 \leq t \leq c\left(\Pi^{\mathrm{dl} \kappa}, \kappa\right)$ the colouring $\kappa_{t}$ is elementary, the central extension

$$
\rho_{\Pi^{\mathrm{d} l \kappa}, \kappa_{t}}: \operatorname{Spin}\left(\Pi^{\mathrm{dl} \kappa}, \kappa_{t}\right) \rightarrow K\left(\Pi^{\mathrm{dl} \kappa}\right)
$$

has kernel of order two. The kernel of the natural homomorphism

$$
\operatorname{Spin}\left(\Pi^{\mathrm{d} l \kappa}, \kappa\right) \longrightarrow \prod_{t=1}^{c\left(\Pi^{\mathrm{d} l \kappa}, \kappa\right)} \operatorname{Spin}\left(\Pi^{\mathrm{d} l \kappa}, \kappa_{t}\right) \longrightarrow \prod_{t=1}^{c\left(\Pi^{\mathrm{d} l \kappa}, \kappa\right)} K\left(\Pi^{\mathrm{d} l \kappa}\right)
$$

equals $\operatorname{ker}\left(\rho_{\Pi^{\mathrm{dl} \kappa}, \kappa}\right)$. By the proof of Corollary 17.6 one has $\pi_{t}\left(z_{i_{s}}\right) \neq 1$ in $\operatorname{Spin}\left(\Pi^{\mathrm{dl} \kappa}, \kappa_{t}\right)$ if and only if $s=t$. We conclude that

$$
2^{c\left(\Pi^{\mathrm{dl} \kappa}, \kappa\right)} \leq\left|\left\langle z_{i_{t}} \mid 1 \leq t \leq c\left(\Pi^{\mathrm{dl} \kappa}, \kappa\right)\right\rangle\right| \leq\left|\operatorname{ker}\left(\rho_{\Pi^{\mathrm{dl} \kappa}, \kappa}\right)\right| .
$$

The claim follows.

\section{Part V. Applications}

\section{Spin-extended Weyl groups}

Let $A$ be a generalized Cartan matrix with corresponding augmented Dynkin diagram $\Pi:=\Pi(A)$. In this section we formally construct the spin cover $W^{\operatorname{spin}}(\Pi, \kappa)$ whose existence has been postulated in [DH09, Section 3.5], for arbitrary diagrams $\Pi$ with admissible colouring $\kappa$ (cf. Definition [16.2). In fact, we provide both a concrete embedding of $W^{\operatorname{spin}}(\Pi, \kappa)$ into $\operatorname{Spin}(\Pi, \kappa)$ and a presentation by generators and relations.

Notation 18.1. Throughout this section, let $\Pi$ be an augmented Dynkin diagram with vertex set $V$, labelling $\sigma: I \rightarrow V$ and admissible colouring $\kappa: V \rightarrow\{1,2\}$. Let $A=(a(i, j))_{i, j \in I}$ be the generalized Cartan matrix associated to $\Pi$. Let $n:=|V|$, i.e., $I=\{1, \ldots, n\}$, and let

$$
J:=\left\{i \in I \mid \kappa\left(i^{\sigma}\right)=1\right\} \subset I .
$$

Definition 18.2. For $i \neq j \in I$, recall from 16.2

$$
n(i, j)= \begin{cases}0, & \text { if } a(i, j) \text { is even } \\ 1, & \text { if } a(i, j) \text { is odd }\end{cases}
$$

Define

$$
m_{i j}:= \begin{cases}2, & \text { if } a(i, j) a(j, i)=0, \\ 3, & \text { if } a(i, j) a(j, i)=1, \\ 4, & \text { if } a(i, j) a(j, i)=2, \\ 6, & \text { if } a(i, j) a(j, i)=3, \\ 0, & \text { if } a(i, j) a(j, i) \geq 4 .\end{cases}
$$

Definition 18.3. Consider the standard amalgams with respect to $\Pi$ and $\kappa$

$$
\mathcal{A}(\Pi, \kappa, \operatorname{Spin}(2))=\left\{\widetilde{G}_{i j}, \tilde{\phi}_{i j}^{i} \mid i \neq j \in I\right\} \quad \text { and } \quad \mathcal{A}(\Pi, \operatorname{SO}(2))=\left\{G_{i j}, \phi_{i j}^{i} \mid i \neq j \in I\right\}
$$

with enveloping homomorphisms $\tilde{\psi}_{i j}: \widetilde{G}_{i j} \rightarrow \operatorname{Spin}(\Pi, \kappa)$ and $\psi_{i j}: G_{i j} \rightarrow K(\Pi)$ to the respective universal enveloping groups.

For $i \neq j \in I$ let

$$
\widehat{s}_{i}:= \begin{cases}\tilde{\psi}_{i j}\left(\tilde{\phi}_{i j}^{i}\left(S\left(\frac{\pi}{4}\right)\right)\right), & \text { if } i \notin J, \\ \tilde{\psi}_{i j}\left(\tilde{\phi}_{i j}^{i}\left(S\left(\frac{\pi}{4}\right)\right)\right)^{2}=\tilde{\psi}_{i j}\left(\tilde{\phi}_{i j}^{i}\left(S\left(\frac{\pi}{2}\right)\right)\right), & \text { if } i \in J\end{cases}
$$


and set

Similarly, let

$$
\widehat{W}:=\widehat{W}(\Pi, \kappa):=\left\langle\widehat{s}_{i} \mid i \in I\right\rangle \leq \operatorname{Spin}(\Pi, \kappa) .
$$

$$
\widetilde{s}_{i}:=\psi_{i j}\left(\phi_{i j}^{i}\left(D\left(\frac{\pi}{2}\right)\right)\right) \quad \text { and } \quad \widetilde{W}:=\widetilde{W}(\Pi):=\left\langle\widetilde{s}_{i} \mid i \in I\right\rangle \leq K(\Pi) .
$$

Note that the elements $\widehat{s}_{i}$ and $\widetilde{s}_{i}$ are well-defined — in particular, independent of the choice of $j$ due to the definition of enveloping groups.

Definition 18.4. The Weyl group $\boldsymbol{W}(\Pi)$ associated to $\Pi$ is given by the finite presentation

$$
\begin{aligned}
& W:=W(\Pi):=\left\langle s_{1}, \ldots, s_{n}\right| s_{i}^{2}=1 \text { for } i \\
&\qquad \underbrace{s_{i} s_{j} s_{i} \cdots}_{m_{i j} \text { factors }}=\underbrace{s_{j} s_{i} s_{j} \cdots}_{m_{i j} \text { factors }} \text { for } i \neq j \in I\rangle .
\end{aligned}
$$

The extended Weyl group $\boldsymbol{W}^{\mathbf{e x t}}(\Pi)$ associated to $\Pi$ is given by the finite presentation

$$
\begin{aligned}
& W^{\mathrm{ext}}:=W^{\operatorname{ext}}(\Pi):=\left\langle t_{1}, \ldots, t_{n}\right| t_{i}^{4}=1 \text { for } i \in I, \\
& \qquad t_{j}^{-1} t_{i}^{2} t_{j}=t_{i}^{2} t_{j}^{2 n(i, j)} \text { for } i \neq j \in I, \\
& \underbrace{t_{i} t_{j} t_{i} \cdots}_{m_{i j} \text { factors }}=\underbrace{t_{j} t_{i} t_{j} \cdots}_{m_{i j} \text { factors }} \text { for } i \neq j \in I\rangle .
\end{aligned}
$$

The spin-extended Weyl group $\boldsymbol{W}^{\text {spin }}(\Pi)$ associated to $\Pi$ is given by the finite presentation

$$
\begin{aligned}
W^{\text {spin }}:=W^{\operatorname{spin}}(\Pi):=\left\langle r_{1}, \ldots, r_{n}\right| & r_{i}^{8}=1, \text { for } i \in I, \\
& r_{j}^{-1} r_{i}^{2} r_{j}=r_{i}^{2} r_{j}^{2 n(i, j)} \quad \text { for } i \neq j \in I, \\
& \underbrace{r_{i} r_{j} r_{i} \cdots}_{m_{i j} \text { factors }}=\underbrace{r_{j} r_{i} r_{j} \cdots}_{m_{i j} \text { factors }} \text { for } i \neq j \in I\rangle .
\end{aligned}
$$

Remark 18.5. The group $W^{\operatorname{ext}}(\Pi)$ is studied in [Tit66] and KP85. By [KP85, Corollary 2.4] and its proof there exists an isomorphism

$$
W^{\mathrm{ext}}(\Pi) \cong \widetilde{W}(\Pi)
$$

induced by $t_{i} \mapsto \widetilde{s}_{i}$. The elements $\left(\widetilde{s}_{i}\right)^{2}$ are torus elements of $K(\Pi)$ of order two, so that the relation (T2)

$$
t_{j}^{-1} t_{i}^{2} t_{j}=t_{i}^{2} t_{j}^{2 n(i, j)} \quad \Longleftrightarrow \quad t_{i}^{-2} t_{j}^{-1} t_{i}^{2}=t_{j}^{2 n(i, j)} t_{j}^{-1}
$$

is equivalent to the centralize-or-invert relation (11) for torus elements of order two discussed in Remark 15.4. If one prefers left-conjugation, one can therefore use one of the relations

$$
\begin{aligned}
t_{j} t_{i}^{2} t_{j}^{-1}=t_{i}^{2} t_{j}^{-2 n(i, j)} & \Longleftrightarrow \quad t_{i}^{-2} t_{j} t_{i}^{2}=t_{j}^{-2 n(i, j)} t_{j} \\
t_{j} t_{i}^{2} t_{j}^{-1}=t_{j}^{2 n(i, j)} t_{i}^{2} & \Longleftrightarrow \quad t_{i}^{2} t_{j}^{-1} t_{i}^{-2}=t_{j}^{-1} t_{j}^{2 n(i, j)}
\end{aligned}
$$

instead of (T2).

This accounts for the missing minus sign in relation (T2) of our presentation of $W^{\text {ext }}(\Pi)$ compared to relation (n1) given in [KP85, Corollary 2.4]. Since, because of relation (T1) $t_{i}^{4}=1$, only the parity of $a(i, j)$ matters, there is no harm in replacing $a(i, j)$ in relation (n1) from [KP85, Corollary 2.4] with $n(i, j)$, as we have done in our relation (T2).

The version of the relations of $W^{\text {ext }}(\Pi)$ we chose can then be directly generalized - simply by weakening relation (T1) to (R1) - in order to obtain suitable relations of $W^{\text {spin }}(\Pi)$ to make it the appropriate spinextended Weyl group for the spin group $\operatorname{Spin}(\Pi)$. Valid alternatives for relation (R2) are

$$
\begin{aligned}
r_{j} r_{i}^{2} r_{j}^{-1}=r_{i}^{2} r_{j}^{-2 n(i, j)} & \Longleftrightarrow r_{i}^{-2} r_{j} r_{i}^{2}=r_{j}^{-2 n(i, j)} r_{j} \\
r_{j} r_{i}^{2} r_{j}^{-1}=r_{j}^{2 n(i, j)} r_{i}^{2} & \Longleftrightarrow \quad r_{i}^{2} r_{j}^{-1} r_{i}^{-2}=r_{j}^{-1} r_{j}^{2 n(i, j)}
\end{aligned}
$$

as all are equivalent to the centralize-or-invert relation (2) from Proposition 15.10,

Lemma 18.6. In $W^{\text {spin }}(\Pi)$ for all $i, j \in I$ the following relations hold:

(a) $\left[r_{i}^{2}, r_{j}^{2}\right]=r_{j}^{4 n(i, j)}$ and $r_{i}^{4 n(j, i)}=r_{j}^{4 n(i, j)}$, 
(b) if $n(i, j)=1=n(i, j)$, then $r_{i}^{4}=r_{j}^{4}$; otherwise $\left[r_{i}^{2}, r_{j}^{2}\right]=1$,

(c) if $n(i, j)=0, n(j, i)=1$, then $r_{i}^{4}=1$,

(d) $r_{j} r_{i}^{4} r_{j}^{-1}= \begin{cases}r_{i}^{4} & \text { if } n(i, j)=0, \\ r_{i}^{2} r_{j}^{2} r_{i}^{2} r_{j}^{2} & \text { if } n(i, j)=1,\end{cases}$

(e) $\left[r_{j}, r_{i}^{4}\right]=1$.

Proof. One computes

$$
\begin{aligned}
& r_{j}^{-1} r_{i}^{2} r_{j}=r_{i}^{2} r_{j}^{2 n(i, j)} \\
\Longleftrightarrow & r_{j}^{-2} r_{i}^{2} r_{j}^{2}=r_{j} r_{i}^{2} r_{j}^{-1+2 n(i, j)}=r_{i}^{2} r_{j}^{2 n(i, j)} r_{j}^{2 n(i, j)} \\
& {\left[r_{i}^{2}, r_{j}^{2}\right]=r_{j}^{4 n(i, j)} }
\end{aligned}
$$

Moreover:

$$
r_{i}^{4 n(j, i)}=r_{i}^{-4 n(j, i)}=\left[r_{j}^{2}, r_{i}^{2}\right]^{-1}=\left[r_{i}^{2}, r_{j}^{2}\right]=r_{j}^{4 n(i, j)} .
$$

If $n(i, j)=1=n(j, i)$ this immediately implies $r_{i}^{4}=r_{j}^{4}$. Furthermore, if one of $n(i, j), n(j, i)$ is 0 , then each term in (3) is equal to 1 , in particular $\left[r_{i}^{2}, r_{j}^{2}\right]=1$. In case $n(i, j)=0$ and $n(j, i)=1$ one obtains $r_{i}^{4}=r_{i}^{4 n(j, i)}=r_{j}^{4 n(i, j)}=1$.

Also:

$$
r_{j}^{-1} r_{i}^{4} r_{j}=\left(r_{j}^{-1} r_{i}^{2} r_{j}\right)^{2}=\left(r_{i}^{2} r_{j}^{2 n(i, j)}\right)^{2}= \begin{cases}r_{i}^{4} & \text { if } n(i, j)=0, \\ r_{i}^{2} r_{j}^{2} r_{i}^{2} r_{j}^{2} & \text { if } n(i, j)=1 .\end{cases}
$$

If $n(i, j)=1$ and $n(j, i)=0$, then $\left[r_{i}^{2}, r_{j}^{2}\right]=1$ and $r_{j}^{4}=1$, whence $r_{j}^{-1} r_{i}^{4} r_{j}=r_{i}^{2} r_{j}^{2} r_{i}^{2} r_{j}^{2}=r_{i}^{4} r_{j}^{4}=r_{i}^{4}$. Finally, if $n(i, j)=1=n(j, i)$, then $r_{i}^{4}=r_{j}^{4}$, so we also have $r_{j}^{-1} r_{i}^{4} r_{j}=r_{j}^{-1} r_{j}^{4} r_{j}=r_{j}^{4}=r_{i}^{4}$. Therefore always $\left[r_{j}, r_{i}^{4}\right]=1$.

Consequence 18.7. The subgroup $Z:=\left\langle r_{i}^{4} \mid i \in I\right\rangle$ of $W^{\operatorname{spin}}(\Pi)$ is central.

Definition 18.8. The coloured spin-extended Weyl group $\boldsymbol{W}^{\operatorname{spin}}(\Pi, \kappa)$ associated to $\Pi$ and $\kappa$ is given by the finite presentation

$$
\begin{aligned}
& W^{\operatorname{spin}}(\Pi, \kappa):=\left\langle r_{1}, \ldots, r_{n}\right| \\
& r_{i}^{8}=1, \text { for } i \in I, \\
& r_{j}^{-1} r_{i}^{2} r_{j}=r_{i}^{2} r_{j}^{2 n(i, j)} \text { for } i \neq j \in I, \\
& \underbrace{r_{i} r_{j} r_{i} \cdots}_{m_{i j} \text { factors }}=\underbrace{r_{j} r_{i} r_{j} \cdots}_{m_{i j} \text { factors }} \text { for } i \neq j \in I \\
& \left.r_{i}^{4}=1, \text { for } i \in J\right\rangle .
\end{aligned}
$$

Proposition 18.9. (a) For all $i^{\sigma}, j^{\sigma}$ in the same connected component of $\Pi^{\mathrm{adm}}$ one has $r_{i}^{4}=r_{j}^{4}$ in $W^{\operatorname{spin}}(\Pi)$.

(b) One has $W^{\operatorname{spin}}(\Pi)=W^{\operatorname{spin}}\left(\Pi, \kappa_{\max }\right)$.

Proof. Assertion (a) is immediate from Lemma 18.6(b).

By Remark 16.5, the maximal admissible colouring $\kappa_{\max }$ is the sum of all elementary admissible colourings. By Remark 16.3 the only obstruction to being able to assign the value 2 to a vertex $k^{\sigma}$ of $\Pi$ is the existence of a vertex $i^{\sigma}$ in the same connected component of $\Pi^{\mathrm{adm}}$ as $k^{\sigma}$ such that there is a vertex $j^{\sigma}$ with $n(i, j)=0$ and $n(j, i)=1$. This implies $r_{k}^{4}=r_{i}^{4}=1$ by assertion (a) and Lemma 18.6(c).

We conclude that for all $i$ with $\kappa_{\max }\left(i^{\sigma}\right)=1$ one has $r_{i}^{4}=1$ in $W^{\text {spin }}(\Pi)$. Assertion (b) follows.

Definition 18.10. Let $D^{\operatorname{ext}}(\Pi):=\left\langle t_{i}^{2} \mid i \in I\right\rangle \leq W^{\operatorname{ext}}(\Pi)$ and $D^{\operatorname{spin}}(\Pi):=\left\langle r_{i}^{2} \mid i \in I\right\rangle \leq W^{\operatorname{spin}}(\Pi)$.

In case $\Pi$ is the Dynkin diagram of type $E_{10}$, the group $D^{\text {spin }}(\Pi)$ is isomorphic to the groups $\mathcal{D}^{v s}$ and $\mathcal{D}^{s}$ from [DH09, Propositions 1 and 2].

The similarities of the presentations of $W^{\operatorname{spin}}(\Pi), W^{\operatorname{ext}}(\Pi), W(\Pi)$ immediately yield the following:

Observation 18.11. The following hold:

(a) There is a unique epimorphism $W^{\mathrm{spin}}(\Pi) \rightarrow W^{\mathrm{ext}}(\Pi)$ mapping $r_{i}$ to $t_{i}$ and with kernel $Z$.

(b) There is a unique epimorphism $W^{\text {spin }}(\Pi) \rightarrow W(\Pi)$ mapping $r_{i}$ to $s_{i}$ and with kernel $D^{\text {spin }}$.

(c) There is a unique epimorphism $W^{\mathrm{ext}}(\Pi) \rightarrow W(\Pi)$ mapping $t_{i}$ to $s_{i}$ and with kernel $D^{\mathrm{ext}}$.

Moreover, from the literature one can extract: 
Proposition 18.12. The following hold:

(a) There is a unique isomorphism $W^{\mathrm{ext}}(\Pi) \rightarrow \widetilde{W}(\Pi)$ mapping $t_{i}$ to $\widetilde{s}_{i}$.

(b) There is a unique epimorphism $\widetilde{W}(\Pi) \rightarrow W(\Pi)$ mapping $\widetilde{s}_{i}$ to $s_{i}$.

(c) $D^{\text {ext }} \cong \operatorname{ker}(\widetilde{W}(\Pi) \rightarrow W(\Pi))$ is an elementary abelian group of order $2^{|I|}$.

Proof. (a) This follows from [KP85, Corollary 2.4] and its proof.

(b) This follows from KP85, Corollary 2.3] or, alternatively, by combining (a) with Observation 18.11.

(c) This follows from [KP85, Lemma 2.2(a) and Corollary 2.3].

Remark 18.13. Let $n \geq 2$ and let $e_{1}, \ldots, e_{n}$ be the standard basis of $\mathbb{R}^{n}$. In the following, we frequently use variations of the following basic computations in $\mathrm{Cl}(n)$ (cf. Section 6):

$$
\begin{gathered}
\left(e_{1} e_{2}\right)^{2}=-1, \quad\left(\frac{1}{\sqrt{2}}\left(1+e_{1} e_{2}\right)\right)^{2}=\frac{1}{2}\left(1+2 e_{1} e_{2}+\left(e_{1} e_{2}\right)^{2}\right)=e_{1} e_{2}, \\
\left(\frac{1}{\sqrt{2}}\left(1+e_{1} e_{2}\right)\right)^{-1}=\frac{1}{\sqrt{2}}\left(1+e_{1} e_{2}\right) \cdot\left(e_{1} e_{2}\right)^{-1}=\frac{1}{\sqrt{2}}\left(1-e_{1} e_{2}\right) .
\end{gathered}
$$

Moreover, one has

$$
\frac{1}{\sqrt{2}}\left(1+e_{1} e_{2}\right) \frac{1}{\sqrt{2}}\left(1+e_{2} e_{3}\right)+\frac{1}{\sqrt{2}}\left(1+e_{2} e_{3}\right) \frac{1}{\sqrt{2}}\left(1+e_{1} e_{2}\right)=1+e_{1} e_{2}+1+e_{2} e_{3}-1 .
$$

Proposition 18.14. For each admissible colouring $\kappa$ there is a unique epimorphism $f: W^{\operatorname{spin}}(\Pi) \rightarrow$ $\widehat{W}(\Pi, \kappa)$ mapping $r_{i}$ to $\widehat{s}_{i}$ for $i \in I$, which factors through $W^{\operatorname{spin}}(\Pi, \kappa)$ :

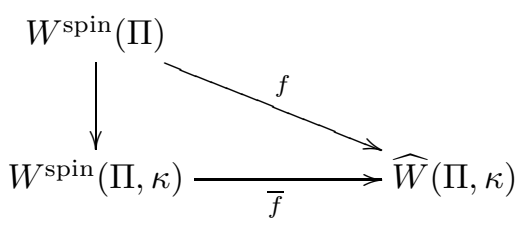

Proof. The $r_{i}$ generate $W^{\text {spin }}$, so if $f$ exists, it is unique. Moreover, the $\widehat{s}_{i}$ generate $\widehat{W}$, so if $f$ exists, it is also surjective.

It remains to show that $f$ maps every relator of $W^{\text {spin }}$ to $1_{\widehat{W}}$. Since all relators of $W^{\text {spin }}$ involve at most two generators it suffices to consider the local situation in all rank 2 subgroups, i.e., to look at all pairs $i \neq j \in I$ and verify that $r_{i}, r_{j}$ and the relations between them are mapped correctly.

For $i \neq j \in I$ define

$$
x_{j}:= \begin{cases}\tilde{\phi}_{i j}^{j}\left(S\left(\frac{\pi}{4}\right)\right), & \text { if } j \in I \backslash J, \\ \tilde{\phi}_{i j}^{j}\left(S\left(\frac{\pi}{2}\right)\right), & \text { if } j \in J .\end{cases}
$$

Thus for all $j \in I$ one has $\widehat{s}_{j}=\tilde{\psi}_{i j}\left(x_{j}\right)$ according to Definition 18.3. Certainly, the relations R1 (and R4) hold for the $\widehat{s}_{j}$; one has

$$
x_{j}^{8}=\left\{\begin{array}{cc}
\tilde{\phi}_{i j}^{j}(S(2 \pi)), & \text { if } j \in I \backslash J, \\
\tilde{\phi}_{i j}^{j}(S(4 \pi)), & \text { if } j \in J
\end{array}\right\}=1 .
$$

Define $v:=v_{i j}$, i.e., $\{i, j\}^{\sigma} \in E_{v}(\Pi)$, and $k:=k_{i j}$. We check the relations for each of the possible values of $(v, k)$ in Notation 16.8

$\mathbf{k}=\mathbf{1}$ : This case is well-known, see e.g. Proposition $18.12(\mathrm{a})$.

$\mathbf{v}=\mathbf{0}, \mathbf{k}>\mathbf{1}$ : We have $G_{i j}=\operatorname{Spin}(2) \times \operatorname{Spin}(2)$. Recall Notation 8.1. Then

$$
\begin{aligned}
x_{i} & =\tilde{\iota}_{1}\left(S\left(\frac{\pi}{4}\right)\right), \\
x_{j} & =\tilde{\iota}_{2}\left(S\left(\frac{\pi}{4}\right)\right) .
\end{aligned}
$$

Relation R2

$$
\begin{aligned}
& x_{i}^{-1} x_{j}^{2} x_{i}=x_{j}^{2}=x_{j}^{2} x_{i}^{2 n(i, j)}, \\
& x_{j}^{-1} x_{i}^{2} x_{j}=x_{i}^{2}=x_{i}^{2} x_{j}^{2 n(j, i)} .
\end{aligned}
$$

Relation R3

$$
x_{j} x_{i}=x_{i} x_{j} .
$$


$(\mathbf{v}, \mathbf{k})=(\mathbf{1}, \mathbf{2}):$ We have $G_{i j}=\operatorname{Spin}(3)$. Recall Remark6.13, Then

$$
\begin{aligned}
& x_{i}=\tilde{\varepsilon}_{23}\left(S\left(\frac{\pi}{4}\right)\right)=\frac{1}{\sqrt{2}}\left(1+e_{2} e_{3}\right), \\
& x_{j}=\tilde{\varepsilon}_{12}\left(S\left(\frac{\pi}{4}\right)\right)=\frac{1}{\sqrt{2}}\left(1+e_{1} e_{2}\right) .
\end{aligned}
$$

Relation R2.

$$
\begin{aligned}
x_{i}^{-1} x_{j}^{2} x_{i} & =\frac{1}{\sqrt{2}}\left(1-e_{2} e_{3}\right) \cdot e_{1} e_{2} \cdot \frac{1}{\sqrt{2}}\left(1+e_{2} e_{3}\right) \\
& =\frac{1}{2}\left(e_{1} e_{2}+e_{1} e_{2} e_{2} e_{3}-e_{2} e_{3} e_{1} e_{2}-e_{2} e_{3} e_{1} e_{2} e_{2} e_{3}\right) \\
& =\frac{1}{2}\left(e_{1} e_{2}-e_{1} e_{3}-e_{1} e_{3}-e_{1} e_{2}\right) \\
& =-e_{1} e_{3}=e_{1} e_{2} e_{2} e_{3}=x_{j}^{2} x_{i}^{2}=x_{j}^{2} x_{i}^{2 n(i, j)}, \\
x_{j}^{-1} x_{i}^{2} x_{j} & =x_{i}^{2} x_{j}^{2}=x_{i}^{2} x_{j}^{2 n(j, i)} .
\end{aligned}
$$

Relation R3.

$$
\begin{aligned}
x_{j} x_{i} x_{j} & =x_{j} \frac{1}{\sqrt{2}}\left(1+e_{2} e_{3}\right) x_{j}=\frac{1}{\sqrt{2}}\left(x_{j}^{2}+x_{j} x_{i}^{2} x_{j}\right) \\
& =\frac{1}{\sqrt{2}}\left(x_{j}^{2}+x_{j}\left(x_{i}^{2} x_{j}^{2}\right) x_{j}^{-1}\right) \\
& =\frac{1}{\sqrt{2}}\left(x_{j}^{2}+x_{j}\left(x_{j}^{-1} x_{i}^{2} x_{j}\right) x_{j}^{-1}\right) \\
& =\frac{1}{\sqrt{2}}\left(x_{j}^{2}+x_{i}^{2}\right) .
\end{aligned}
$$

By symmetry, we conclude $x_{j} x_{i} x_{j}=x_{i} x_{j} x_{i}$.

$(\mathbf{v}, \mathbf{k})=(\mathbf{2}, \mathbf{1 . 5}), \mathbf{i}^{\sigma} \rightarrow \mathbf{j}^{\sigma}:$ We have $G_{i j}=\mathrm{SO}(2) \times \mathrm{SU}_{2}(\mathbb{C})$ and $j \in J$. Then

$$
\begin{aligned}
& x_{i}=\tilde{\zeta}_{p}\left(S\left(\frac{\pi}{4}\right)\right)=\frac{1}{\sqrt{2}}\left(\left(\begin{array}{cc}
1 & 1 \\
-1 & 1
\end{array}\right),\left(\begin{array}{cccc}
1 & -1 & \\
1 & 1 & & \\
& & 1 & 1
\end{array}\right)\right) \text {, } \\
& \text { (cf. Notation 14.8) }
\end{aligned}
$$

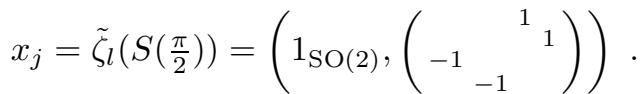

Relation R2.

$$
\begin{aligned}
& x_{i}^{-1} x_{j}^{2} x_{i}=\left(1_{\mathrm{SO}(2)},-1_{\mathrm{SU}_{2}(\mathbb{C})}\right)=x_{j}^{2}=x_{j}^{2} x_{i}^{2 \cdot 0}=x_{j}^{2} x_{i}^{2 n(i, j)} \\
& x_{j}^{-1} x_{i}^{2} x_{j}=\left(\left({ }_{-1}{ }^{1}\right),\left(\begin{array}{cc}
{ }_{-1}{ }_{-1} & \\
1
\end{array}\right)\right)=x_{i}^{2} x_{j}^{2}=x_{i}^{2} x_{j}^{2 \cdot 1}=x_{i}^{2} x_{j}^{2 n(j, i)} .
\end{aligned}
$$

Relation R3.

$$
x_{j} x_{i} x_{j} x_{i}=\left(x_{j} x_{i}\right)^{2}=\left(\left({ }_{-1}^{1}\right),-1_{\mathrm{SU}_{2}(\mathbb{C})}\right)=\left(x_{i} x_{j}\right)^{2}=x_{i} x_{j} x_{i} x_{j} . .
$$

$(\mathbf{v}, \mathbf{k})=(\mathbf{3}, \mathbf{2}), \mathbf{i}^{\sigma} \rightarrow \mathbf{j}^{\sigma}:$ We have $G_{i j}=\operatorname{Spin}(4)$. Recall Notation 13.9, Then

$$
\begin{aligned}
x_{i} & =\tilde{\eta}_{p}\left(S\left(\frac{\pi}{4}\right)\right)=\tilde{\varepsilon}_{34}\left(S\left(\frac{\pi}{4}\right)\right)=\frac{1}{\sqrt{2}}\left(1+e_{3} e_{4}\right) \\
x_{j} & =\tilde{\eta}_{l}\left(S\left(\frac{\pi}{4}\right)\right)=\tilde{\varepsilon}_{14}\left(S\left(2 \cdot \frac{\pi}{4}\right)\right) \cdot \tilde{\varepsilon}_{23}\left(S\left(-\frac{\pi}{4}\right)\right)=e_{1} e_{4} \cdot \frac{1}{\sqrt{2}}\left(1-e_{2} e_{3}\right) \\
& =\frac{1}{\sqrt{2}}\left(e_{1} e_{4}+e_{1} e_{2} e_{3} e_{4}\right) .
\end{aligned}
$$

Relation R2.

$$
\begin{aligned}
& x_{i}^{-1} x_{j}^{2} x_{i}=e_{2} e_{4}=x_{j}^{2} x_{i}^{2}=x_{j}^{2} x_{i}^{2 \cdot 1}=x_{i}^{2} x_{j}^{2 n(i, j)}, \\
& x_{j}^{-1} x_{i}^{2} x_{j}=-e_{2} e_{4}=x_{i}^{2} x_{j}^{2}=x_{j}^{2} x_{i}^{2 \cdot 1}=x_{j}^{2} x_{i}^{2 n(j, i)} .
\end{aligned}
$$

Relation [R3 $x_{j} x_{i} x_{j} x_{i} x_{j} x_{i}=\left(x_{j} x_{i}\right)^{3}=-e_{1} e_{2} e_{3} e_{4}=\left(x_{i} x_{j}\right)^{3}=x_{i} x_{j} x_{i} x_{j} x_{i} x_{j}$. $\mathbf{v}=\infty, \mathbf{k}>\mathbf{1}, \mathbf{i}^{\sigma} \rightarrow \mathbf{j}^{\sigma}:$ We have $G_{i j}=\widetilde{K}_{1} \widetilde{U} * \widetilde{U}_{2} \widetilde{U}$. Recall Definition 15.8. Then

$$
\begin{aligned}
& x_{i}=\tilde{\theta}_{1}\left(S\left(\frac{\pi}{4}\right)\right), \\
& x_{j}=\left\{\begin{array}{ll}
\tilde{\theta}_{2}\left(S\left(\frac{\pi}{2}\right)\right), & \text { if } j \in J \\
\tilde{\theta}_{2}\left(S\left(\frac{\pi}{4}\right)\right), & \text { if } j \in I \backslash J .
\end{array} \quad(\Longleftrightarrow n(i, j)=1, n(j, i)=0),\right.
\end{aligned}
$$

Relation R2, As discussed in Remark 18.5 one has

$$
\begin{aligned}
& x_{i}^{-1} x_{j}^{2} x_{i}=x_{j}^{2} x_{i}^{2 n(j, i)} \Longleftrightarrow x_{j}^{-2} x_{i}^{-1} x_{j}^{2}=x_{i}^{2 n(j, i)} x_{i}^{-1}, \\
& x_{j}^{-1} x_{i}^{2} x_{j}=x_{i}^{2} x_{j}^{2 n(i, j)} \Longleftrightarrow x_{i}^{-2} x_{j}^{-1} x_{i}^{2}=x_{j}^{2 n(i, j)} x_{j}^{-1},
\end{aligned}
$$


Since $x_{i} \in \widetilde{K}_{1}, x_{j} \in \widetilde{K}_{2}, x_{i}^{2}=\tilde{t}_{1}, x_{j}^{2}=\tilde{t}_{2}$ (cf. Remark 15.9), the respective equalities on the right hand sides hold by identity (2) in Proposition 15.10.

Theorem 18.15. The group homomorphism

$$
\bar{f}: W^{\operatorname{spin}}(\Pi, \kappa) \rightarrow \widehat{W}(\Pi, \kappa)
$$

mapping $r_{i} \mapsto \widehat{s}_{i}$ is an isomorphism. In particular

$$
W^{\operatorname{spin}}(\Pi) \cong \widehat{W}\left(\Pi, \kappa_{\max }\right)
$$

Proof. We already established that $\bar{f}$ is an epimorphism. It remains to prove that it is injective.

Let $\rho: \operatorname{Spin}(\Pi, \kappa) \rightarrow K(\Pi)$ be the central extension from Theorem 17.1. Then, using Lemmas 10.4. 13.11, 14.10 and Proposition 15.10, it follows that

$$
\begin{aligned}
\rho\left(\widehat{s}_{i}\right) & = \begin{cases}\left(\rho \circ \tilde{\psi}_{i j} \circ \tilde{\phi}_{i j}^{i} \circ S\right)\left(\frac{\pi}{4}\right) & \text { if } i \notin J \\
\left(\rho \circ \tilde{\psi}_{i j} \circ \tilde{\phi}_{i j}^{i} \circ \mathrm{sq} \circ S\right)\left(\frac{\pi}{4}\right) & \text { if } i \in J\end{cases} \\
& = \begin{cases}\left(\psi_{i j} \circ \rho^{\left(v_{i j}\right)} \circ \tilde{\phi}_{i j}^{i} \circ S\right)\left(\frac{\pi}{4}\right) & \text { if } i \notin J \\
\left(\psi_{i j} \circ \rho^{\left(v_{i j}\right)} \circ \tilde{\phi}_{i j}^{i} \circ \mathrm{sq} \circ S\right)\left(\frac{\pi}{4}\right) & \text { if } i \in J\end{cases} \\
& =\left(\psi_{i j} \circ \phi_{i j}^{i} \circ \rho_{2} \circ S\right)\left(\frac{\pi}{4}\right)=\left(\psi_{i j} \circ \phi_{i j}^{i} \circ D\right)\left(\frac{\pi}{2}\right)=\tilde{s}_{i} .
\end{aligned}
$$

Since $\operatorname{ker} \rho=\left\langle\left(\widehat{s}_{i}\right)^{4} \mid i \in I\right\rangle \subseteq \widehat{W}(\Pi, \kappa)$, the restriction of $\rho$ to an epimorphism $\widehat{W}(\Pi, \kappa) \rightarrow \widetilde{W}(\Pi)$ is a $2^{c(\Pi, \kappa)}$-fold central extension by Theorem 17.1. Consider the following diagram:

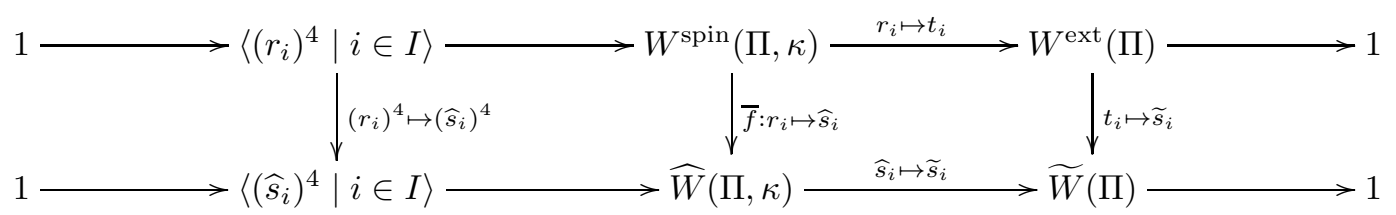

The upper row of this diagram is exact by Lemma 18.11(a), the lower row by the above discussion. It is not hard to verify that the diagram is also commutative. By Consequence 18.7 the group $\left\langle\left(r_{i}\right)^{4}\right|$ $i \in I\rangle$ is abelian and by Proposition 18.9 it has at most $2^{c(\Pi, \kappa)}$ elements. Since $\left|\left\langle\left(\widehat{s}_{i}\right)^{4} \mid i \in I\right\rangle\right|=$ $2^{c(\Pi, \kappa)}$ by the above discussion, the left-hand arrow is an isomorphism. The right-hand arrow is an isomorphism by Proposition 18.12 (a). Thus $\bar{f}$ is also an isomorphism. The final statement follows from Proposition 18.9(b).

We have proved Theorem $\mathrm{B}$ from the introduction.

\section{Finite-dimensional Compact Lie group quotients of Spin( $\Pi)$ And $K(\Pi)$}

In this section we prove that for each simply laced diagram $\Pi$ the group $\operatorname{Spin}(\Pi)$ admits an epimorphism onto a non-trivial compact Lie group afforded by the representation constructed in Theorem [11.14. By virtue of Theorem 17.10 such an epimorphism then in fact exists for any diagram $\Pi$ and any admissible colouring $\kappa$.

Theorem 19.1. Let $\Pi$ be a simply laced diagram. Then the target of the (continuous) group epimorphism $\Xi: \operatorname{Spin}(\Pi) \rightarrow X$ from Remark11.15 is a non-trivial compact Lie group, as is the target of the induced (continuous) group epimorphism $\bar{\Xi}: K(\Pi) \rightarrow X /\langle\Xi(Z)\rangle$.

Proof. By [HKL13, Theorem 4.11] the image of the generalized spin representation $\mu: \mathfrak{k} \rightarrow \operatorname{End}\left(\mathbb{C}^{s}\right)$ from Theorem 11.14 is compact. Hence the claim for $\Xi$, and by the homomorphism theorem for groups also for $\bar{\Xi}$, follows.

Corollary 19.2 (cf. [DH09, Lemma 2, p. 49]). Let П be a simply laced diagram. Then the image $\Xi(\widehat{W}(\Pi))$ is finite. 
Proof. As in the proof of [DH09, Lemma 2, p. 49] the key observation is that $\Xi(\widehat{W}(\Pi)) \leq X$ is a discrete subgroup of the compact group X. Consider the commutative diagram obtained from Theorem 11.14

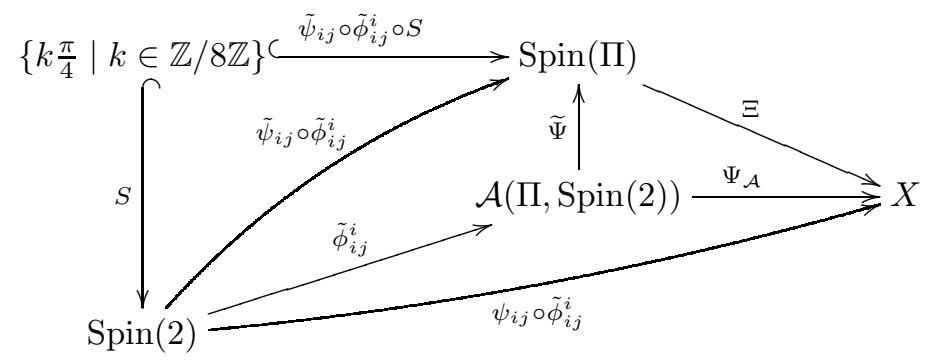

where

$$
\widetilde{\Psi}=\left\{\tilde{\psi}_{i j}: \widetilde{G}_{i j} \rightarrow \operatorname{Spin}(\Pi)\right\}:=\tau_{\operatorname{Spin}(\Pi)}=\left\{\tau_{i j}: \widetilde{G}_{i j} \rightarrow \operatorname{Spin}(\Pi)\right\}
$$

is the canonical universal enveloping morphism of the amalgam $\mathcal{A}(\Pi, \operatorname{Spin}(2))=\left\{\widetilde{G}_{i j}, \tilde{\phi}_{i j}^{i} \mid i \neq j \in I\right\}$. Then

where $\widehat{s}_{i}:=\tilde{\psi}_{i j}\left(\tilde{\phi}_{i j}^{i}\left(S\left(\frac{\pi}{4}\right)\right)\right)$ and

$$
\widehat{W}(\Pi)=\left\langle\widehat{s}_{i} \mid i \in I\right\rangle
$$

$$
\Xi(\widehat{W}(\Pi))=\left\langle\psi_{i j}\left(\tilde{\phi}_{i j}^{i}\left(S\left(\frac{\pi}{4}\right)\right)\right) \mid i \in I\right\rangle=\left\langle\cos \left(\frac{\pi}{4}\right)+\sin \left(\frac{\pi}{4}\right) X_{i} \mid i \in I\right\rangle .
$$

For $R_{i}:=\cos \left(\frac{\pi}{4}\right)+\sin \left(\frac{\pi}{4}\right) X_{i}=\frac{\sqrt{2}}{2}+\frac{\sqrt{2}}{2} X_{i}$ the commutator relations

$$
X_{i} X_{j}= \begin{cases}-X_{j} X_{i}, & \text { if }\{i, j\}^{\sigma} \in E(\Pi), \\ X_{j} X_{i}, & \text { if }\{i, j\}^{\sigma} \notin E(\Pi)\end{cases}
$$

established in [HKL13, Remark 4.5] (cf. the proof of Theorem 11.14) imply that

$$
R_{i} R_{j}= \begin{cases}-R_{j} R_{i}+\sqrt{2} R_{i}+\sqrt{2} R_{j}-1, & \text { if }\{i, j\}^{\sigma} \in E(\Pi), \\ R_{j} R_{i}, & \text { if }\{i, j\}^{\sigma} \notin E(\Pi),\end{cases}
$$

as in Remark 18.13] or in the proof of [DH09, Lemma 2, p. 49]; moreover, $X_{i}^{2}=-$ id implies $R_{i}^{2}=\sqrt{2} R_{i}-1$.

We conclude that any product of the above generators of $\Xi(\widehat{W}(\Pi))$ can be written as a polynomial in the $R_{i}, i \in I$, such that each $R_{i}$ appears at most linearly in each polynomial, with coefficients in $\mathbb{Z}[\sqrt{2}]$. Hence $\Xi(\widehat{W}(\Pi))$ is a discrete set. As it is a subgroup of the compact group $X$, it has to be finite.

Not much is known about the kernels of the maps $\Xi$ and $\Xi$. However, Pierre-Emmanuel Caprace pointed out to us that these kernels generally cannot be abstractly simple. The argument relies on the concept of acylindrical hyperbolicity (see Osi13, also [CH14]).

Corollary 19.3. Let $\Pi$ be an irreducible non-spherical, non-affine simply laced Dynkin diagram. Then the kernels of the generalized spin representations $\Xi: \operatorname{Spin}(\Pi) \rightarrow X$ and $\Xi: K(\Pi) \rightarrow X /\langle\Xi(Z)\rangle$ are acylindrically hyperbolic and, in particular, not abstractly simple.

Proof. Spin( $\Pi)$ and $K(\Pi)$ naturally act on each half of the twin building of the associated Kac-Moody group $G(\Pi)$ and, thus, so do $\operatorname{ker}(\Xi)$ and $\operatorname{ker}(\bar{\Xi})$. The stabiliser in $K(\Pi)$ of the fundamental chamber consists of the elements of the standard torus of $G(\Pi)$ that square to the identity, the stabiliser in $\operatorname{Spin}(\Pi)$ is a double extension of this group. In particular, chamber stabilisers in $K(\Pi)$ and in $\operatorname{Spin}(\Pi)$ are finite and, hence, so are chamber stabilisers in $\operatorname{ker}(\bar{\Xi})$ and $\operatorname{ker}(\Xi)$. By Corollary 19.2 the intersection $\operatorname{ker}(\bar{\Xi}) \cap \widetilde{W}(\Pi)$ has finite index in $\widetilde{W}(\Pi)$ and the intersection $\operatorname{ker}(\Xi) \cap \widehat{W}(\Pi)$ has finite index in $\widehat{W}(\Pi)$ (see Section 18 for definitions). Hence [CH14, Theorem 1.4] applies and $\operatorname{ker}(\Xi)$ and $\operatorname{ker}(\bar{\Xi})$ are acylindrically hyperbolic. Therefore by [DGO11, Theorem 8.5] both groups contain a non-trivial proper free normal subgroup.

Corollary 19.4. Let $\Pi$ be a Dynkin diagram with admissible colouring $\kappa$. Then the target of the group homomorphism $\operatorname{Spin}(\Pi, \kappa) \stackrel{\Xi_{\mathrm{Spin}(\Pi, \kappa)}}{\longrightarrow} \operatorname{Spin}\left(\Pi^{\mathrm{dl} \kappa}, \kappa\right) \stackrel{\left.\Omega_{\mathrm{Spin}(\Pi \mathrm{dl} l \kappa}, \kappa\right)}{\longrightarrow} \operatorname{Spin}\left(\left(\Pi^{\mathrm{dl}}\right)^{\mathrm{un}}, \kappa^{\mathrm{un}}\right) \stackrel{\Xi}{\longrightarrow} X$ is a non-trivial compact Lie group, as is the target of the induced group epimorphism $K(\Pi) \stackrel{\Xi_{K(\Pi)}}{\longrightarrow} K\left(\left(\Pi^{\mathrm{dl} \kappa}\right) \stackrel{\Omega_{K\left(\Pi_{\mathrm{dl}} \kappa\right)}}{\longrightarrow}\right.$ $\left.K\left(\Pi^{\mathrm{dl}}\right)^{\mathrm{un}}\right) \stackrel{\Xi}{\Xi} X /\langle\Xi(Z)\rangle$. 
Proof. It suffices to observe that $\operatorname{Spin}\left(\Pi^{\mathrm{d} 1 \kappa}, \kappa\right) \stackrel{\Omega_{\mathrm{Spin}\left(\Pi_{\mathrm{d}} \kappa, \kappa\right)}}{\longrightarrow} \operatorname{Spin}\left(\left(\Pi^{\mathrm{dl}}\right)^{\mathrm{un}}, \kappa^{\mathrm{un}}\right)$ and $K\left(\Pi^{\mathrm{dl} \kappa}\right) \stackrel{\Omega_{K\left(\Pi^{\mathrm{dl} \kappa}\right)}}{\longrightarrow} K\left(\left(\Pi^{\mathrm{dl}}\right)^{\mathrm{un}}\right)$ have closed images with respect to the Kac-Peterson topology, as they can be realized as fixed points of continuous involutions.

Remark 19.5. For $\Pi=E_{n}$ for some $n \in \mathbb{N}$, the isomorphism type of $X$ can be extracted from the Cartan-Bott periodicity described in [HK14, Theorem A].

\section{REFERENCES}

[BC13] Francis Buekenhout and Arjeh Cohen. Diagram Geometry. Springer, Berlin, 2013.

[BH99] Martin Bridson and André Haefliger. Metric spaces of non-positive curvature. Springer, Berlin, 1999.

[Bor84] Mikhail Borovoi. Generators and relations in compact Lie groups. Funktsional. Anal. i. Prilozhen, 18:57-58, 1984 .

[Bou02] Nicolas Bourbaki. Lie groups and Lie algebras. Chapters 4-6. Springer-Verlag, Berlin, 2002.

[Car89] Roger W. Carter. Simple groups of Lie type. John Wiley \& Sons, Inc., New York, 1989.

[CH14] Pierre-Emmanuel Caprace and David Hume. Orthogonal forms of Kac-Moody groups are acylindrically hyperbolic. arXiv: 1408.6117v2, 2014.

[Coh95] Arjeh Cohen. Point-line spaces related to buildings. In Handbook of incidence geometry, pages 647-737. NorthHolland, Amsterdam, 1995.

[DBHP06] Sophie De Buyl, Marc Henneaux, and Louis Paulot. Extended E $E_{8}$ invariance of 11-dimensional supergravity. J. High Energy Phys., (2):056, 11 pp. (electronic), 2006.

[DGO11] Francois Dahmani, Vincent Guirardel, and Denis Osin. Hyperbolically embedded subgroups and rotating families in groups acting on hyperbolic spaces. arXiv:1111.7048, 2011.

[DH09] Thibault Damour and Christian Hillmann. Fermionic Kac-Moody billards and supergravity. J. High Energy Phys., 2009, 2009. article 100.

[DKN06] Thibault Damour, Axel Kleinschmidt, and Hermann Nicolai. Hidden symmetries and the fermionic sector of eleven-dimensional supergravity. Phys. Lett. B, 634(2-3):319-324, 2006.

[DMGH09] Tom De Medts, Ralf Gramlich, and Max Horn. Iwasawa decompositions of split Kac-Moody groups. J. Lie Theory, 19:311-337, 2009.

[Gal14] Jean Gallier. Clifford Algebras, Clifford Groups, and a Generalization of the Quaternions: The Pin and Spin Groups. arXiv:0805.0311v3, 2014.

[GGH10] Helge Glöckner, Ralf Gramlich, and Tobias Hartnick. Final group topologies, Kac-Moody groups and Pontryagin duality. Israel J. Math., 177:49-101, 2010.

[Gol80] David M. Goldschmidt. Automorphisms of trivalent graphs. Ann. of Math., 111:377-406, 1980.

[Gra98] Ralf Gramlich. Homomorphisms of generalized polygons. Master's thesis, Universität Würzburg, 1998.

[Gra06] Ralf Gramlich. Defining amalgams of compact Lie groups. J. Lie Theory, 16:1-18, 2006.

[GV00] Ralf Gramlich and Hendrik Van Maldeghem. Epimorphisms of generalized polygons. I. Geometrical characterizations. Codes Cryptogr., 21:99-111, 2000.

[Hat02] Allen Hatcher. Algebraic topology. Cambridge University Press, Cambridge, 2002.

[HK14] Max Horn and Ralf Köhl. Cartan-Bott periodicity for the split real $E_{n}$ series. Appendix to Generalized spin representations by Guntram Hainke, Ralf Köhl, Paul Levy. arXiv: 1403.4463, 2014.

[HKL13] Guntram Hainke, Ralf Köhl, and Paul Levy. Generalized spin representations. With an appendix by Ralf Köhl and Max Horn: Cartan-Bott periodicity for the real $E_{n}$ series. arXiv:1110.5576, 2013.

[HKM13] Tobias Hartnick, Ralf Köhl, and Andreas Mars. On topological twin buildings and topological split Kac-Moody groups. Innov. Incidence Geom., 13:1-71, 2013.

[HM98] Karl Hofmann and Sydney Morris. The structure of compact groups. De Gruyter, Berlin, 1998.

[HO89] Alexander J. Hahn and O. Timothy O'Meara. The classical groups and K-theory. Springer, Berlin, 1989.

[IS02] Alexander Ivanov and Sergey Shpectorov. Geometry of sporadic groups. II. Cambridge University Press, Cambridge, 2002.

[Kac90] Victor Kac. Infinite dimensional Lie algebras. Cambridge University Press, 1990.

[KP83] Victor Kac and Dale Peterson. Regular functions on certain infinite-dimensional groups. In Arithmetic and geometry, Vol. II, pages 141-166. Birkhäuser Boston, Boston, MA, 1983.

[KP85] Victor Kac and Dale Peterson. Defining relations of certain infinite-dimensional groups. Astérisque, pages 165208, 1985

[KS70] A. Karrass and D. Solitar. The subgroups of a free product of two groups with an amalgamated subgroup. Trans. Amer. Math. Soc., 150:227-255, 1970.

[LM89] H. Blain Lawson and Marie-Louise Michelsohn. Spin geometry. Princeton, New Jersey, 1989.

[Mar13] Timothée Marquis. Topological Kac-Moody groups and their subgroups. PhD thesis, Université Catholique de Louvain, 2013.

[Mei13] Eckhard Meinrenken. Clifford algebras and Lie theory. Springer, Berlin, 2013.

[Osi13] Denis Osin. Acylindrically hyperbolic groups. arXiv: 1304.1246, 2013.

[Rém02] Bertrand Rémy. Groupes de Kac-Moody déployés et presque déployés. Société Mathématique de France, Paris, 2002.

[RT87] Mark Ronan and Jacques Tits. Building buildings. Math. Ann., 278:291-306, 1987.

[RT94] Mark Ronan and Jacques Tits. Twin trees. I. Invent. Math., 116:463-479, 1994.

[SBG+ ${ }^{+95] ~ H e l m u t ~ S a l z m a n n, ~ D i e t e r ~ B e t t e n, ~ T h e o ~ G r u n d h o ̈ f e r, ~ H e r m a n n ~ H a ̈ h l, ~ R a i n e r ~ L o ̈ w e n, ~ a n d ~ M a r k u s ~ S t r o p p e l . ~}$ Compact projective planes. De Gruyter, Berlin, 1995.

[Ser03] Jean-Pierre Serre. Trees. Springer, Berlin, 2003.

[Tit66] Jacques Tits. Normalisateurs de tores. I. Groupes de Coxeter étendus. J. Algebra, 4:96-116, 1966. 
[Tit74] Jacques Tits. Buildings of spherical type and finite BN-pairs. Springer, Berlin, 1974.

[Tit81] Jacques Tits. A local approach to buildings. In The geometric vein, pages 519-547. Springer, New York, 1981.

[Tit86] Jacques Tits. Ensembles ordonnés, immeubles et sommes amalgamées. Bull. Soc. Math. Belg. Sér. A, 38:367$387,1986$.

[Tit87] Jacques Tits. Uniqueness and presentation of Kac-Moody groups over fields. J. Algebra, 105:542-573, 1987.

[VM98] Hendrik Van Maldeghem. Generalized polygons, volume 93 of Monographs in Mathematics. Birkhäuser Verlag, Basel, 1998.

D.G.: University of Birmingham, School of Mathematics, Edgbaston, Birmingham, B15 2TT, United Kingdom

M.H., R.K., S.W.: JLU Giessen, Mathematisches Institut, Arndtstrasse 2, 35392 Giessen, Germany

E-mail address: max.horn@math.uni-giessen.de

E-mail address: ralf.koehl@math.uni-giessen.de 